\title{
New light on the formation and evolution of M 31 and its globular cluster system ${ }^{\star}$
}

\author{
T. H. Puzia ${ }^{1, \star \star}$, K. M. Perrett ${ }^{2, \star \star \star}$, and T. J. Bridges ${ }^{3}$ \\ 1 Space Telescope Science Institute, 3700 San Martin Drive, Baltimore, MD 21218, USA \\ e-mail: tpuzia@stsci.edu \\ 2 Department of Astronomy \& Astrophysics, University of Toronto, Toronto, ON, M5S 3H8, Canada \\ e-mail: perrett@astro.utoronto.ca \\ 3 Department of Physics, Queen's University, Kingston, ON, K7L 3N6, Canada \\ e-mail: tjb@astro.queensu.ca
}

Received 6 August 2004 / Accepted 13 January 2005

\begin{abstract}
We present spectroscopic ages, metallicities, and $[\alpha / \mathrm{Fe}]$ ratios for 70 globular clusters in M 31 that were derived from Lick line-index measurements. A new interpolation technique of age-metallicity and $\alpha / \mathrm{Fe}$-diagnostic grids is used to account for changes in index strength as a response to abundance-ratio variations, in particular for all of the Balmer-line Lick indices. In addition to a population of old ( $>10 \mathrm{Gyr})$ globular clusters with a wide range of metallicities, from about -2.0 dex to solar values, we find evidence for a population of intermediate-age globular clusters with ages between $\sim 5$ and $8 \mathrm{Gyr}$ and a mean metallicity $[\mathrm{Z} / \mathrm{H}] \approx-0.6$. We also confirm the presence of young M 31 globular clusters that were recently identified by Beasley et al. (2004, AJ, 128, 1623), which have ages $\lesssim 1$ Gyr and relatively high metallicities around -0.4 dex. The M 31 globular cluster system has a clearly super-solar mean $[\alpha / \mathrm{Fe}]=0.14 \pm 0.04$ dex. Intermediate-age and young objects show roughly solar abundance ratios. We find evidence for an age- $[\alpha / \mathrm{Fe}]$ relation in the sense that younger clusters have smaller mean $[\alpha / \mathrm{Fe}]$ ratios. From a comparison of indices, mostly sensitive to carbon and/or nitrogen abundance, with SSP model predictions for nitrogen-enhanced stellar populations, we find a dichotomy in nitrogen enhancement between young and old M 31 globular clusters. The indices of objects older than 5 Gyr are consistent with a factor of three or higher in nitrogen enhancement compared to their younger counterparts. Using kinematical data from Morrison et al. (2004, ApJ, 603, 87) we find that the globular cluster sub-population with halo kinematics is old ( $\gtrsim 9 \mathrm{Gyr}$ ), has a bimodal metallicity distribution, and super-solar $[\alpha / \mathrm{Fe}]$. Disk globular clusters have a wider range of ages, are on average more metal-rich, and have a slightly smaller mean $[\alpha / \mathrm{Fe}]$ ratio. A cross-correlation of structural parameters for M 31 globular clusters with spectroscopically derived ages, metallicities, and $[\alpha / \mathrm{Fe}]$ ratios shows a correlation between half-light/tidal radius and metallicity, which is most likely due to the correlation of half-light/tidal radius and galactocentric distance. We compare our results for M 31 globular clusters with those obtained with the same technique for globular clusters in the Milky Way, Large Magellanic Cloud, M 81, and other spiral galaxies in the Sculptor group. Finally, we compare the globular cluster systems of the two Local Group spirals, M 31 and Milky Way, with their integrated bulge light.
\end{abstract}

Key words. galaxies: star clusters - galaxies: formation - galaxies: evolution - galaxies: Local Group Galaxy: globular clusters: general - galaxies: general

\section{Introduction}

Because they can be resolved into single stars, the stellar populations in the Local Group are milestones for understanding star formation and chemical evolution histories of distant galaxies. Globular clusters host simple stellar populations that are characterized by their small dispersion in age and chemical composition, and are, compared to the mix of stellar populations in galaxies, relatively easy to understand. All theoretical predictions for observables of unresolved stellar systems are

\footnotetext{
* Appendix A is only available in electronic form at http://www . edpsciences.org

$\star \star$ ESA Fellow, Space Telescope Division of ESA.

$\star \star \star$ NSERC Post-doctoral Fellow.
}

calibrated on resolved stellar populations in Milky Way globular clusters. Since the formation of globular clusters is temporally linked with major star-formation episodes, globular cluster systems are fossil records of the past star formation and chemical evolution history of their host galaxies (Ashman \& Zepf 1998; Harris 2001). It is therefore of paramount importance to ensure that the predictions drawn from a comparison of Galactic and extragalactic globular clusters, and stellar populations in general, are not biased by any peculiarity of our neighborhood.

The richest globular cluster systems in the Local Group are hosted by the two major spiral galaxies Andromeda (M 31; NGC 224) harboring $460 \pm 70$ (Barmby \& Huchra 2001) 
and the Milky Way with $160 \pm 20$ globular clusters (Harris 2001). Photometry has shown that the globular cluster systems of M 31 and Milky Way have a similar range in metallicity and that both distributions are bimodal with peaks around $[\mathrm{Fe} / \mathrm{H}]=-1.4$ and -0.6 dex (e.g. Barmby et al. 2000; Barmby \& Huchra 2001). Recently, Perrett et al. (2002) spectroscopically confirmed this metallicity bimodality. However, M 31 hosts a larger number of metal-rich clusters, which is reflected in the higher mean metallicity of the entire globular cluster system (e.g. Huchra et al. 1991). Although the globular cluster luminosity functions are remarkably similar in both galaxies (Harris 2001), they may differ as a function of metallicity and galactocentric radius (Barmby et al. 2001). There is also a difference in spatial distribution (scale size), where the M 31 cluster system appears to be more extended than the Galactic globular cluster system (Harris 2001). Thus, despite the overall similarities of the M 31 and Milky Way globular cluster systems, there are some striking differences in the details. Recently, a revised optical/near-IR photometric catalog of 337 confirmed and 688 candidate globular clusters in M 31, that combines and homogenizes previous studies, was published by the Bologna group and will allow for more accurate future analyses (Galleti et al. 2004, and references therein).

Integrated-light spectroscopy of M 31 globular clusters was pioneered by van den Bergh (1969) who found that the M 31 globular cluster system extends to much higher metallicities than the Milky Way globular cluster system. Subsequent studies confirmed these results (e.g. Spinrad \& Schweizer 1972; Rabin 1981). A thorough study, based on spectroscopic Lick line indices of 19 M 31 and 17 Galactic globular clusters, by Burstein et al. (1984) confirmed this and showed that M 31 clusters have significantly stronger $\mathrm{H} \beta$ and $\mathrm{CN}$ absorption indices at a given metallicity. After carefully checking the influence of hot horizontal-branch stars and/or blue stragglers, and younger ages on these indices, the authors concluded that age is the most favorable, but not sufficient, explanation for the systematic differences. They speculated that their sample was dominated by relatively young disk globular clusters, which could explain the offset in $\mathrm{H} \beta$, but not the strong $\mathrm{CN}$ indices. The possibility of an enhanced carbon and/or nitrogen abundance was considered unlikely. Burstein (1987) later showed that $\mathrm{K}$ giants contributing to the integrated light of M 31 clusters must have at least as strong $\mathrm{CN}$ absorption bands as the most extreme Galactic giant stars. In a subsequent analysis of the CaII $\mathrm{H}+\mathrm{K}$ absorption feature of nine metal-rich M 31 globular clusters, Tripicco (1989) rejected the hypothesis that hot horizontal-branch stars and/or blue stragglers boost the $\mathrm{H} \beta$ index of the integrated light. Modeling the spectra of M 31 clusters he also found that the strong $\mathrm{CN}$ index is consistent with roughly a factor of 10 in $\mathrm{CN}$ excess in both dwarf and giant stars. Davidge (1990a) obtained near-infrared spectra of four luminous M 31 globular clusters and compared them with Galactic counterparts. He found a clear enhancement in the $2 \mu \mathrm{m} \mathrm{CN}$ band and concluded that the increased CN abundance in M 31 globular clusters is likely to be of primordial origin. From a comparison of $\mathrm{CO}$ absorption bands in M 31 clusters and the integrated light of M 32, which is known to host an intermediate-age population (e.g. Davidge 1990b), he reasoned that, if both stellar populations have similar metallicities, luminous M 31 globular clusters may contain an intermediate-age component.

In a comprehensive spectroscopic investigation of 149 globular clusters in M 31, using specifically defined line indices, Brodie \& Huchra (1990) confirmed a CN offset relative to Milky Way globular clusters, but failed to identify a stronger mean $\mathrm{H} \beta$ index, although good agreement was found between the measurements of matched objects. This fact suggests that sample selection in the M 31 cluster system might be playing an important role and implies the presence of multiple globular cluster sub-populations with different mean $\mathrm{H} \beta$ indices and potentially different chemical compositions. Brodie \& Huchra reported also an enhancement in their CaII $\mathrm{H}+\mathrm{K}$ index for $\mathrm{M} 31$ globular clusters, at a given metallicity. Assuming that the $\mathrm{CN}$ enhancement is due to an increased nitrogen abundance they identify aluminum satellite lines as responsible for an increased $\mathrm{H}+\mathrm{K}$ index, because of the correlation of nitrogen with aluminum abundance, at least in metal-rich stars (Norris \& Pilachowski 1985).

An alternative explanation for the observed $\mathrm{CN}$ offset is lower mean surface gravities, as these elevate both $\mathrm{CN}$ and CaII H+K (O'Connell 1973). The surface gravity can be lowered by decreasing the age; however, other indices such as $\mathrm{Mg}_{2}$ (a measure of the Mgb triplet and the magnesium hydride band, $\mathrm{MgH}$ ) increase significantly as age is decreased, and such an increase is not observed. However, the $\mathrm{Mg}_{2}$ index can be kept constant if the intrinsic magnesium abundance and age are both decreased appropriately. The former could be a result of a low $[\alpha / \mathrm{Fe}]$ ratio at constant total metallicity. This is expected for stellar populations which formed late from material enriched by type II and Ia supernovae (e.g. Matteucci 1994; Matteucci \& Recchi 2001). Later work using near-UV spectra analyzed the strength of the NH band at $3360 \AA$ (closely linked to the nitrogen abundance) for several M 31 globular clusters (Ponder et al. 1998; Li \& Burstein 2003; Burstein et al. 2004). These studies confirmed that M 31 globular clusters are indeed enhanced in nitrogen.

Furthermore, previous studies (Barmby et al. 2000; Beasley et al. 2004; Burstein et al. 2004) showed the presence of young M 31 globular clusters. These young objects have spectra which resemble those of young globular clusters in the Large Magellanic Cloud with ages around 1 Gyr. Some of these M 31 clusters appear to belong to a distinct sub-population of thin disk globular clusters discovered by Morrison et al. (2004).

It is obvious that a study of ages and chemical compositions for a large sample of M 31 globular clusters is urgently needed to resolve some of the above issues. Previous studies have shown indications of young ages for some M 31 globular clusters and clear signatures of chemical differences between the globular cluster system in M 31 and other galaxies.

In this paper, we focus on the analysis of spectroscopic features of M 31 globular clusters using Lick line indices from which we derive ages, metallicities, and abundance ratios. Newly available simple stellar population (SSP) models for well-defined chemical compositions (Thomas et al. 2003, 2004) make these parameters accessible with unprecedented systematic accuracy. We use high-quality Lick index 
measurements and a new iterative prescription to derive these parameters that maximally reduce systematic uncertainties in the internal calibration of Lick indices.

The paper is organized as follows: Sect. 2 describes the dataset and its calibration to the Lick system. In the following Sect. 3 we describe our method and perform a consistency check for ages and metallicities derived from different diagnostic grids. Results on M 31 globular cluster ages, metallicities, and abundance ratios are presented in Sect. 4, along with a comparison with globular cluster systems in other galaxies. Our findings are discussed in Sect. 5 and summarized in Sect. 6.

\section{Data}

\section{1. globular cluster spectra}

We use spectroscopic observations obtained with the Wide Field Fibre Optic Spectrograph (WYFFOS, Bridges 1998) at the $4 \mathrm{~m}$ William Herschel Telescope on La Palma, Canary Islands. The dataset is described in detail by Perrett et al. (2002) and we refer the reader to this paper for a discussion of technical details. Figure 1 shows the field of view with all fiber positions. Open symbols indicate globular clusters; crosses mark the position of background fibers. Sky corrections for globular cluster spectra were performed by subtracting a mean sky spectrum that was constructed from several nearby fibers placed in close vicinity to each individual globular cluster. Throughout the following analysis we use these sky-subtracted spectra.

\subsection{Spectra of the diffuse light}

In addition to our globular cluster measurements (see open symbols in Fig. 1), we also obtained 207 integrated spectra of the diffuse stellar population at different galactocentric radii. Each individual spectrum has too low a signal-to-noise $(S / N)$ to allow reliable Lick index measurements. In order to maximize the $S / N$ ratio, we select spectra from the inner Bulge field (within $5 \mathrm{kpc}$ projected galactocentric distance) and subdivide this background sub-sample into an inner and outer sector, $z 0$ and $z 1$, respectively. The radial sampling of the combined two sectors is described in Table 1. Individual spectra in each sector are then co-added. Before summing the spectra, a careful smoothing to the Lick resolution of each single spectrum was performed, accounting for the varying velocity dispersion at each individual fiber position and the changing spectral resolution as a function of chip position. Velocity dispersions as a function of galactocentric distance were calculated with the disk-bulge-halo models of Widrow et al. (2003). The average $S / N$ ratio of both combined background spectra exceeds $\sim 25$ per $\AA$.

\subsection{Calibration of lick indices}

Prior to the measurement of Lick line indices, our spectra were broadened to the characteristic Lick resolution. The steps undertaken during this procedure were adopted from Worthey \& Ottaviani (1997); Kuntschner (1998, 2000); Vazdekis \& Arimoto (1999); Puzia et al. (2002b, 2004a), and
Table 1. Radial coverage of background sectors. $r_{\min }, r_{\max }$, and $r_{\text {median }}$ are the radii of the fiber pointings with the minimal and maximal galactocentric distance, and the median distance of the entire sub-sample.

\begin{tabular}{lccc}
\hline \hline Sector & $r_{\min }[\mathrm{kpc}]$ & $r_{\max }[\mathrm{kpc}]$ & $r_{\text {median }}[\mathrm{kpc}]$ \\
\hline$z 0$ & 0.55 & 0.85 & 0.60 \\
$z 1$ & 1.56 & 4.96 & 2.48 \\
\hline
\end{tabular}

Table 2. Correction terms of the transformation to match the Lick/IDS instrumental resolution. The corrections were used to transform our index measurements to the Lick/IDS system Worthey et al. (1994), in the sense $I_{\text {Lick }}=I_{\text {measured }}+c$.

\begin{tabular}{lrc}
\hline \hline Index & $c$ & rms \\
\hline $\mathrm{H} \delta_{A}$ & -0.127 & 2.041 \\
$\mathrm{H} \delta_{F}$ & -0.437 & 0.697 \\
$\mathrm{CN}_{1}$ & 0.001 & 0.096 \\
$\mathrm{CN}_{2}$ & 0.051 & 0.084 \\
$\mathrm{Ca} 4227$ & -0.407 & 0.956 \\
$\mathrm{G} 4300$ & -0.315 & 3.059 \\
$\mathrm{H} \gamma_{A}$ & -0.488 & 0.514 \\
$\mathrm{H} \gamma_{F}$ & -0.094 & 1.106 \\
$\mathrm{Fe} 4383$ & 0.764 & 2.480 \\
$\mathrm{Ca} 4455$ & 0.541 & 0.796 \\
$\mathrm{Fe} 4531$ & 1.086 & 1.445 \\
$\mathrm{Fe} 4668$ & 0.007 & 2.193 \\
$\mathrm{H} \beta$ & 0.148 & 0.175 \\
$\mathrm{Fe} 5015$ & -0.300 & 1.547 \\
$\mathrm{Mg}_{1}$ & 0.004 & 0.025 \\
$\mathrm{Mg}_{2}$ & 0.025 & 0.041 \\
$\mathrm{Mgb}$ & -0.123 & 0.695 \\
$\mathrm{Fe} 5270$ & 0.730 & 0.589 \\
$\mathrm{Fe} 5335$ & 0.243 & 0.576 \\
$\mathrm{Fe} 5406$ & -0.064 & 0.549 \\
$\mathrm{Fe} 5709$ & 0.355 & 0.489 \\
\hline
\end{tabular}

Puzia (2003b). A particular characteristic of the WYFFOS instrument is its resolution as a function of chip position. To account for this, we computed 2-dim. resolution maps using arc exposures that were taken close in time with the science frames. The resolution maps were then used to smooth our spectra to the differential Lick resolution of $\gtrsim 8 \AA$ (see Worthey $\&$ Ottaviani 1997, for details). We tested this transformation by matching our globular cluster list with the M 31 globular cluster sample of Worthey et al. (1994) and Kuntschner et al. (2002). The comparison gives six matches (B012-G64, B015, B193-G244, B218-G272, B225-G280, B311-G33) that cover a range in $[\mathrm{Fe} / \mathrm{H}]$ from -2.0 to -0.3 dex. Small linear correction terms were found for all indices, that in all cases had a significance of $\lesssim 1 \sigma$ (see Table 2). These systematic offsets were applied to all subsequent Lick index measurements ${ }^{1}$.

1 Note that bad pixel masking leaves two out of the six spectra useful for calibration of the $\mathrm{H} \gamma_{A}$ index. The two remaining good-quality spectra are in very good agreement with the Lick standard measurements. However, we warn that the rms of this calibration cannot be trusted given the small sample. 


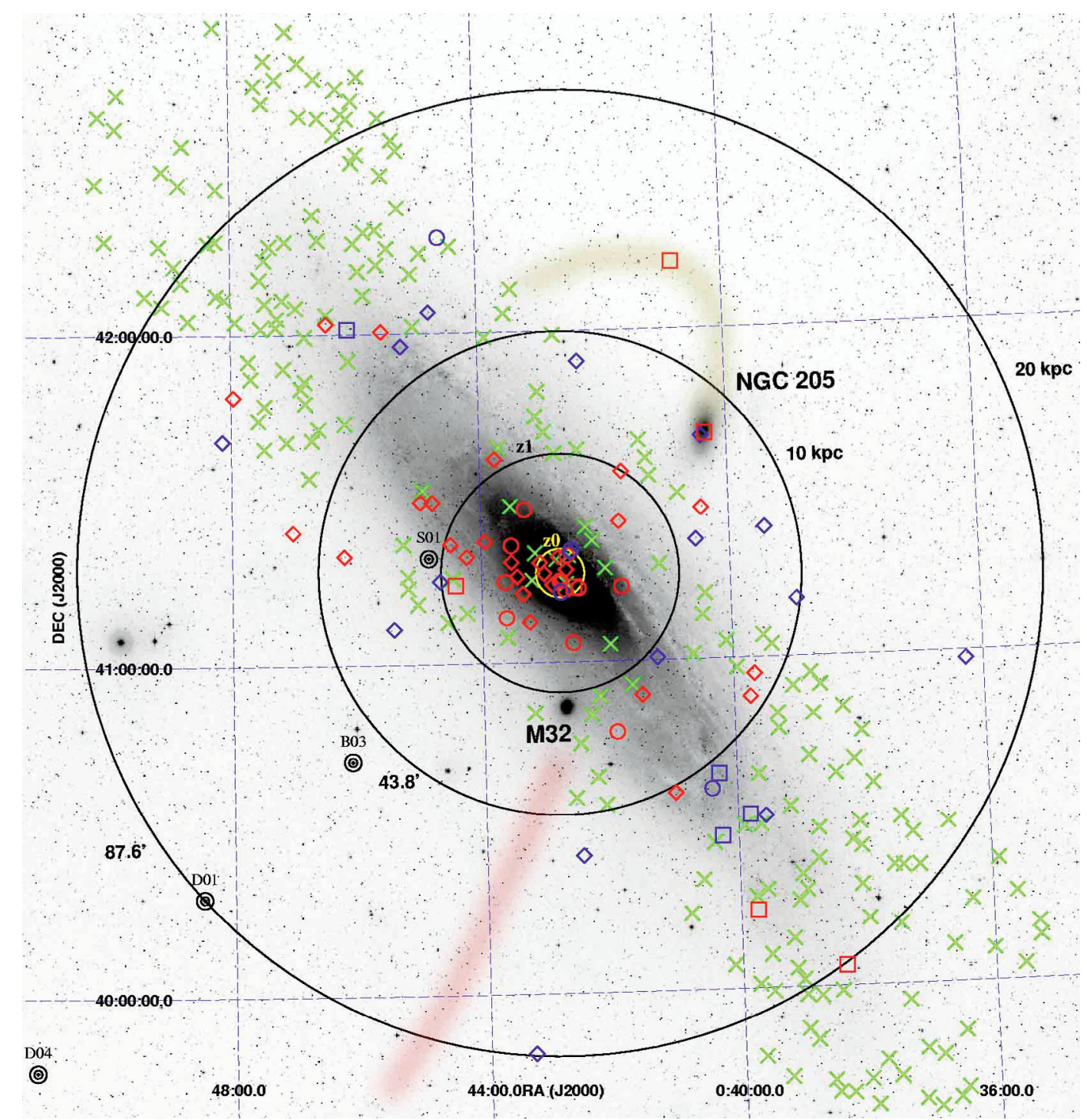

Fig. 1. Positions on the sky of studied globular clusters (open symbols) and background fields (crosses). Globular clusters are parameterized by their age and metallicity. Squares indicate globular clusters with ages below $5 \mathrm{Gyr}$, circles show intermediate-age globular clusters with ages between 5 and $8 \mathrm{Gyr}$, while diamonds mark the position of old globular clusters $(t>8 \mathrm{Gyr})$. Old objects populate the entire studied field. Intermediate-age globular clusters seem to prefer the region within the central $\sim 5 \mathrm{kpc}$, while young clusters are preferentially located in the outer disk. The metallicity is indicated by color-coded symbols. Red symbols show metal-rich globular clusters with $[\mathrm{Z} / \mathrm{H}]>-0.8$, while blue symbols show their metal-poor counterparts. Note the clear concentration of metal-rich objects around the nucleus of M 31. Background spectra were grouped in sectors $z 0$ and $z 1$ according to their galactocentric distance indicated by the two inner circles. A few globular clusters (e.g. MII = G1) which are located at large galactocentric distances fall off the image, similar to some background pointings. The underlying image was taken from DSS2/red. Shaded trails indicate stellar streams discovered by the INT/CFHT collaboration (see Ibata et al. 2001; Ferguson et al. 2002; McConnachie et al. 2004). While the southern stream is the most massive $\left(\sim 10^{8}-10^{9} M_{\odot}\right)$, the northern spur carries about an order of magnitude less mass and appears to be kinematically associated with NGC 205 (McConnachie et al. 2004). Reticles mark pointings for which deep photometry is available. Going from small to larger galactocentric distances the fields are labeled as S01 (Sarajedini \& Van Duyne 2001), B03 (Brown et al. 2003), D01 (Durrell et al. 2001), and D04 (Durrell et al. 2004).

Blue Lick indices (from $\mathrm{CN}_{1}$ to Fe4383) were measured on spectra taken with the blue arm of WYFFOS, which covers the spectral range $\sim 3700-4500 \AA$. The remaining Lick indices (Ca4455 to Fe5709) were measured on spectra taken with the red-arm with a spectral coverage from $\sim 4400$ to $5800 \AA$. Spectra with $S / N$ of $\sim 25$ per $\AA$ or less were rejected from the subsequent analysis. As we propagate the full error budget of the spectra into the line index measurement, some indices show unusually large error bars due to masked bad pixels and/or cosmics. These contaminated index measurements were also rejected from the following analysis steps. With these data quality requirements 29 globular clusters remain in our sample.

\subsection{Data from other sources}

To maximize the statistical significance of the globular cluster sample, we include five M 31 globular clusters from the Lick group (Worthey et al. 1994; Worthey \& Ottaviani 1997) and 


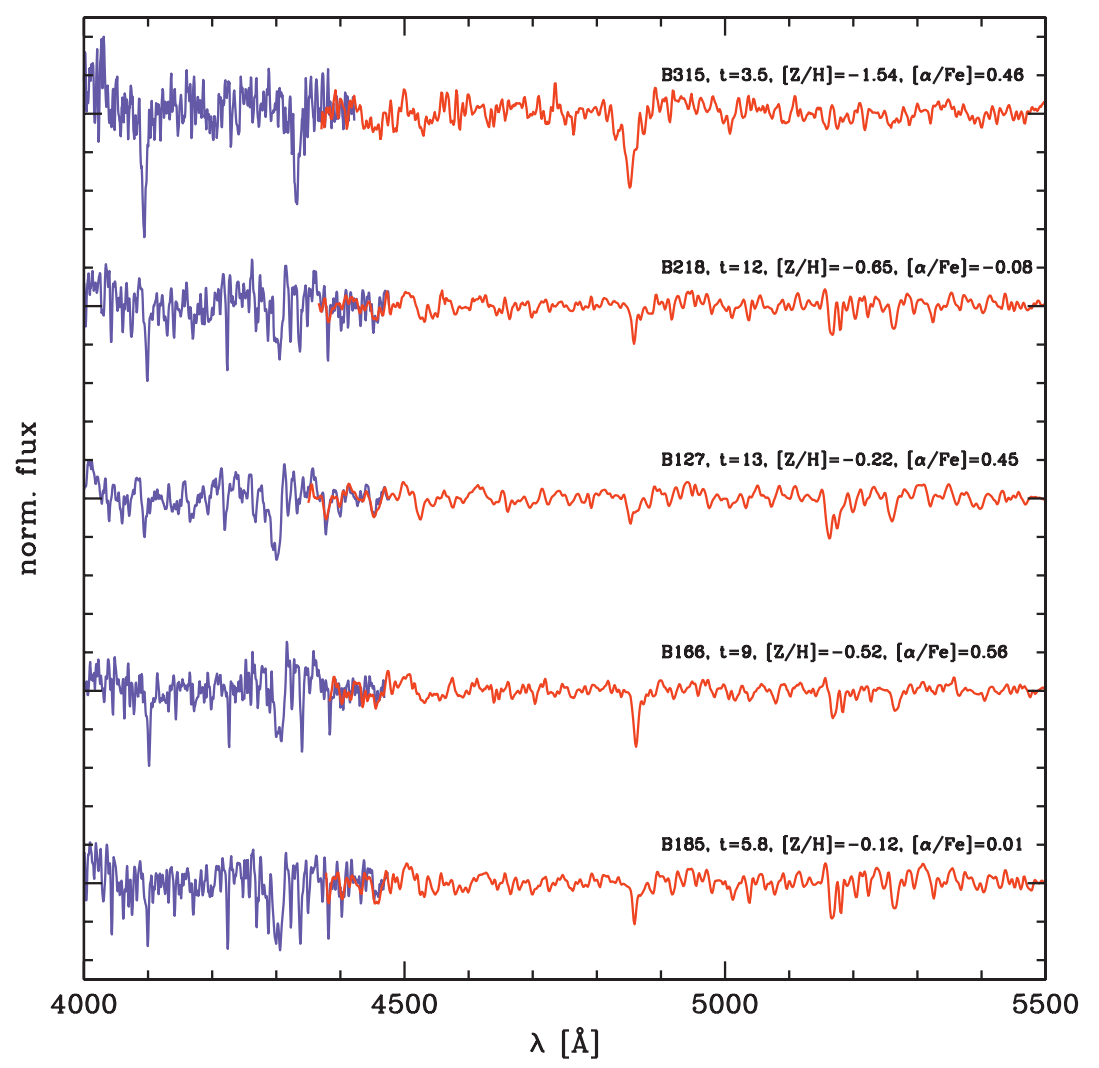

Fig. 2. Representative spectra from our M 31 globular cluster sample for selected targets, sorted by metallicity (increasing from top to bottom). Blue and red spectra are plotted separately. Derived ages (in Gyr), metallicities, and $[\alpha / \mathrm{Fe}]$ ratios from the subsequent analysis steps are indicated.

36 globular clusters from the Beasley et al. (2004) sample. We note that all index measurements are performed in the same passband system as the SSP models, in particular the fitting functions that are used to compute theoretical predictions.

We find two matches between our globular cluster data and the Beasley et al. dataset. The Lick index measurements for the two clusters, i.e. B225 and B243, are in very good agreement (within 1- $\sigma$ ), especially for the widely-defined molecular-band indices (e.g. $\mathrm{CN}_{2}$ and $\mathrm{Mg}_{2}$, but not for $\mathrm{G} 4300^{2}$ ). Very good agreement is also found for indices crucial to further analysis steps, such as Balmer, iron, and magnesium indices: $\mathrm{H} \delta_{A}, \mathrm{H} \gamma_{A}$, $\mathrm{H} \beta, \mathrm{Mg}_{2}, \mathrm{Mgb}, \mathrm{Fe} 5270, \mathrm{Fe} 5335$, etc. Because of the smaller statistical errors of the Beasley et al. data we adopt the latter for the following analysis. Hence our final dataset contains highquality spectra $\left(S / N \gtrsim 25 \AA^{-1}\right)$ for 70 globular clusters in M 31 . The full set of index measurements is presented in Tables A.1 and A. 2 in the Appendix. Note that object B166 is suspected to be a foreground star (Galleti et al. 2004). However, since this is not certain, and because we do not find any features in our spectrum that would be characteristic of a foreground star, we mark this object as potentially misclassified but keep it in our globular cluster sample. Note that based on their colors and radial velocities, all other objects were classified as genuine globular

${ }^{2}$ We find a $\sim 1.5 \AA$ systematic offset towards higher values in the Beasley et al. sample, although one of our measurements has a very large error of $1.2 \AA$. clusters by several independent studies (see Galleti et al. 2004). Figure 2 shows representative spectra from our sample.

We do not include indices from Burstein et al. (1984) since they were measured in the old Lick passband system, for which no model predictions with variable abundance ratios are available. However, we find eight matched M 31 clusters in our and the Trager et al. (1998) data, who re-measured Lick indices for M 31 and Milky Way globular clusters from the Burstein et al. sample with slightly modified passband definitions compared to the ones used in this work ${ }^{3}$. We therefore briefly investigate the previously detected $\mathrm{H} \beta$ offset between Galactic and M 31 globular clusters in the Burstein et al. (1984) study. In Fig. 3 we reproduce the $\mathrm{H} \beta$ and $\mathrm{CN}_{2}$ vs. $\mathrm{Mg}_{2}$ plots from Burstein et al. (1984). We include only the eight M 31 globular clusters that overlap with the Trager et al. data and were re-observed in this study (solid circles) and augment the sample with clusters from the Trager et al. (1998) study that meet our quality selection criteria (five open circles). Also included are 12 Galactic globular clusters from Puzia et al. (2002b) (solid squares), and 13 clusters from Trager et al. (1998) (open squares).

\footnotetext{
3 The difference in passband definitions between the ones adopted in this work (Worthey et al. 1994; Worthey \& Ottaviani 1997) and the ones of (Trager et al. 1998) is significant for some indices. Linear transformations between the two systems were computed by Puzia et al. (2004a). $\mathrm{H} \beta$ has identical definitions in both systems. For the $\mathrm{CN}_{2}$ the offset is $\Delta \mathrm{CN}_{2}(\operatorname{Tr} 98-$ Wo94)0.014 \pm 0.020 mag. Where applicable, these linear corrections are applied to the Trager et al. data.
} 

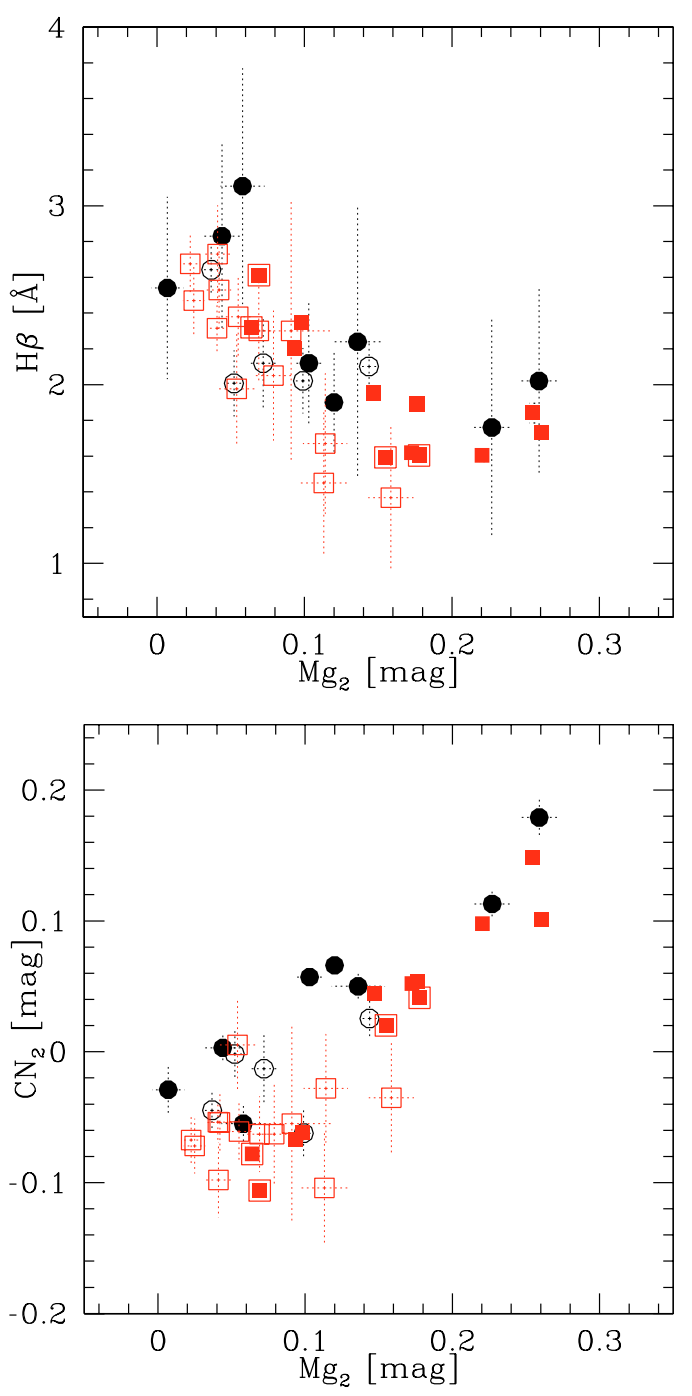

Fig. 3. $\mathrm{H} \beta$ and $\mathrm{CN}_{2}$ vs. $\mathrm{Mg}_{2}$ plots for $\mathrm{M} 31$ and Galactic globular clusters. Milky Way globular clusters are indicated as squares, while M 31 globular clusters are marked by circles. Solid squares show data taken from Puzia et al. (2002b). Open squares are Galactic globular clusters from Trager et al. (1998). Large open squares illustrate matches between the Puzia et al. and Trager et al. dataset. Solid circles are M 31 globular clusters included in the Burstein et al. dataset that were remeasured in this study. Open circles add M 31 data from Trager et al. (1998). See text for details.

We find four matches between the Galactic cluster samples of Puzia et al. and Trager et al., and plot for matched objects the indices measured by Puzia et al., because of their smaller errors. The systematic offsets for the four matches in the sense Puzia et al. - Trager et al. are $\Delta \mathrm{H} \beta=0.31 \pm 0.28 \AA$ and $\Delta \mathrm{CN}_{2}=-0.02 \pm 0.04 \mathrm{mag}$. The matched objects are marked with large open squares. From the eight matched M 31 globular clusters between our sample and that of Trager et al. we find in general very good agreement between the two index measurements for all relevant indices, such as Balmer, $\mathrm{Mg}$, and $\mathrm{Fe}$ indices. In particular, the offsets, in the same sense as above, are $\Delta \mathrm{H} \beta=0.06 \pm 0.13 \AA$ and $\Delta \mathrm{CN}_{2}=0.026 \pm 0.023 \mathrm{mag}$. The systematic offset in $\mathrm{H} \beta$ is clearly less significant than the one found between the Galactic cluster samples.
Although the error bars of the M 31 sample are relatively large, there is no significant systematic offset between M 31 and Milky Way globular clusters in the newly acquired data (solid symbols). Fitting straight lines to the new data in the $\mathrm{H} \beta$ vs. $\mathrm{Mg}_{2}$ plot yields the following linear relations:

M $31: \mathrm{H} \beta=(-4.05 \pm 1.03) \cdot \mathrm{Mg}_{2}+(2.58 \pm 0.18)$

$\mathrm{MW}: \mathrm{H} \beta=(-3.95 \pm 1.50) \cdot \mathrm{Mg}_{2}+(2.78 \pm 0.22)$.

The rms for both relations are 0.30 and $0.21 \AA$ for M 31 and Galactic globular clusters. Since the gradients are virtually identical, the zero-point offset is a measure of the systematic offset. It is smaller than the mean rms of both relations. If we split the datasets at $\mathrm{Mg}_{2}=0.2 \AA$, the systematic offsets for both high and low- $\mathrm{Mg}_{2}$ subsets disappear, i.e. they are less significant than $\sim 0.5 \sigma$. However, if the Galactic globular clusters from Trager et al. (small and large open squares) are considered, we confirm a systematic offset $\Delta \mathrm{H} \beta \approx 0.5 \AA$ $(\gtrsim 3 \sigma)$ at $\mathrm{Mg}_{2} \gtrsim 0.1 \mathrm{mag}$ between M 31 and Milky Way (see also Fig. 5k in Burstein et al. 1984). In summary, a combination of sample selection, small number statistics, and a potential calibration inconsistency might have introduced spurious $\mathrm{H} \beta$ offsets between Galactic and M 31 globular clusters in the Burstein et al. work.

The new data do not appear to alter the conclusion of Burstein et al. on the $\mathrm{CN}$ offset. However, for metal-rich globular clusters the offset in $\mathrm{CN}$ seems smaller than for metalpoor globular clusters. In general, this exercise demonstrates that for drawing reliable conclusions from a direct comparison of datasets, a homogeneous and consistent sample selection of these datasets is crucial.

Later in this work we show that the speculation of Burstein et al. that the $\mathrm{H} \beta$ offset was produced by a systematically younger mean age of their observed M 31 globular cluster sample is likely to be the result of Milky Way globular cluster sample selection. From their sample of 19 M 31 globular clusters, 16 had their indices on high-quality spectra re-measured in subsequent studies. In fact, in this study we find that the mean age of this M 31 sub-sample is $\sim 10 \mathrm{Gyr}$ with only four globular clusters between 6 and 9 Gyr. However, their intuition that M 31 might host young globular clusters turns out to be correct, if a larger more representative sample of M 31 globular clusters is considered.

\section{Analysis}

\subsection{Comparison with SSP Models}

In the following section we compare line index measurements for M 31 globular clusters and background fields with predictions of SSP models, which were calculated for stellar populations with well-defined $[\alpha / \mathrm{Fe}]$ abundance ratios (Thomas et al. 2003, 2004). These models are based on Worthey et al. (1994) and Worthey \& Ottaviani (1997) fitting functions, response functions from Tripicco \& Bell (1995) and Korn et al. (2004, in preparation), solar-scaled stellar evolutionary tracks, and employ the principles of the fuel consumption theorem (Renzini \& Buzzoni 1986). 
Spectroscopic ages and metallicities of unresolved globular clusters are traditionally derived from Balmer line indices and a combination of metal indices. According to Puzia (2003a) and Puzia et al. (2004b) the best combination of Lick indices to construct an age-metallicity diagnostic diagram depends on internal uncertainties of the Lick system and the data quality. In our case the most reliable combination is $\mathrm{H} \gamma_{A}$ and $[\mathrm{MgFe}]^{\prime 4}$. The combination of magnesium and iron-sensitive indices, in the form of the composite index [MgFe]', is an excellent metallicity tracer (Puzia et al. 2002b) and shows the weakest sensitivity to $[\alpha / \mathrm{Fe}]$ abundance ratio variations (Thomas et al. 2003). Among all Balmer Lick indices, $\mathrm{H} \gamma_{A}$, followed by $\mathrm{H} \beta$ and $\mathrm{H} \delta_{A}$, are least affected by internal systematics, such as systematic uncertainties in the fitting functions. However, we note that all Balmer indices are influenced by abundance ratio variations (e.g. $\alpha / \mathrm{Fe}$ ), in particular the higher-order Balmer indices. Although response functions exist only for a selected set of elements (Tripicco \& Bell 1995), with the new SSP model predictions these variations are under control to first order.

Considering the signal-to-noise of a dataset, the accuracy of the calibration to the Lick system, and internal systematic uncertainties of Balmer Lick indices, Puzia et al. (2004a) computes a reliability parameter $\mathcal{R}$, that indicates the quality of a Balmer Lick index as an age indicator. Based on this classification we have decided to derive ages and metallicities from diagnostic diagrams constructed from the Balmer indices $\mathrm{H} \gamma_{A}$, $\mathrm{H} \beta$, and $\mathrm{H} \delta_{A}$, only. The narrowly-defined Balmer indices, $\mathrm{H} \gamma_{F}$ and $\mathrm{H} \delta_{F}$, have low signal-to-noise and measure the same absorption features as $\mathrm{H} \gamma_{A}$ and $\mathrm{H} \delta_{A}$ (hence, do not provide additional information). They have the lowest reliability parameter $\mathcal{R}$ among all Balmer indices (see Puzia et al. 2004a), so that we refrain from including them in subsequent analyses.

\subsection{Fitting routine}

To derive reliable ages and metallicities, we need to utilize a diagnostic grid from which the $[\alpha / \mathrm{Fe}]$ ratio of the observed stellar population can be derived. We tested several combinations of Lick indices and, based on the internal systematics, the relative separation of iso- $[\alpha / \mathrm{Fe}]$ tracks, and the mean measurement error, decided on an $[\alpha / \mathrm{Fe}]$ diagnostic grid that is constructed from the indices $\mathrm{Mg}_{2}$ and $\langle\mathrm{Fe}\rangle$. The $\mathrm{Mg}_{2}$ index measures the strength of the $\mathrm{Mgb}$ and $\mathrm{MgH}$ features at $\sim 5100 \AA$. The mean iron index $\langle\mathrm{Fe}\rangle$ is a composite index built from the indices $\mathrm{Fe} 5270$ and $\mathrm{Fe} 5335$, i.e. $\langle\mathrm{Fe}\rangle=\sqrt{\mathrm{Fe} 5270+\mathrm{Fe} 5335}$, which trace two strong iron lines at similar wavelengths $(\lambda 5270,5335 \AA)$ as $\mathrm{Mg}_{2}$. However, since the $[\alpha / \mathrm{Fe}]$ diagnostic grid itself is degenerate in age and metallicity, the best possible determination of ages, metallicities, and $[\alpha / \mathrm{Fe}]$ ratios requires an iterative fitting of the data, feeding information acquired from the $\alpha / \mathrm{Fe}$-grid into the age/metallicity-grid.

Extensive tests have shown that the values derived from both diagnostic diagrams converge after a few iterations. Our routine starts with the determination of $[\alpha / \mathrm{Fe}]$ ratios from the $\mathrm{Mg}_{2}$ vs. $\langle\mathrm{Fe}\rangle$ diagram, assuming a $13 \mathrm{Gyr}$ old population, and is based on an $\chi^{2}$ minimization technique. The age and

\footnotetext{
${ }^{4}[\mathrm{MgFe}]^{\prime}=\sqrt{\mathrm{Mgb}(0.72 \cdot \mathrm{Fe} 5270+0.28 \cdot \mathrm{Fe} 5335)}$.
}

metallicity of each globular cluster is then determined with the same $\chi^{2}$ minimization approach on an individually interpolated ${ }^{5} \mathrm{H} \gamma_{A}, \mathrm{H} \beta$, and $\mathrm{H} \delta_{A}$ vs. [MgFe]' grid for a given $[\alpha / \mathrm{Fe}]$. For globular clusters with index measurements that do not fall on the diagnostic grid, we assign the most likely value, extrapolated along the most likely error vector which is constructed from the square sum of individual index uncertainties for each globular cluster in a given diagnostic grid. The age and metallicity values are then fed back into the $[\alpha / \mathrm{Fe}]$ diagram and the process is iterated. In most cases this process requires only a few cycles for the values to converge. This method is superior to any age-metallicity determination based on the agemetallicity grid only. As shown in Fig. 4, the isochrones of the age-metallicity grid can change significantly up to a few typical 1- $\sigma$ measurement errors as a function of $[\alpha / \mathrm{Fe}]$ (see also Thomas et al. 2003, 2004). To control these effects and obtain meaningful age/metallicity results it is imperative to include abundance ratio variations in each age-metallicity determination based on Lick index measurements.

Ages, metallicities, and $[\alpha / \mathrm{Fe}]$ ratios for all M 31 sample globular clusters are summarized in Table A.3 in the Appendix.

\subsection{Consistency check}

In Fig. 5 we compare age and metallicity determinations from the three diagnostic grids using different Balmer-line indices, $\mathrm{H} \gamma_{A}, \mathrm{H} \beta$, and $\mathrm{H} \delta_{A}$, as age indicators (see Fig. 6). The rms scatter in the comparison of ages derived from two different grids varies between 3.6 and 4.7 Gyr. Note that the age scatter increases from young to old ages, which is an effect of an increasing age resolution with decreasing absolute age. Given the quality of our dataset, the rule of thumb for the average age uncertainty is $\Delta t / t \approx 1 / 3$. The consistency check shows that the rms-scatter in all upper panels of Fig. 5 is in most cases comparable or larger than the mean measurement error. This is an indication for the presence of systematic offsets in the absolute age scale between two diagnostic grids. The rms-scatter in all metallicity consistency plots agrees very well with the mean measurement error, and shows the good absolute metallicity calibration of individual diagnostic grids. It is not too surprising that the metallicity values derived from different grids agree very well for individual globular clusters, since all plots use $[\mathrm{MgFe}]^{\prime}$ as metallicity proxy. A good estimate for the mean metallicity uncertainty is 0.2 dex.

The consistency shown between the model predictions should be taken as a demonstration of the quality of age/metallicity predictions that are feasible with today's highquality datasets and SSP models. Rather than being an endorsement of the particular model used in this study, we point out that SSP model predictions for stellar populations with welldefined abundance ratios are essential to reach the same quality of age/metallicity determinations as presented here. Other SSP models which do not take the systematics of abundanceratio variations into account may lead to similarly good fits for a given diagnostic grid, but the results will suffer from

\footnotetext{
5 We linearly interpolate the grid in age and $Z$-space (not metallicity $\log$-space).
} 

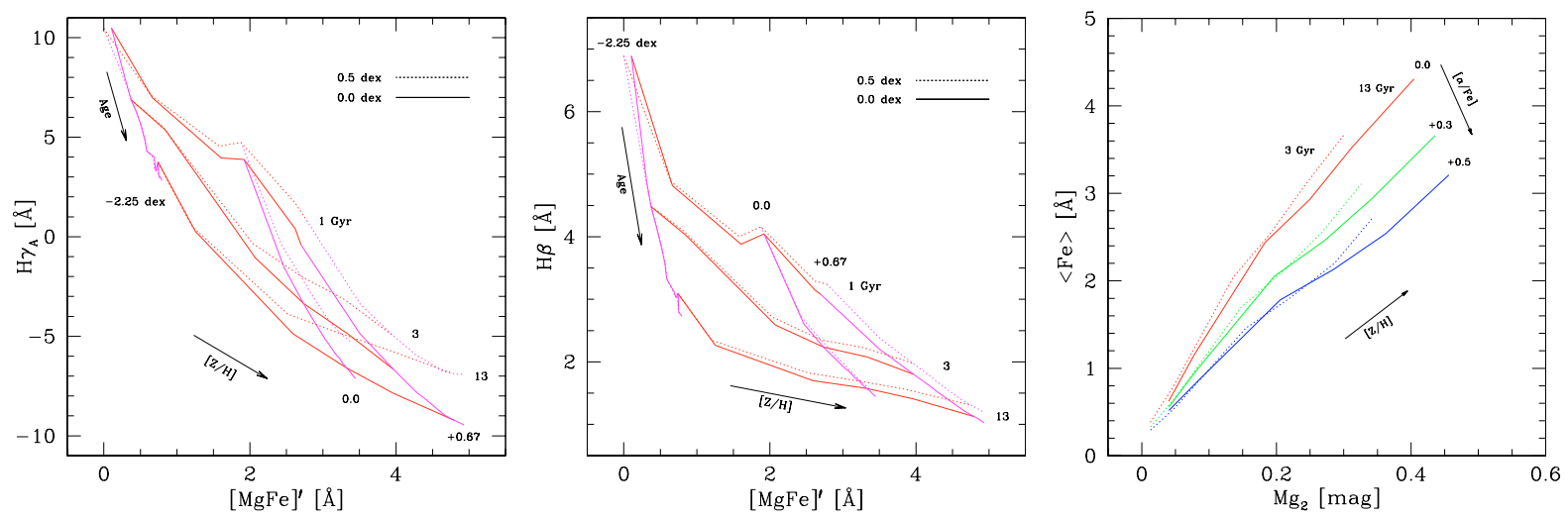

Fig. 4. Comparison of diagnostic grids for Lick index measurements, parameterized for different $[\alpha / \mathrm{Fe}]$ abundance ratios and ages. Model predictions were taken from Thomas et al. (2003, 2004). The left and middle panel show two grids constructed from [MgFe]' as a metallicity indicator and two different Balmer line indices as age indicators, i.e. $\mathrm{H} \gamma_{A}$ and $\mathrm{H} \beta$, respectively. The plots show models grids for two different values of $[\alpha / \mathrm{Fe}]=0.0$ (solid lines) and $0.5 \mathrm{dex}$ (dashed lines). Note the increasing differential offset between the grids towards higher metallicities. The right panel shows the $[\alpha / \mathrm{Fe}]$ diagnostic diagram, which features the $\mathrm{Mg}_{2}$ index as a representative $\alpha$-element indicator and $\langle\mathrm{Fe}\rangle$ as a iron-abundance proxy. The diagram shows the dependence of iso- $[\alpha / \mathrm{Fe}]$ as a function of age. Dotted lines show the model predictions for 3 Gyr old populations and solid lines for a 13 Gyr counterpart.
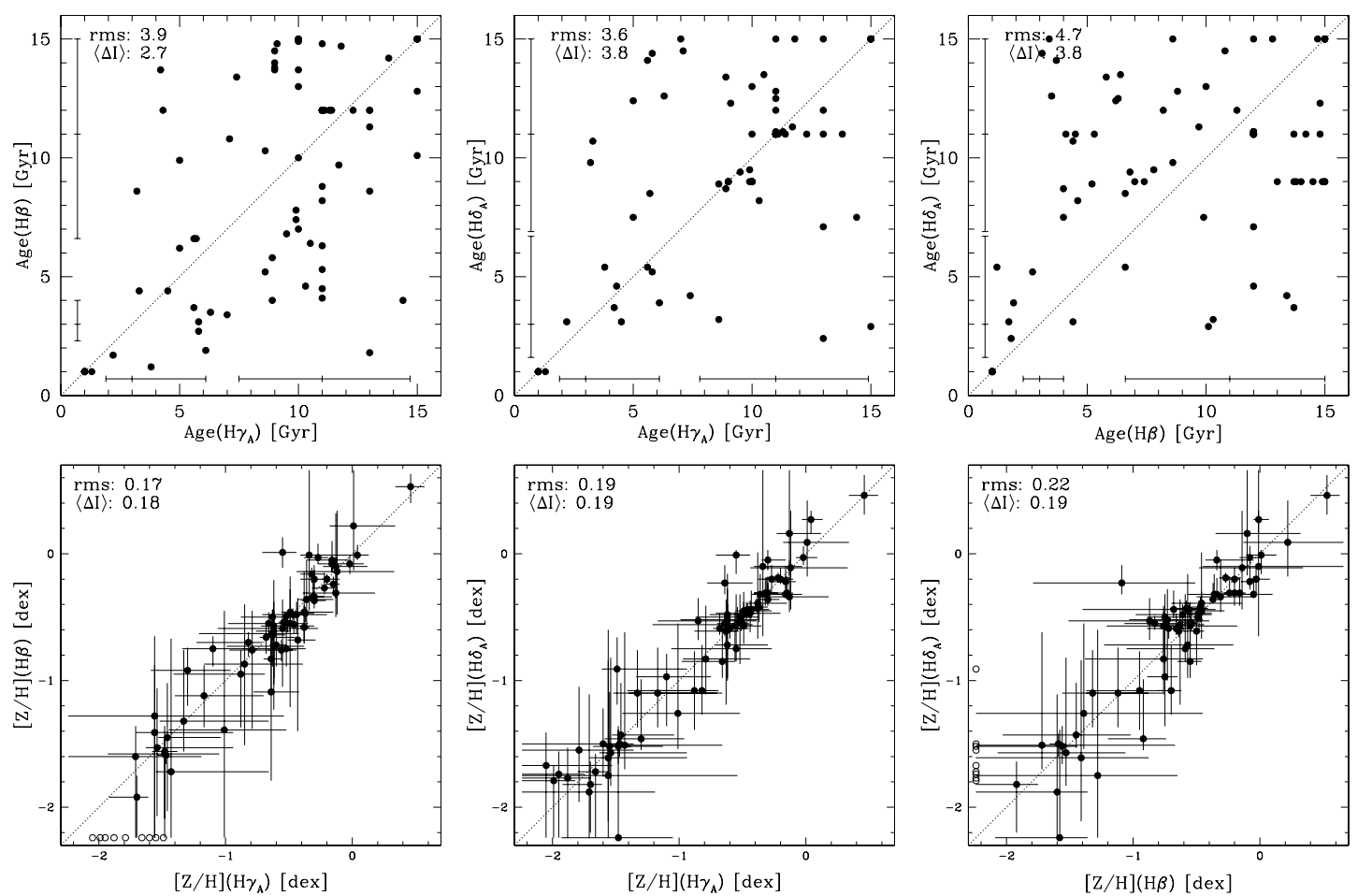

Fig. 5. Comparison of age and metallicity predictions from the three different age-metallicity diagnostic grids shown in Fig. 6. Upper panels show the comparison of ages; lower panels illustrate the comparison of metallicity values. The rms scatter is indicated in the upper left corner of each plot and is the scatter around the one-to-one relation. $\langle\Delta I\rangle$ indicates the median error for this panel. In the upper panels median error bars are indicated at the abscissa and ordinate, calculated as the sample median of the averaged globular cluster asymmetric error. Open circles mark outliers which were not included in the rms determination.

systematic uncertainties described earlier. However, even for state-of-the-art SSP models the litany of systematic uncertainties is long 6 .

${ }^{6}$ Systematic uncertainties are still hard-wired into all current SSP Lick-index predictions and might, if accounted for, significantly improve future age, metallicity, and abundance ratio estimates. To name a few "to-dos": control of systematic uncertainties introduced by variations in horizontal branch morphology, self-consistent use of enhanced
In general, we find good congruence between age and metallicity predictions between diagnostic plots using the three indices $\mathrm{H} \delta_{A}, \mathrm{H} \gamma_{A}$, and $\mathrm{H} \beta$. The relative age accuracy ( $\left.\sim 3-5 \mathrm{Gyr}\right)$ allows us to disentangle old from intermediate-age and young

stellar evolutionary tracks/isochrones in SSP models, extension of response functions to more elements, self-consistent calculation of internal energy production for various chemical compositions, etc. 

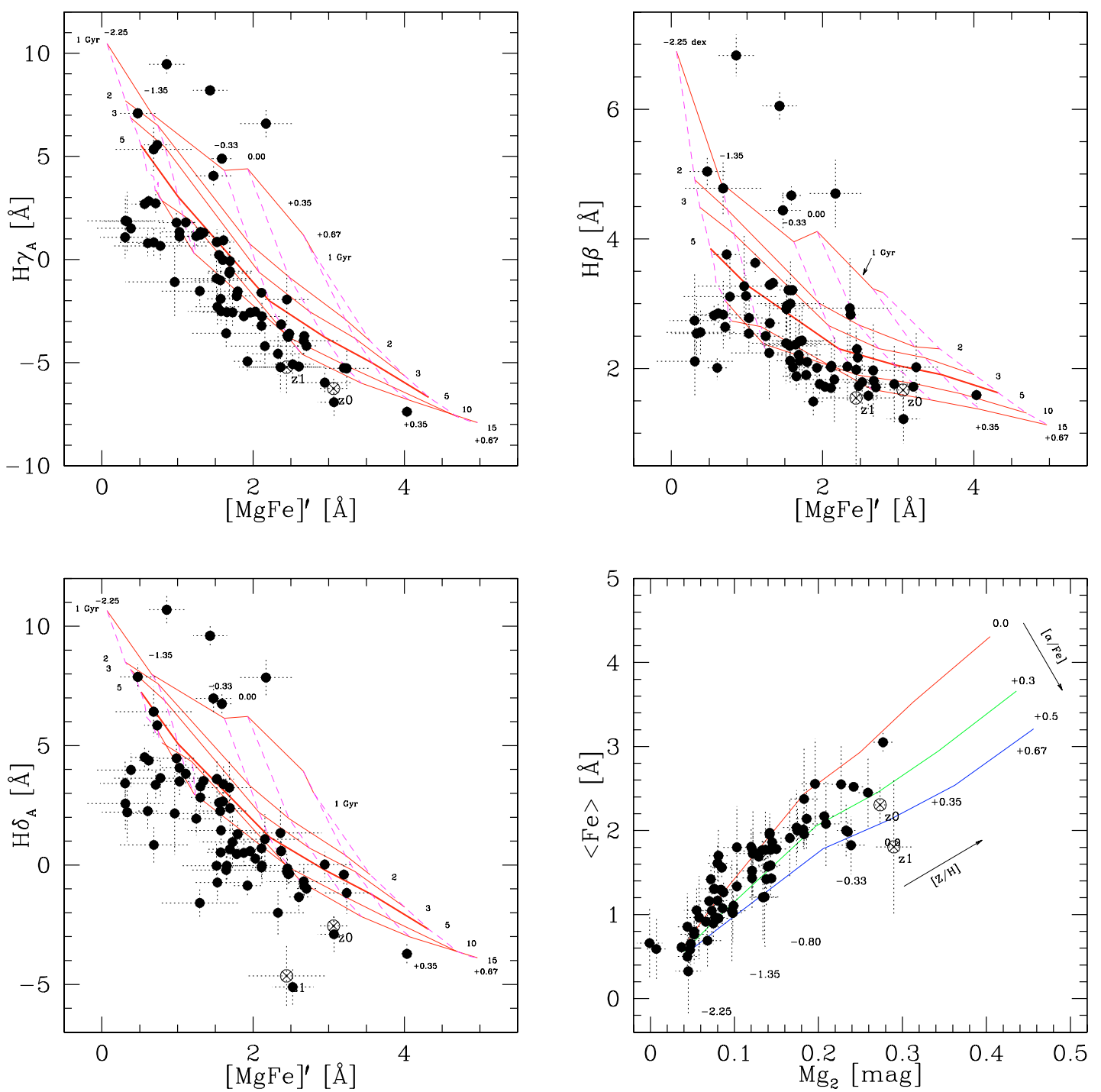

Fig. 6. Diagnostic Plots: $\mathrm{H} \gamma_{A}, \mathrm{H} \beta$, and $\mathrm{H} \delta_{A}$ vs. [MgFe]' age-metallicity diagnostic plot for stellar populations with $[\alpha / \mathrm{Fe}]=0.3$ dex. The lower right panel shows the $[\alpha / \mathrm{Fe}]$ diagnostic plot with $\mathrm{Mg}_{2}$ vs. $\langle\mathrm{Fe}\rangle$. Solid circles are M 31 globular clusters. SSP model predictions were taken from Thomas et al. $(2003,2004)$ and are shown in the age-metallicity grids as solid isochrones for stellar populations with ages 1, 2, 3, 5, 10, and $15 \mathrm{Gyr}$ and as dashed iso-metallicity tracks for metallicities $[\mathrm{Z} / \mathrm{H}]=-2.25,-1.35,-0.33,0.0,+0.35$, and +0.67 dex, for a stellar population with $[\alpha / \mathrm{Fe}]=0.3 \mathrm{dex}$. The SSP model in the lower right panel shows iso- $[\alpha / \mathrm{Fe}]$ tracks for metallicities $[\mathrm{Z} / \mathrm{H}]=-2.25$ to +0.67 and an age of 13 Gyr. Open circles mark M 31 bulge fields at different galactocentric radii, see Table 1. NB87, which has the highest metallicity among our sample globular clusters, is the only object with $[\mathrm{MgFe}]^{\prime} \approx 4 \AA$ and $\langle\mathrm{Fe}\rangle \approx 3 \AA$. It should be stressed again that the shown diagnostic grids are not suited to compare individual globular clusters, because of the $\alpha / \mathrm{Fe}$-sensitivity of Balmer indices. All grids have to be interpolated for each individual globular cluster according to its $[\alpha / \mathrm{Fe}]$. However, $\mathrm{H} \beta$ shows the weakest response to $\alpha / \mathrm{Fe}$ variations (see Fig. 4).

globular cluster sub-populations within a wide range of metallicities.

\section{Results}

\subsection{Ages and metallicities}

Figure 6 shows the M 31 globular cluster data, indicated by solid circles, together with theoretical SSP model predictions taken from Thomas et al. (2003, 2004). The model grid covers ages from 1 to $15 \mathrm{Gyr}$ and metallicities $[\mathrm{Z} / \mathrm{H}]$ from -2.25 to +0.67 dex, indicated by solid and dashed lines respectively.
The 5 Gyr isochrone is indicated as a thicker line to guide the eye when comparing diagnostic plots constructed with different Balmer-line indices. We note that direct comparisons of data and diagnostic grids are misleading when the $[\alpha / \mathrm{Fe}]$ abundance of each individual globular cluster is not taken into account (see previous section). However, to allow an assessment of the mean age and metallicity distribution of all globular clusters and to check for consistency of these predictions from all three diagnostic diagrams, we plot the data together with diagnostic grids for stellar populations with $[\alpha / \mathrm{Fe}]=0.3 \mathrm{dex}$.

The majority of our sample of M 31 globular clusters are consistent with old ages, However, the sample spans a wide 
range of ages, with $\sim 10 \%$ of the entire sample younger than 5 Gyr. Several globular clusters have index values which are consistent with formal ages similar or less than $\sim 1 \mathrm{Gyr}$ at an average metallicity $\langle[\mathrm{Z} / \mathrm{H}]\rangle \approx-0.4$ dex. Unfortunately, the models do not cover ages below $1 \mathrm{Gyr}$ and we do not attempt to extrapolate the grid, being aware of the potential uncertainties introduced by the AGB-phase transition which occurs at ages at or below $\sim 1$ Gyr. Depending on the total metallicity, the AGB can contribute $\sim 50 \%$ to the total bolometric light at these young ages (Renzini \& Buzzoni 1986; Ferraro et al. 1995). Theoretical models show that, although the fractional contribution of the AGB to the total light is subject to only small model-to-model fluctuations, the exact onset and duration of the AGB-phase transition depends on the treatment of classical mixing vs. overshooting (e.g. Alongi et al. 1993).

Clusters with such young ages are consistent with the findings of Beasley et al. (2004) who derive ages for their subsample of young globular clusters from a comparison with empirical spectra of young LMC globular clusters. This comparison is backed by their $\mathrm{H} \beta$ measurements being consistent with ages between 100 and $800 \mathrm{Myr}$ within the latest Bruzual \& Charlot (2003) SSP models 7 .

We find several M 31 globular clusters with near-solar metallicities and one object (NB87) reaching a formal $[\mathrm{Z} / \mathrm{H}] \approx$ $+0.5 \pm 0.1 \mathrm{dex}$ at a formally derived age of $9 \pm 2 \mathrm{Gyr}$, and a $[\alpha / \mathrm{Fe}]=0.09 \pm 0.05$. It is the most metal-rich globular cluster in our sample. Because of its relatively high metallicity, this object might host a stellar population representative of those in massive early-type galaxies, although it is slightly underabundant in $\alpha$-elements.

\subsubsection{Age-metallicity distributions and correlations}

In the following we compute mean ages and metallicities for our sample globular clusters using the information acquired from all three diagnostic grids. Based on the $\mathcal{R}$-parameter ranking and the sensitivity of Balmer lines to abundance variations (see Puzia et al. 2004a), we assign a weighting of 1.0 to values obtained from the $\mathrm{H} \gamma_{A}$ grid and a weighting factor of 0.5 to results obtained from the $\mathrm{H} \beta$ and $\mathrm{H} \delta_{A}$ grids. The reason for this weighting pattern are internal systematics in the age-metallicity diagnostic grids utilizing different Balmer-line indices (for details see Puzia et al. 2004a).

Using this averaging prescription, we construct age and metallicity histograms for the M 31 globular cluster sample, which are shown in Fig. 7. The upper left panel shows the age distribution of observed M 31 globular clusters. A clear peak around 10-12 Gyr dominates the age structure. The majority of the globular cluster sample $(\sim 60 \%)$ has formal ages older than 9 Gyr. A surprising feature of the age structure in the observed

\footnotetext{
${ }^{7}$ Note that the Bruzual \& Charlot (2003) models do not include predictions for stellar populations with a given $[\alpha / \mathrm{Fe}]$ ratio, contrary to the models of Thomas et al. $(2003,2004)$. However, given the young ages, the sensitivity of the $\mathrm{H} \beta$ index to $[\alpha / \mathrm{Fe}]$ variations is relatively weak, assuming the changes in chemical composition are correctly modeled. Hence, to first order the age-metallicity predictions of Beasley et al. (2004) should be relatively robust. This is not the case for higher-order Balmer Lick indices (Puzia et al. 2004b).
}
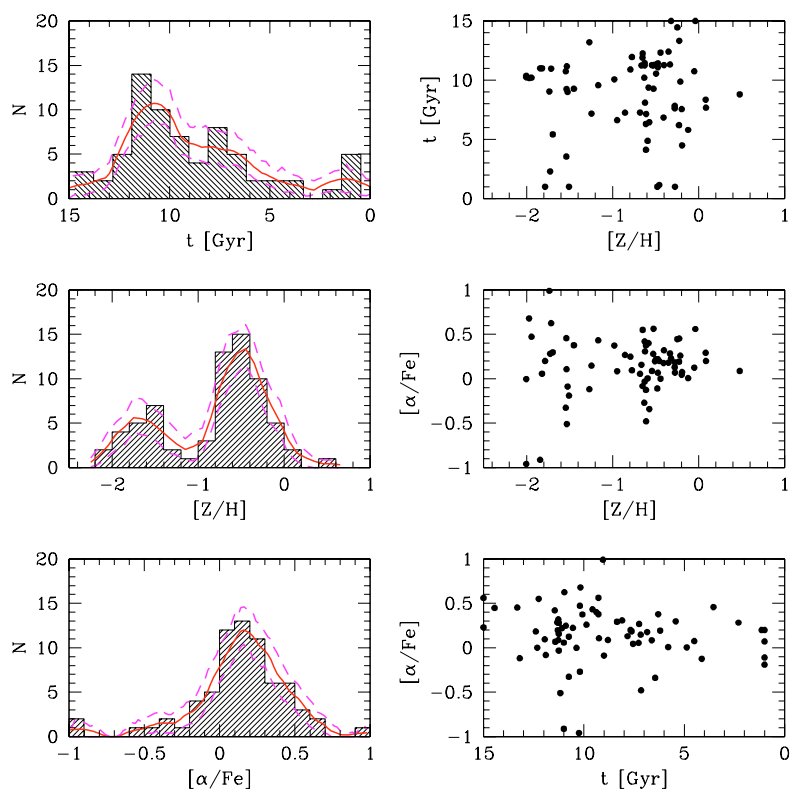

Fig. 7. Ages, metallicities, and $[\alpha / \mathrm{Fe}]$ ratios for M 31 globular clusters. Left panels show histograms for age, metallicity, and $[\alpha / \mathrm{Fe}]$ ratios for our sample globular clusters. Solid lines represent non-parametric probability density estimates using a variable-width Epanechnikov kernel (Silverman 1986) with their 90\% confidence limits (dashed lines). Right panels show correlations between the three parameters. Error bars are not shown for clarity; the mean error in age is $\Delta t / t \approx$ $1 / 3$, the mean uncertainty for metallicity and $[\alpha / \mathrm{Fe}]$ is $\sim 0.2$ dex.

M 31 globular cluster system is a population of intermediateage objects with formal ages between 6 and 8 Gyr. The mean uncertainty at those ages varies between 2 and 3.5 Gyr. In particular, this feature is not reproduced by the overlap of old iso-age tracks, which is the result of increasing flux from hot horizontal-branch stars with increasing age, at metallicities below $\sim-0.6$ dex. Only six globular clusters are affected by the region where the age determination is ambiguous. For these clusters the code randomly assigns either old or young age values.

After clipping globular clusters with ages $\leq 1 \mathrm{Gyr}$, a heteroscadastic KMM test ${ }^{8}$ (Ashman et al. 1994) shows that a bimodal age distribution is more likely than a single-peak Gaussian distribution with a mean at $\sim 11 \mathrm{Gyr}$. We find a $0.1 \%$ likelihood for the single-peak distribution and point out that a bimodal distribution is also more likely than a tri-modal fit. For the bimodal distribution the KMM test gives peaks at $t_{1}=10.9 \pm 0.3$ and $t_{2}=6.5 \pm 0.4 \mathrm{Gyr}^{9}$ with dispersions 1.7 and $3.5 \mathrm{Gyr}$, respectively. The integrity of the intermediate-age peak persists when we overplot a non-parametric probability

8 Two Gaussian modes with independent mean values and dispersion are fit to the data, as opposed to a homoscedastic fit where two modes with independent mean values but same dispersions are adopted during the fit.

9 The given errors are errors of the mean. Systematic errors are larger and of the order of 2-3 Gyr. For a homoscedastic KMM test the peak values change only marginally within the uncertainties. The mean dispersion of both peaks is $2.4 \mathrm{Gyr}$. 
density estimate with its $90 \%$ confidence limits. We use a nonparametric variable-width Epanechnikov kernel (Silverman 1986) that is consistent with the mean age uncertainty of each individual globular cluster.

In the left middle-row panel of Fig. 7 we present the metallicity distribution of studied M 31 globular clusters. The distribution is clearly bimodal stretching from around $-2.2 \mathrm{dex}$ to values slightly above solar. A heteroscedastic KMM-test gives peaks at $-1.66 \pm 0.05$ and $-0.45 \pm 0.04$ dex with dispersions 0.23 and 0.29 dex, respectively. From an empirical calibration of optical/near-IR colors with Milky Way globular clusters, Barmby et al. (2000) find peaks around $-1.4 \pm 0.05$ and $-0.6 \pm 0.05 \operatorname{dex}^{10}$. We note that our sample is biased towards metal-rich globular clusters which is a consequence of the sample selection. The latter was driven by the observational setup and focused mainly on M 31's disk and bulge (see Perrett et al. 2002; Beasley et al. 2004). Hence, the mean of each peak and its dispersion are subject to change for larger samples. In particular, the ratio between metal-poor and metal-rich globular clusters is not expected to be representative for the entire globular cluster system. This is at least partly the reason for the offset between our spectroscopic and Barmby et al.'s photometric mean metallicity in the metal-poor peak. However, it is less likely to explain the $\sim 3 \sigma$ offset for the metal-rich peak entirely, which might be a consequence of different absolute metallicity scales of the used SSP models.

In the upper right panel of Fig. 7 we present the agemetallicity correlation plot. We find old globular clusters at all metallicities. For globular clusters with formal ages below $\sim 8 \mathrm{Gyr}$, the evidence for metallicity bimodality remains. A group of five clusters (B315-038, B321-046, B322-049, B327-053, and B380-313) with ages below 5 Gyr and metallicities around -1.8 dex appears as a very distinct feature in the plot. The other metal-rich young objects are clustered around $[\mathrm{Z} / \mathrm{H}] \approx-0.6$ to -0.2 dex. If the group of five young metalpoor globular clusters is excluded, the overall age dispersion increases from $\sigma_{\mathrm{mp}} \approx 1.3 \mathrm{Gyr}$ for objects with metallicities below -1.0 dex to $\sigma_{\mathrm{mr}} \approx 3.4 \mathrm{Gyr}$ for metal-rich counterparts. The mean metallicity of intermediate-age globular clusters with ages between 3 and $8 \mathrm{Gyr}$ is $-0.61 \pm 0.11$ dex with a dispersion of 0.48 dex. Without two clusters from the young metal-poor population (B321-046 and B380-313), the mean changes to $-0.49 \pm 0.08$ dex and a dispersion 0.34 dex. There is tentative evidence that this relatively metal-rich intermediate-age globular cluster sub-population might have a bimodal metallicity distribution in itself with peaks around -0.6 and -0.2 dex. Such a multi-modality in the metallicity distribution of M 31 globular clusters was already discovered by Barmby et al. (2000) in their photometric study (see their Fig. 19). It is also worthy of notice that the metal-poor peak of this intermediate-age sub-sample coincides with the metal-rich peak of old globular clusters, while the metal-rich intermediate-age clusters appear to have $\sim 0.4$ dex higher mean metallicities than the average metal-rich old globular cluster. However, these results need follow-up analyses with larger samples.

$\overline{10}$ These metallicity values are on the Zinn-West scale, which is generally adopted throughout this work.
Irrespective of the completeness and metallicity coverage of our sample, we do find subpopulations of old ( 10-12 Gyr), intermediate-age ( $\sim 6-8 \mathrm{Gyr})$, and few young ( $\$ 2 \mathrm{Gyr})$ globular clusters in M 31. Our results are fully consistent with the recent spectro-photometric study of 172 globular clusters by Jiang et al. (2003), who find a significant population of intermediate-age and young globular clusters (see their Fig. 6). Their results also indicate that metal-rich globular clusters are on average younger than metal-poor globular clusters. It would be presumptious to speculate on the significance of this result ex parte, given our limited sample size (70 out of $460 \pm 70$ globular clusters). With the current dataset we cannot conclusively estimate the fractions of these new types of intermediate-age and young globular clusters and assess whether these sub-populations are small components or fundamental ingredients of the M 31 globular cluster system. In any case, the distribution of globular cluster ages suggests that M 31 managed to form star clusters that survived as globular clusters, until a few Gyr ago.

\subsection{2. $[\alpha / \mathrm{Fe}]$ ratios}

The determination of $[\alpha / \mathrm{Fe}]$ ratios yields very stable values within a few iterations of our grid-interpolation routine, which is the result of the small dependence of the $[\alpha / \mathrm{Fe}]$ diagnostic grid on age and metallicity. The left bottom-row panel of Fig. 7 shows the distribution of $[\alpha / \mathrm{Fe}]$ ratios, for which a single-peak distribution appears to be a good approximation. The mean of the single-peak distribution is $0.14 \pm 0.04 \mathrm{dex}^{11}$ with a dispersion $\sigma=0.32$ dex. However, using the KMM test we find that a two-Gaussian distribution is more likely $(99.4 \%)$ than the single-peak distribution. The test yields a relatively narrow peak at $[\alpha / \mathrm{Fe}]=0.20 \pm 0.02 \mathrm{dex}$ with a dispersion $\sigma=0.19$ dex, and a relatively broad peak at $-0.03 \pm 0.16$ with a factor $\sim 2.5$ larger dispersion of $\sigma=0.50 \mathrm{dex}$. The mixture of these modes is six to one, where the super-solar $[\alpha / \mathrm{Fe}]$ peak is more populated.

We can now correlate $[\alpha / \mathrm{Fe}]$ ratios of our sample globular clusters with their ages and metallicities. The right middle-row and bottom-row panels of Fig. 7 illustrate the metallicity vs. $[\alpha / \mathrm{Fe}]$ and age vs. $[\alpha / \mathrm{Fe}]$ plots. We find no correlation in the metallicity vs. $[\alpha / \mathrm{Fe}]$ plot. Metal-poor and metal-rich clusters have similar super-solar mean $[\alpha / \mathrm{Fe}]$ ratios within the uncertainty of $0.1-0.2$ dex. We find an increasing dispersion towards lower metallicities, which is mainly due to a decreasing $[\alpha / \mathrm{Fe}]$ resolution at lower $[\mathrm{Z} / \mathrm{H}]$. The sample of the five clusters with ages $\sim 1 \mathrm{Gyr}$ and metallicities around -1.6 dex shows super-solar $[\alpha / \mathrm{Fe}]$ ratio $+0.21 \pm 0.11$. Metal-rich $([\mathrm{Z} / \mathrm{H}] \geq$ -1.0 dex) globular clusters with ages below 8 Gyr show solartype $\alpha / \mathrm{Fe}$ ratios, i.e. $[\alpha / \mathrm{Fe}]=0.05 \pm 0.05 \mathrm{dex}$. Their old counterparts, on the other hand, have a higher mean $[\alpha / \mathrm{Fe}]=0.23 \pm$ 0.03 . Hence, we find evidence for an age- $[\alpha / \mathrm{Fe}]$ relation in the metal-rich globular cluster system.

An eye-catching feature of the age- $[\alpha / \mathrm{Fe}]$ correlation plot (right bottom-row panel of Fig. 7) is the decreasing scatter in $[\alpha / \mathrm{Fe}]$ with decreasing age. Globular clusters with ages

\footnotetext{
11 Median: 0.18 dex with 0.15 dex semi-interquartile range.
} 

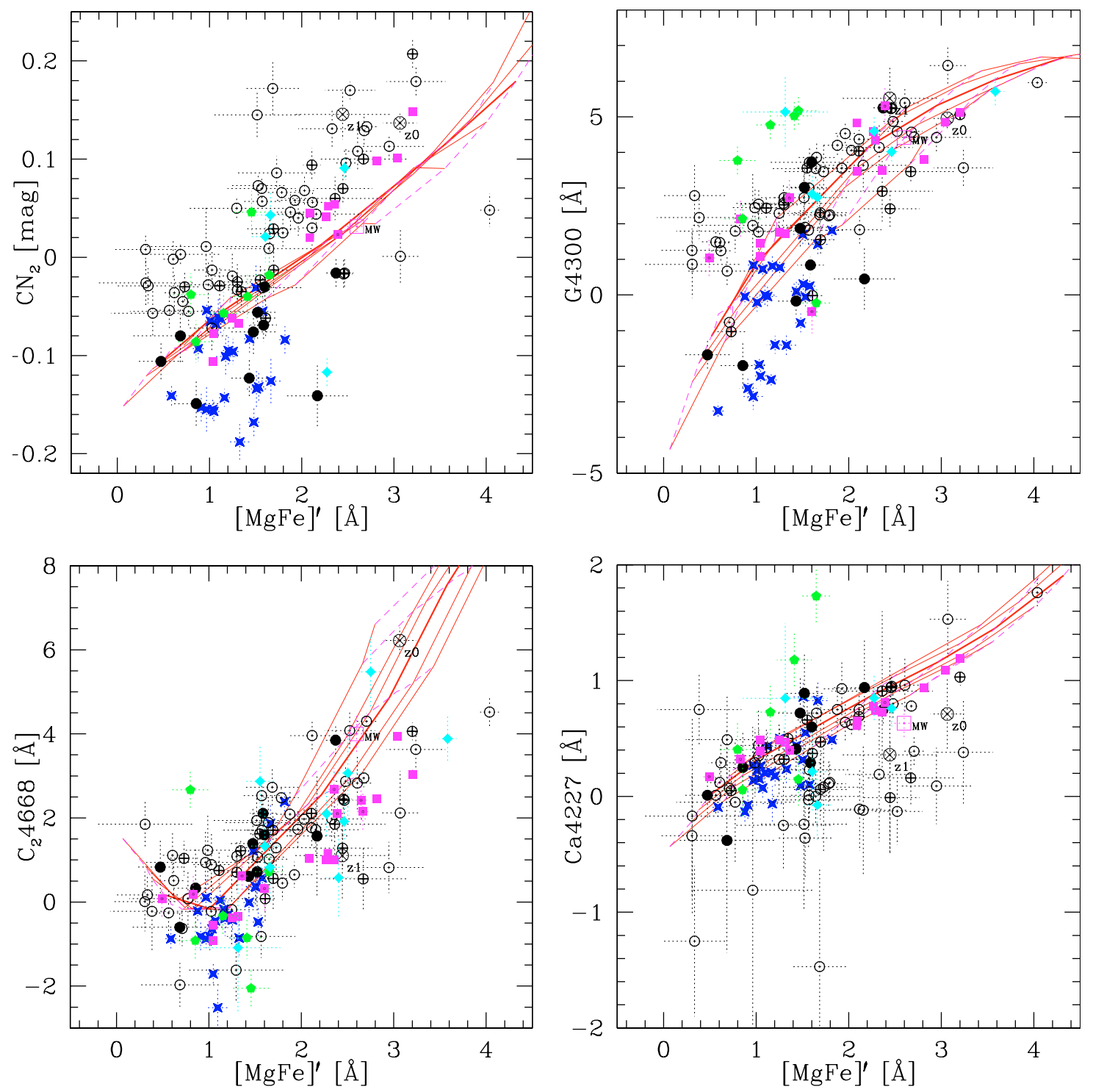

Fig. 8. $\mathrm{CN}_{2}, \mathrm{G} 4300, \mathrm{C}_{2} 4668$, and Ca4227 vs. [MgFe]' for M 31 (circles), Milky Way (squares), M 81 (diamonds), LMC (stars), and Sculptorgroup spiral galaxy globular clusters (pentagons). The individual datasets are described in Sect. 4.3. The M 31 symbols are parameterized by the globular cluster age: filled circles show globular clusters younger than $5 \mathrm{Gyr}$, circles with a central plus sign are intermediate-age clusters with ages between 5 and $8 \mathrm{Gyr}$, while open circles show globular clusters older than 8 Gyr. Note the offsets in CN and Ca4227 indices between old (open circles) and young (filled circles) M 31 globular clusters which is most likely a result of a factor three relative nitrogen enhancement of the old cluster system.

above 8 Gyr have a mean $[\alpha / \mathrm{Fe}]=0.18 \pm 0.05$ and a dispersion of $0.37 \mathrm{dex}^{12}$. Younger objects have a smaller $[\alpha / \mathrm{Fe}]$ ratio of $0.09 \pm 0.09$ and a dispersion of 0.21 dex. The sub-sample of globular clusters with ages $\$ 2 \mathrm{Gyr}$ is consistent with solar abundance ratios $[\alpha / \mathrm{Fe}]=0.03 \pm 0.08 \mathrm{dex}$ and a $\sigma=0.18 \mathrm{dex}$.

\section{2. $C N$ enhancement}

In the following section, we investigate correlations between ages, metallicities, and $[\alpha / \mathrm{Fe}]$ ratios and other Lick indices for our sample of M 31 globular clusters. Owing to previously observed CN anomalies of M 31 globular clusters (van den Bergh 1969; Burstein et al. 1984; Tripicco 1989; Davidge 1990a;

\footnotetext{
12 These values exclude two outliers at $[\alpha / \mathrm{Fe}]-1.0$ and $1.0 \mathrm{dex}$.
}

Brodie \& Huchra 1991; Beasley et al. 2004), we focus in the following analysis on correlations of the $\mathrm{CN}$ index with globular cluster age, metallicity, and $[\alpha / \mathrm{Fe}]$ ratio.

Since $\mathrm{CN}_{2}$ has its blue background passband designed to avoid the $\mathrm{H} \delta$ (see Fig. 3 in Puzia et al. 2004b) feature and has a calibration uncertainty of the transformation to the Lick system which is smaller than for $\mathrm{CN}_{1}$, we favor $\mathrm{CN}_{2}$ throughout the subsequent analysis and refer to it as the $\mathrm{CN}$ index. The $\mathrm{CN}$ index is a measure of the strength of the molecular $\mathrm{CN}$ band at $4150 \AA$.

In Fig. 8 we plot $\mathrm{CN}$ vs. $[\mathrm{MgFe}]^{\prime}$ along with SSP models for ages from 1 to $15 \mathrm{Gyr}$ (isochrones are solid lines), metallicities -2.25 to +0.67 dex (iso-metallicity tracks are dashed lines), and a $[\alpha / \mathrm{Fe}]$ ratio of 0.3 dex. The model grid is completely degenerate in age, metallicity, and $[\alpha / \mathrm{Fe}]$ and serves 
only as a reference line. In the plot we parameterize M 31 globular clusters by their age: open circles mark old clusters with formal ages $>8 \mathrm{Gyr}$, open circles with a central plus sign are intermediate-age globular clusters with ages between 5 and $8 \mathrm{Gyr}$, and solid circles show clusters formally younger than 5 Gyr. We find a clear offset in the $\mathrm{CN}-[\mathrm{MgFe}]^{\prime}$ sequence between old and young M 31 globular clusters. Young objects tend to have weak $\mathrm{CN}$ indices and fall below the model tracks, while old globular clusters show strong $\mathrm{CN}$ indices and populate the region above the model tracks. The $\mathrm{CN}$ index offset in the range $[\mathrm{MgFe}]^{\prime}=0-1.8 \AA([\mathrm{Z} / \mathrm{H}]$ between -2.3 and -0.6 dex) between these two groups is $0.13 \pm 0.02 \AA$. The intermediate-age group falls in between the young and old subpopulation. However, a $\Delta \mathrm{CN}=0.11 \pm 0.03$ with respect to young globular clusters makes it rather consistent with $\mathrm{CN}$ indices of old globular clusters. An interesting observation is the scarcity of globular clusters with small $\mathrm{CN}$ indices at $[\mathrm{MgFe}]>$ $2 \AA$. The mechanism which drives the $\mathrm{CN}$ difference between young and old globular clusters may be a function of metallicity. Unfortunately, our sample is still too small to explore any metallicity dependence.

\subsubsection{Is the CN-offset a consequence of age, metallicity, and/or $[\alpha / \mathrm{Fe}]$ differences?}

The $\mathrm{CN}$ index is mainly sensitive to the carbon and nitrogen abundance. In relatively cool giants that dominate the integrated light of old stellar populations, most of the carbon is locked in $\mathrm{CO}$, so that the nitrogen abundance affects the $\mathrm{CN}$ index more directly. Main-sequence dwarfs do not contribute to the $\mathrm{CN}$ absorption (Burstein et al. 1984), since molecules dissociate at higher $\log g$. Smaller $\mathrm{CN}$ absorption is also consistent with higher effective temperatures, which are expected in young and/or metal-poor stellar populations. The SSP models of Thomas et al. $(2003,2004)$ predict an offset of $\Delta \mathrm{CN} \approx$ 0.02-0.04 mag between $13 \mathrm{Gyr}$ and $3 \mathrm{Gyr}^{13}$ for stellar populations with metallicities between $[\mathrm{Z} / \mathrm{H}] \approx-1.35$ and 0.0 , and solar-type abundance ratios. Since the models were calculated for defined $[\alpha / \mathrm{Fe}]$ ratios, we can test the influence of $\alpha$-element enhancement on the $\mathrm{CN}$ index. The maximum $\mathrm{CN}$ index offset between stellar populations with $[\alpha / \mathrm{Fe}] 0.5$ and $0.0 \mathrm{dex}$, for ages up to $13 \mathrm{Gyr}$ and metallicities up to solar, is $\sim 0.02 \mathrm{mag}$. For super-solar metallicities the difference does not exceed $0.025 \mathrm{mag}$. Age and metallicity are therefore excluded as primary parameters that drive the $\mathrm{CN}$ offset.

Since we find evidence for an age- $[\alpha / \mathrm{Fe}]$ relation, we have reason to assume that the $\mathrm{CN}$ offset is produced by varying $[\alpha / \mathrm{Fe}]$ ratios. For an $[\alpha / \mathrm{Fe}]$ ratio of 0.2 dex the models predict very similar maximum $\mathrm{CN}$ offsets of $\sim 0.045$ mag between 3 and $13 \mathrm{Gyr}$ for solar and super-solar metallicites, still a factor 3 too small for the measured offset. The models predict smaller offsets for smaller metallicities. Going to highly supersolar $[\alpha / \mathrm{Fe}]$ ratios $\sim 0.5 \mathrm{dex}$, we find $\mathrm{CN}$ offsets of the order of 0.1 mag. However, such high $\alpha$-element enhancements are inconsistent with the measured $[\alpha / \mathrm{Fe}]$ ratios of the young

\footnotetext{
13 The mean age of the sub-population of globular clusters with ages below 5 Gyr is $2.5 \pm 1$ Gyr.
}

Table 3. Index response of the $\mathrm{CN}$ index to a factor three increase in nitrogen abundance. The values were calculated with model predictions taken from Thomas et al. (2003).

\begin{tabular}{lrrrrrr}
\hline \hline & \multicolumn{7}{c}{$\Delta \mathrm{CN}(3 \times \mathrm{N}-$ solar $)$} \\
{$[\alpha / \mathrm{Fe}]$} & 0.0 & 0.0 & 0.0 & +0.5 & +0.5 & +0.5 \\
{$[\mathrm{Z} / \mathrm{H}]$} & -1.35 & -0.33 & 0.00 & -1.35 & -0.33 & 0.00 \\
\hline $3 \mathrm{Gyr}$ & 0.023 & 0.050 & 0.068 & 0.023 & 0.051 & 0.069 \\
$13 \mathrm{Gyr}$ & 0.029 & 0.059 & 0.082 & 0.030 & 0.060 & 0.083 \\
\hline
\end{tabular}

sub-sample. In summary, any combination of differences in age, metallicity, and $[\alpha / \mathrm{Fe}]$ ratio between young and old M 31 globular clusters cannot explain the offset in their mean $\mathrm{CN}$ index.

\subsubsection{Is the $\mathrm{CN}$-offset a consequence of carbon and/or nitrogen abundance differences?}

Because Thomas et al. (2003) provide index predictions for stellar populations with an enhanced nitrogen abundance, we can compute a zeroth-order estimate of the increase in nitrogen abundance that is consistent with the observed $\mathrm{CN}$ index offset. Table 3 summarizes offsets in the $\mathrm{CN}$ index as a response to a factor three increased nitrogen abundance for different ages, metallicities, and $[\alpha / \mathrm{Fe}]$ ratios. Note that the abundances of other species remain unchanged. While other indices do not respond significantly, the increase in nitrogen abundance is expected to decrease the Ca4227 index (see Tripicco \& Bell 1995). Indeed, we observe such a decrease which is illustrated in Fig. 8. However, we refrain from a more quantitative analysis due to the poor internal calibration quality of this index (see Puzia et al. 2002b). For the mean age $\sim 13 \mathrm{Gyr}$, a metallicity $\sim-0.4 \mathrm{dex}$, and $[\alpha / \mathrm{Fe}]$ ratio $\sim 0.2 \mathrm{dex}$, we find a predicted $\mathrm{CN}$ offset of the order $\sim 0.07 \mathrm{mag}$, consistent with a factor three increase in nitrogen abundance. Together with the $\mathrm{CN}$ offset resulting from the age difference and under the assumption that the young globular cluster sub-population is not enhanced in nitrogen, the predicted $\mathrm{CN}$ offset amounts to $\sim 0.09-0.11 \mathrm{mag}$, which is in the right ballpark as the measured value. In fact, the nitrogen enhancement appears to be even larger than a factor of three.

Since the Lick system provides indices which are primarily sensitive to carbon abundance, we attempt to address the question of whether a variation in carbon abundance can explain the $\mathrm{CN}$-index offset between young and old M 31 globular clusters. For this purpose we plot $\mathrm{G} 4300$ and $\mathrm{C}_{2} 4668$ vs. $[\mathrm{MgFe}]^{\prime}$ in the middle and right panel of Fig. 8. G4300 measures the strength of the $G$-band which is dominated by $\mathrm{CH}-$ band absorption and a few $\mathrm{Fe}$ and $\mathrm{Cr}$ features; $\mathrm{C}_{2} 4668$, on the other hand, measures the $\mathrm{C}_{2}$ Swan band at $4700 \AA$, but its index passbands also include many weak $\mathrm{Fe}, \mathrm{Ti}, \mathrm{Cr}$, and $\mathrm{Sc}$ lines. Both indices are primarily sensitive to carbon abundance (Tripicco \& Bell 1995). To first order we do not see any significant offset between young and old globular clusters in the G4300 and $\mathrm{C}_{2} 4668$ index plot, although there are indications for differences in $G$-band strength at very low $[\mathrm{MgFe}]^{\prime}$ (i.e. metallicities). We therefore conclude that a difference in carbon abundance is not the driving factor for the observed $\mathrm{CN}$ offset. 
A more detailed look at the red background passband of the $\mathrm{CN}$ index reveals that the index is contaminated by two iron features at $\lambda \lambda 4260$ and $4272 \AA$ while the blue background passband has a weak Mn I feature located at its blue edge at $\lambda 4084 \AA$. Although this Mn feature is fairly weak, changes in $\mathrm{Mn}$ abundance might have a significant influence on the $\mathrm{CN}$ index. The blue background passband is relatively narrow $(12.5 \AA)$ and located $\sim 50 \AA$ away from the main $\mathrm{CN}$ feature, and small changes in Mn abundance can therefore translate in a large leverage of the pseudo-continuum flux inside the feature passband. Hence, complex, non-solar abundance ratio variations might influence the $\mathrm{CN}$ index strength, as well. However, a quantitative analysis of these effects is beyond the scope of this work as detailed modeling is not available at the time.

We conclude that the offset in $\mathrm{CN}$ index between young and old M 31 globular clusters is likely due to a nitrogen enhancement of old globular clusters by a factor three or more, compared to the young globular cluster system. Our results are consistent with the studies of Li \& Burstein (2003) and Burstein et al. (2004) who find a systematically higher nitrogen abundance in M 31 globular clusters as compared to Galactic counterparts $^{14}$.

\subsection{Comparison with globular clusters in other spiral and dwarf galaxies}

In this section we present a comparison of globular clusters in M 31, Milky Way, M 81 (Sa), LMC, and Sculptor-group spiral (Sa-b) galaxies. To avoid uncontrolled biases due to varying instrumental setups and spectral characteristics (i.e. resolution, sky-subtraction, flux-calibration, etc.), we strictly compare parameters derived from index measurements that were obtained in the same index system. For conformity with SSP model predictions we choose index passbands defined by Worthey et al. (1994) and for higher-order Balmer lines the passbands of Worthey \& Ottaviani (1997). The size of systematic uncertainties between different datasets is set by the calibration quality of the transformation to the Lick system. For this reason we collect only high-quality data from the literature with very good index calibrations to keep the systematics small. For the Milky Way sample we use data from Puzia et al. $(2002 b)^{15}$, and collect index measurements for LMC globular clusters from Beasley et al. (2002). M 81 globular cluster Lick index measurements were taken from Schroder et al. (2002). For a handful of globular clusters in the Sculptor-group spiral galaxies NGC 55, 247, 253, and 300, Olsen et al. (2004) measured Lick indices. A list of index measurements was kindly provided to us by Knut Olsen. Due to small sample sizes we merge the M 81

\footnotetext{
${ }^{14}$ We note that the metallicity coverage of their globular cluster sample is not consistent between M 31 and Galactic globular clusters. This point is discussed further below in the discussion section.

15 Although we are aware of the Cohen et al. (1998) data, we do not use these in the following analysis because of the lack of $\mathrm{H} \delta_{A}$ index measurements, which might introduce systematics in our analysis. However, the available index measurements are included in following diagnostic plots and marked as dotted squares. In general, they agree very well with the mean locus of the other globular cluster data taken from Puzia et al. (2002b).
}

and Sculptor-group globular clusters into one sample with ten globular clusters in total. We warn however that the individual globular cluster samples can have very different age, metallicity, and abundance ratio distributions. Diagnostic diagrams for all globular clusters are shown in Figs. 8 and 9.

\subsubsection{Ages and metallicities}

Figure 9 shows M 31, Milky Way, M 81, Sculptor-group spiral galaxy, and LMC globular clusters in three different age-metallicity diagnostic plots, as circles, squares, diamonds, pentagons, and stars, respectively. After extracting ages, metallicities, and $[\alpha / \mathrm{Fe}]$ ratios for globular clusters in other galaxies in exactly the same way as described earlier for M 31, we find several interesting systematic differences between the observed globular cluster systems. Age, metallicity, and $[\alpha / \mathrm{Fe}]$ distributions are shown in Fig. 10. Both the M 31 and Milky Way globular cluster systems are dominated by objects reaching oldest ages at $\sim 11-13$ Gyr. In addition to old clusters, the M 31 system hosts populations of intermediate-age (6-8 Gyr) and young $(\$ 2 \mathrm{Gyr})$ globular clusters, which are not observed in the Milky Way sample.

The mean age of the Milky Way sample is $12.0 \pm 1.3 \mathrm{Gyr}^{16}$ which is in good agreement with previous CMD results (11.5 \pm $1.3 \mathrm{Gyr}$, Chaboyer et al. 1998). The metallicities covered by M 31 and Milky Way globular clusters are similar, which again underlines the importance of careful sample selection when different globular cluster systems are compared (see Sect. 2.4). The metallicity distribution of the Milky Way sample is bimodal with peaks around -1.2 and -0.3 dex, although these estimates are uncertain due to the small sample size of each peak.

LMC globular clusters span a wide range in age from objects as young as $\sim 1 \mathrm{Gyr}$ to old clusters resembling ages of old Galactic counterparts. This is consistent with previous findings (see also Beasley et al. 2002, and references therein). The metallicity distribution of the LMC sample is consistent with a broad single peak with a mean $[\mathrm{Z} / \mathrm{H}]=-0.95 \pm 0.09 \mathrm{dex}^{17}$ and a dispersion $\sigma=0.42$ dex.

We combine the samples of M 81 and Sculptor-group globular clusters because of the small number of studied objects in each galaxy. The combined age distribution shows peaks around 12 and 8 Gyr with a clear indication for intermediateage globular clusters, which is similar to the M 31 rather than the Milky Way globular cluster age distribution. However, very young objects are missing in this sample. The metallicity distribution is peaked around -0.6 dex with only one cluster at roughly solar metallicity and one metal-poor candidate at -1.5 dex. We warn that the distributions are subject to change once larger and more representative samples are available.

Although the samples of observed globular clusters in all galaxies are still small, all cover the brightest fraction of each

\footnotetext{
16 This uncertainty is the formal statistical uncertainty derived from our approximation routine, while the systematic uncertainty derived from the comparison of results from different diagnostic plots is $\sim 3.5$ Gyr. The median value is $12.1 \mathrm{Gyr}$.

17 The median of the distribution is -0.89 dex.
} 

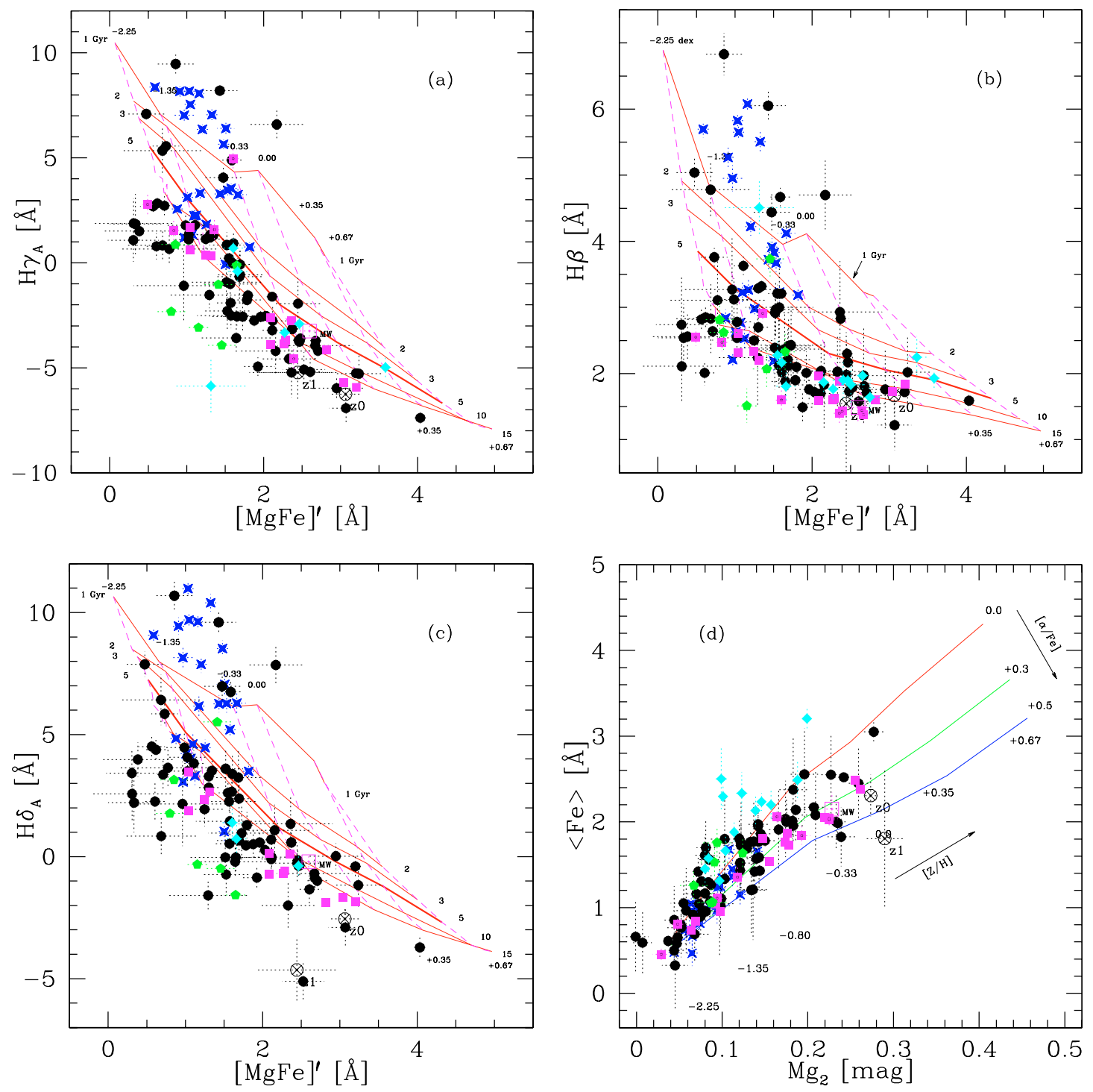

Fig. 9. Comparison of age-metallicity diagnostic diagrams panels a-c) for M 31 (solid circles), M 81 (diamonds), Milky Way (solid squares), and LMC globular clusters (stars), as well as globular clusters in Sculptor-group spiral galaxies (pentagons). The panels show diagnostic plots using different Balmer indices, $\mathrm{H} \gamma_{A}, \mathrm{H} \beta$, and $\mathrm{H} \delta_{A}$. Also shown is the $\alpha / \mathrm{Fe}$ diagnostic grid panel d). Milky Way globular clusters are from Puzia et al. (2002b), while LMC globular clusters were taken from Beasley et al. (2002). Squares with a central dot indicate measurements of Galactic globular clusters performed by Cohen et al. (1998) and calibrated by Beasley et al. (2004). Lick indices for M 81 globular clusters were performed by Schroder et al. (2002), and Olsen et al. (2004) measured indices for globular clusters in Sculptor-group spiral galaxies. Note that $\mathrm{H} \delta_{A}$ measurements are not available in the Cohen et al. dataset. Most of the M 81 data have no higher-order Balmer index measurements. Also plotted are index measurements for the nuclear diffuse light of the Milky Way and M 31. A large open square marks the index measurements for the Galactic Bulge light in Baade's Window, taken from Puzia et al. (2002b), and two crossed open circles indicate measurements for the diffuse light of the M 31 bulge at two different galactocentric radii (labeled as $z 0$ and $z 1$, see Table 1). Note, that all data and models use the same index passband system. SSP model predictions are as in Fig. 6.

globular cluster system. Keeping this in mind, we can draw the following conclusions. The age structure of each globular cluster system suggests that luminous globular clusters experienced significantly different formation histories in all three galaxies. While the question about the presence of intermediate-age globular clusters in the Milky Way will certainly be answered by our ongoing spectroscopic survey of Local Group globular clusters, photometric CMD studies suggest that a small fraction of metal-rich Galactic globular clusters might be younger than $~ 9$ Gyr (Salaris \& Weiss 2002). However, among the studied bright clusters we find no Galactic counterparts for the intermediate-age and/or young globular clusters found in M 31, M 81, LMC, and Sculptor-group spiral galaxies. From Fig. 10 it seems that the age structure of the M 31 cluster system is a composite of globular clusters in all other studied galaxies. This aspect might suggest that M 31 globular clusters with ages $\lesssim 9$ Gyr were accreted from satellite galaxies.

\subsection{2. $[\alpha / \mathrm{Fe}]$ ratios}

The average $[\alpha / \mathrm{Fe}]$ ratios of LMC and Milky Way globular clusters are $\sim 0.1-0.2$ dex higher than the mean $\alpha$-element 


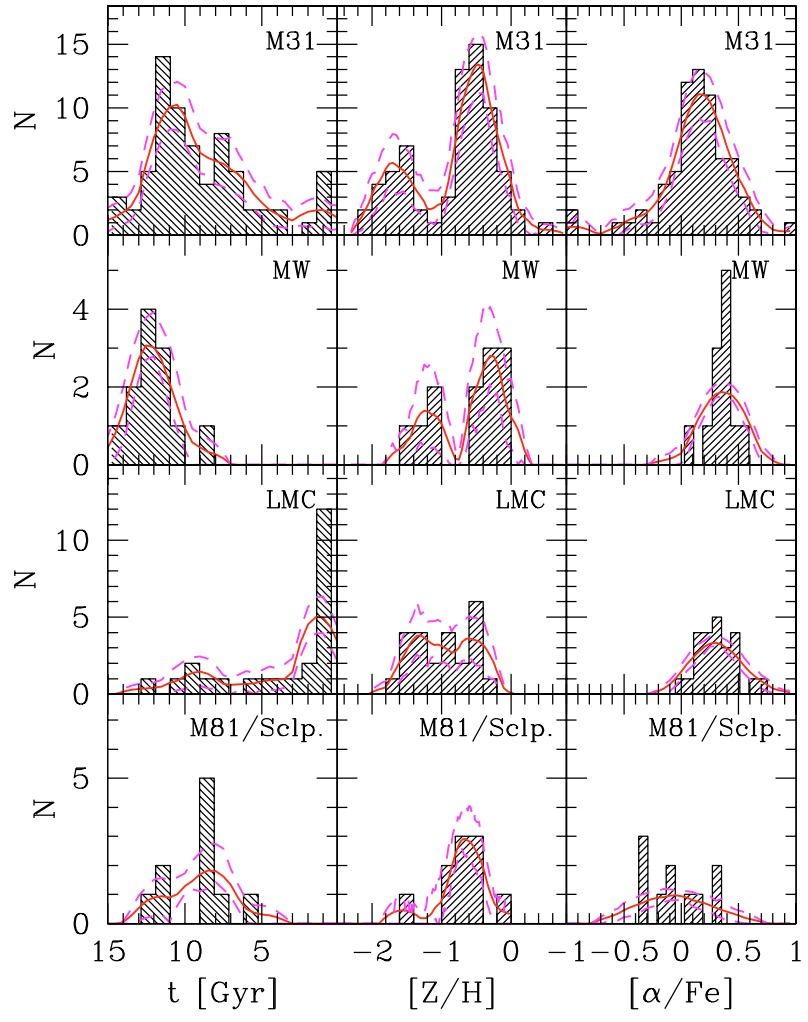

Fig. 10. Age, metallicity, and $[\alpha / \mathrm{Fe}]$ distributions for globular clusters in M 31, Milky Way, LMC, M 81, and Sculptor-group spiral galaxies. Due to small sample sizes, the M 81 and Sculptor-group samples were merged. Solid lines are non-parametric probability density estimates with their $90 \%$ bootstraped confidence limits shown as dashed lines (see Silverman 1986, for details).

enhancement of M 31 globular clusters (see also Fig. 9d). The mean $\alpha$-element enhancement of the Galactic sample is clearly super-solar with a mean $0.35 \pm 0.04 \mathrm{dex}^{18}$ and a dispersion $\sigma=0.13 \mathrm{dex}$. The mean $[\alpha / \mathrm{Fe}]$ ratio of the $\mathrm{LMC}$ cluster sample is $0.32 \pm 0.04$ dex and has a dispersion of 0.17 dex. The combined $\mathrm{M} 81 /$ Sculptor sample has a mean $[\alpha / \mathrm{Fe}]=$ $-0.05 \pm 0.08$ and a fairly broad distribution with a dispersion of $\sigma=0.25$ dex.

Yet, all three globular cluster systems show super-solar $[\alpha / \mathrm{Fe}]$ ratios, which implies enrichment dominated by type-II supernovae (e.g. Matteucci 1994). Super-solar mean $\alpha$-element enhancements around $\sim 0.2-0.35$ dex are observed in highresolution spectra of single stars in Galactic halo and bulge globular clusters (Barbuy et al. 1999; Cohen et al. 1999; Carretta et al. 2001, 2004; Origlia et al. 2002; Origlia \& Rich 2004). This is in good agreement with the values derived here and can be considered as a zero-point calibration of our $[\alpha / \mathrm{Fe}]$ scale (see also Maraston et al. 2003; Thomas et al. 2003, 2004). However, there are Galactic globular clusters, such as Pal 12, which show peculiar abundance signatures with roughly solar $[\alpha / \mathrm{Fe}]$ ratios (Cohen 2004). Judging from their kinematics, these objects appear to be stripped from nearby satellite galaxies.

\footnotetext{
18 The median value is $0.37 \mathrm{dex}$.
}

Compared to the mean $[\alpha / \mathrm{Fe}]$ of LMC clusters, the mean $[\alpha / \mathrm{Fe}]$ ratio of intermediate-age M 31 globular clusters is $\sim 0.1-0.2$ dex lower, and suggests that accretion of LMC-type globular cluster systems to build up the intermediate-age globular cluster population in M 31 is unlikely. This low $[\alpha / \mathrm{Fe}]$ is rather consistent with the mean value found for the M 81/Sculptor cluster sample. It is also consistent with the abundance pattern in thin/thick disk stars in the Milky Way (e.g. Edvardsson et al. 1993; Fuhrmann 1998).

\subsubsection{Carbon and nitrogen abundance}

Beginning with the study of Burstein et al. (1984), globular clusters in M 31 were found to be unique in individual line indices compared to their Galactic counterparts. Enhanced CN-band absorption in M 31 clusters was later found to be due to nitrogen enhancement (Ponder et al. 1998; Li \& Burstein 2003; Burstein et al. 2004). How do the CN indices of M 31 globular clusters compare to other globular cluster systems? In Fig. 8 we plot all other globular clusters together with M 31 globular clusters, parameterized by their age.

Old M 31 globular clusters appear on average $\mathrm{CN}$-enhanced compared to the other globular cluster systems. Intermediateage clusters, on the other hand, compare well in their mean $\mathrm{CN}$ index with the other cluster systems. We find a striking coincidence in $\mathrm{CN}$ strength between LMC and young M 31 globular clusters. The youngest M 31 clusters populate the same locus in the $\mathrm{CN}$ vs. $[\mathrm{MgFe}]^{\prime}$ plot as most LMC globular clusters. Compared to SSP models, which predict CN strength for solar-type abundance pattern, this suggests that both LMC and young M 31 globular clusters are underabundant in carbon and/or nitrogen. Since no systematic difference in the $\mathrm{C}_{2} 4668$ and G4300 indices, which are most sensitive to carbon abundance, is found between LMC and young M 31 globular clusters, the $\mathrm{CN}$ offset suggest a difference in nitrogen abundance. Consistent with this picture is the systematic Ca4227 offset between old M 31 globular clusters, on the one hand, and LMC and young M 31 globular clusters, on the other hand (see Sect. 4.2).

\subsection{Kinematics}

In a recent radial-velocity study of the M 31 globular cluster system, Morrison et al. (2004) found a significant subpopulation of globular clusters showing thin-disk kinematics with metallicities down to -2.0 dex. The presence of such a distinct disk globular cluster sub-system with low-metallicity members implies an early formation epoch of M 31's thin disk. We correlate the kinematics of Morrison et al. with our ages, metallicities, and $[\alpha / \mathrm{Fe}]$ ratios. A cut in normalized residual radial velocity at $|0.75| \mathrm{km} \mathrm{s}^{-1}$ relative to the thin-disk model of Morrison et al. (2004) selects globular clusters which are likely members of the thin-disk population, as suggested by the authors. This selection leaves 18 objects with thin-disk kinematics in the sample.

This sub-sample covers ages between one and $11.9 \mathrm{Gyr}$. It has a mean age $8.1 \pm 0.9 \mathrm{Gyr}$ and a dispersion $\sigma=3.6 \mathrm{Gyr}$. 
The average metallicity is $[\mathrm{Z} / \mathrm{H}]=-0.78 \pm 0.16$ dex with $\sigma=0.68 \mathrm{dex} ;$ its mean $[\alpha / \mathrm{Fe}]$ is slightly super-solar at $+0.12 \pm$ 0.07 dex with a sample dispersion of 0.30 dex. We confirm that thin-disk globular clusters span a wide range in metallicity from about -1.8 dex up to solar values, as reported by Morrison et al. (2004).

If globular clusters with residuals smaller than $|0.25| \mathrm{km} \mathrm{s}^{-1}$ are selected, six objects remain in the sample and all but one ${ }^{19}$ have intermediate to young ages $\lesssim 8$ Gyr. Beasley et al. (2004) found also three young globular clusters with clear thin-disk kinematics. Their median metallicity decreases to $-1.02 \pm$ 0.31 dex with a higher metallicity dispersion of 0.75 dex and their median $[\alpha / \mathrm{Fe}]$ ratio increases to $0.26 \pm 0.05 \operatorname{dex}(\sigma=$ 0.12 dex). This suggests that M 31 globular clusters with clear thin-disk kinematics are members of the intermediate-age cluster sub-population. Although their metallicities are inconsistent with typical abundances found in the Milky Way's thin-disk stellar population, their $[\alpha / \mathrm{Fe}]$ ratios are in accord with those of Galactic bulge globular clusters (see Sect. 4.3.2). It would be interesting to obtain a larger sample of thin-disk globular clusters in M 31 (1) to test the maximum age of this globular cluster sub-population, and (2) to check whether or not their chemical abundances are in contrast with disk/bulge globular clusters in the Milky Way.

In Fig. 11 we plot the residual radial velocity, which was calculated relative to the thin disk model of Morrison et al. as a function of globular cluster age, metallicity, and $[\alpha / \mathrm{Fe}]$ ratio. There is a clear bimodality in the residuum distribution and we split the sample into clusters that fall in the hatched region which indicates globular clusters with normalized disk-model residuals $\leq|20| \mathrm{km} \mathrm{s}^{-1}$, and globular clusters with larger residuals indicating halo membership. We consider the sub-population with small residuals as a thin/thick disk component, being aware of potential contamination by halo objects. About $70 \%$ of our globular cluster sample falls into the thin/thick disk category, excluding only halo members. Based on the number counts of the halo and thin/thickdisk sample and assuming a Gaussian distribution of velocities around the mean of the thin-disk model with dispersions 30 and $150 \mathrm{~km} \mathrm{~s}^{-1}$, for the disk and halo population, respectively, we expect $2 \pm 2$ halo interlopers in our thin/thick-disk sample and less than one genuine disk globular cluster in the halo sample.

The most striking feature in the upper panel of Fig. 11 is that most globular clusters of the halo population have ages older than 9 Gyr, with a median age of 10.4 Gyr. Only two halo globular clusters have intermediate ages and we find one extremely young cluster (B324-S51) that is inconsistent with disk kinematics. The thin/thick-disk population, on the other hand, has a median age of 9.4 Gyr. The halo sub-sample shows a clearly bimodal distribution of metallicities with peaks around -1.6 and -0.5 dex.

It is interesting that the velocity dispersion around the Morrison et al. model in the thin/thick-disk sample decreases towards younger globular clusters; from $|0.47| \mathrm{km} \mathrm{s}^{-1}$ for

19 This cluster (B171) has a formal age of $10.8 \mathrm{Gyr}$ and might be a halo interloper.

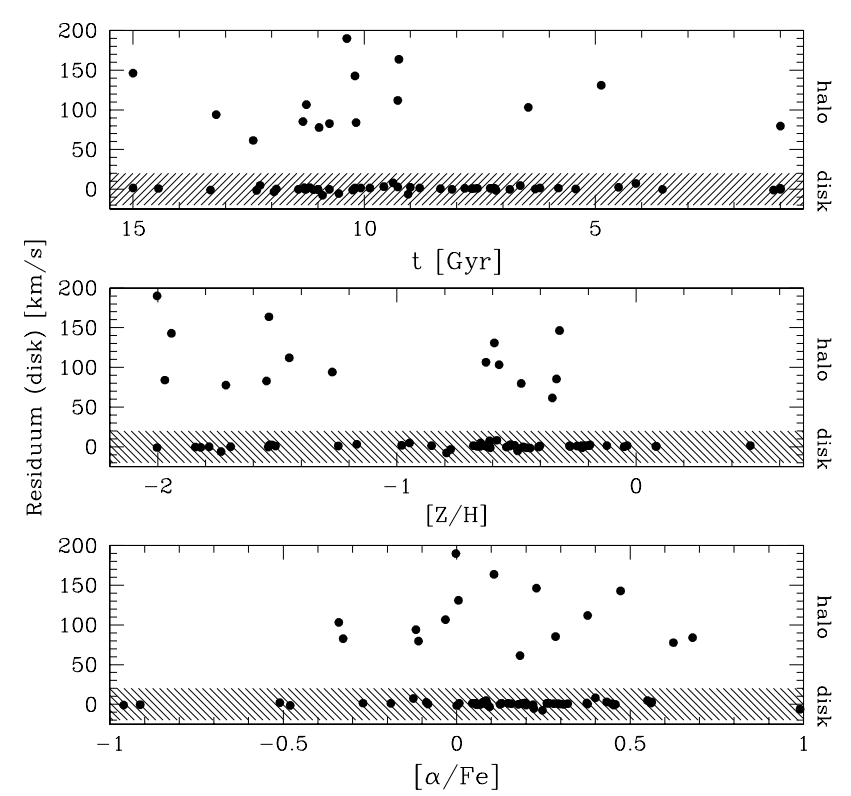

Fig. 11. Residual radial velocities relative to a disk model of Morrison et al. (2004) as a function of age, metallicity, and $[\alpha / \mathrm{Fe}]$ ratio for M 31 globular clusters. Error bars are not shown for clarity; the mean error in age is $\Delta t / t \approx 1 / 3$, the mean uncertainty in metallicity and $[\alpha / \mathrm{Fe}]$ is $\sim 0.2$ dex. The typical error in radial velocity is $\leq 12 \mathrm{~km} \mathrm{~s}^{-1}$.

objects with ages $>8 \mathrm{Gyr}$ to $0.38 \mathrm{~km} \mathrm{~s}^{-1}$ for globular clusters younger than $8 \mathrm{Gyr}$.

The distribution of $[\alpha / \mathrm{Fe}]$ ratios for halo and thin/thick-disk globular clusters is shown in the lower panel of Fig. 11. We find an average $[\alpha / \mathrm{Fe}]=0.17 \pm 0.07 \mathrm{dex}$ with a dispersion of $0.33 \mathrm{dex}$, for the halo sample. The thin/thick-disk sample has a similar mean $[\alpha / \mathrm{Fe}]$ at $0.14 \pm 0.01 \mathrm{dex}$ and a dispersion of 0.32 dex.

We find a mild age- $[\alpha / \mathrm{Fe}]$ correlation in the thin/thick-disk sample of the form $[\alpha / \mathrm{Fe}]=(0.013 \pm 0.011) \cdot t+(0.076 \pm 0.122)$, with a rms of 0.12 dex for a weighted least-square fit, where $t$ is given in Gyr. We compute weights for individual globular clusters from an average of upper and lower $1 \sigma$ uncertainties. Normally, these errors are asymmetric due to the skewness of diagnostic grids. No significant correlation between $[\alpha / \mathrm{Fe}]$ and metallicity is found for thin/thick-disk globular clusters.

\subsection{Structural parameters}

In the following section we study the correlations between globular cluster core radi, $R_{\mathrm{c}}$, half-light radii, $R_{\mathrm{hl}}$, tidal radii, $R_{\mathrm{t}}$ (see King 1962), and the projected galactocentric distance, $r_{\text {proj }}$ with previously derived ages, metallicities, and $[\alpha / \mathrm{Fe}]$ ratios. We collect data on structural parameters for M 31 globular clusters from Barmby \& Huchra (2001), for Milky Way globular clusters from the 2003 update of the McMaster catalog ${ }^{20}$ (Harris 1996), and for LMC clusters from Mackey \& Gilmore (2003).

Since for extragalactic globular clusters only projected galactocentric distances are available, we need to project the 3D positions of the Galactic globular cluster system on

\footnotetext{
20 http://physun. physics.mcmaster.ca/ harris/mwgc.dat
} 


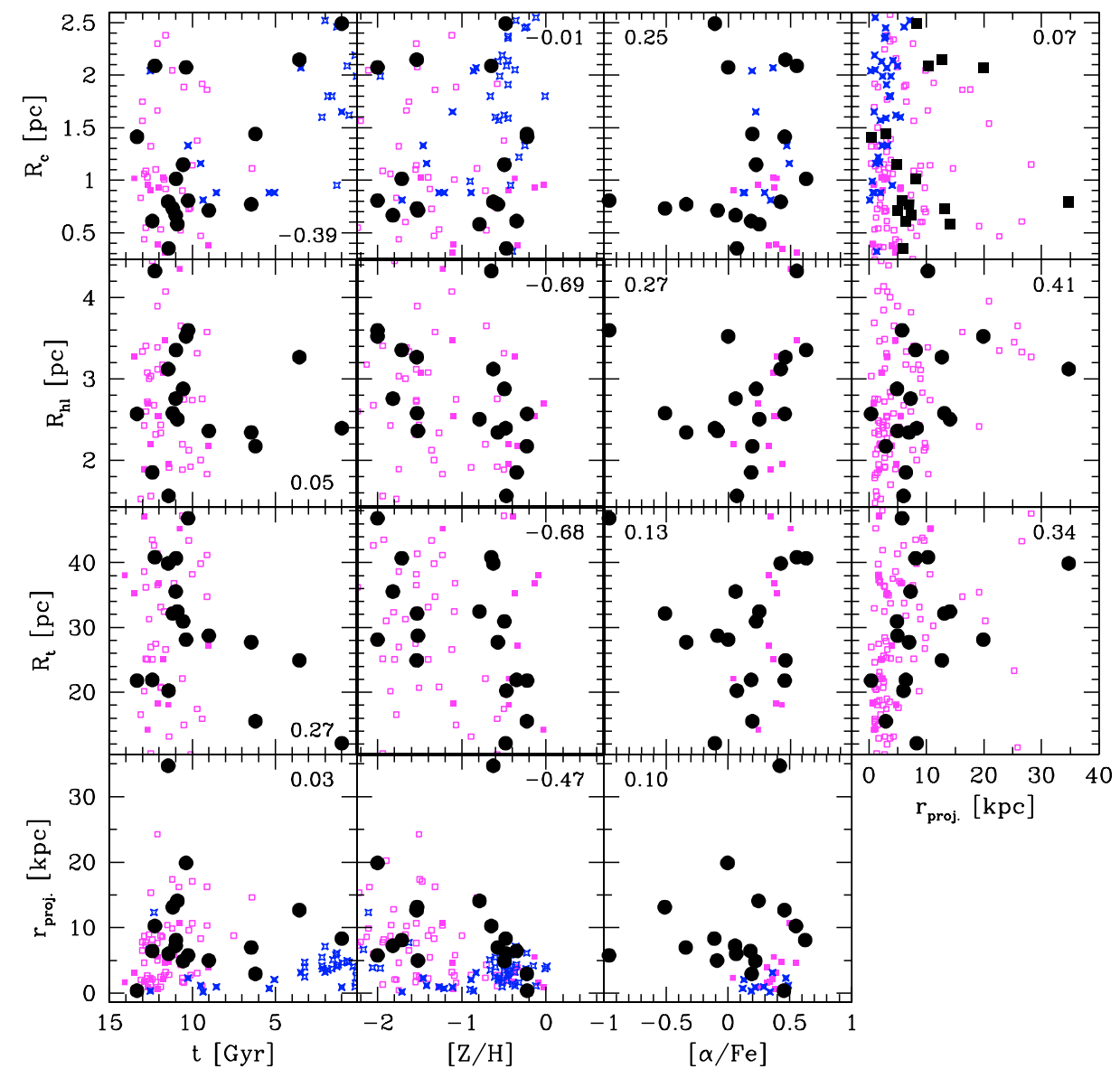

Fig. 12. Structural parameters, $R_{\mathrm{c}}$ (core radius), $R_{\mathrm{hl}}$ (half-light radius), and $R_{\mathrm{t}}$ (tidal radius), as a function of age, metallicity, and $[\alpha / \mathrm{Fe}]$ ratio for globular clusters in M 31 (circles), Milky Way (squares), and LMC (stars). Data on structural parameters were taken from Barmby \& Huchra (2001) for M 31 globular clusters, from the 2003 update of the McMaster catalog (Harris 1996) for Milky Way globular clusters, and from Mackey \& Gilmore (2003) for LMC clusters. Information on age and metallicity for Galactic globular clusters is parameterized by their photometric (open squares, data taken from Salaris \& Weiss 2002) and spectroscopic origin (solid squares, data taken from Puzia et al. $2002 \mathrm{~b}$ ). The structural parameters are shown as a function of projected galactocentric radius, $r_{\text {proj }}$, in the right-column panels. The bottom row of panels shows the projected galactocentric radius as a function of age, metallicity, and $[\alpha / \mathrm{Fe}]$ ratio. In a corner of each panel the Spearman rank correlation coefficient is given.

a 2D surface on the sky for a fair comparison between Galactic and extragalactic cluster systems. Fortunately, the McMaster catalog provides the 3D positions of individual Galactic globular clusters in the reference system of the Sun, so that the conversion to a projected galactocentric distance is easy. We simulate the view towards the Milky Way globular cluster system similar to that towards M 31, by applying a similar pitch angle of $77^{\circ}$ for the Milky Way disk and the entire globular cluster system and randomly choosing a line of sight tilted with respect to the Earth-Galactic center axis.

In Fig. 12 we compare structural parameters with globular cluster ages, metallicities, and $[\alpha / \mathrm{Fe}]$ ratios. We also plot the structural parameters as a function of projected galactocentric radius in the very right column of Fig. 12. For each sub-panel we compute the Spearman rank correlation coefficient for the M 31 globular cluster sample. The number is given in a corner of each panel (1 indicates perfect correlation, -1 anti-correlation).

In general, the parameter space coverage between the three globular cluster systems is in good agreement. We find correlations in the $R_{\mathrm{hl}}$ and $R_{\mathrm{t}}$ vs. $[\mathrm{Z} / \mathrm{H}]$ panels, where the Spearman correlation coefficient is around -0.7 with a statistical significance for a correlation of $>99.7 \%$ in both cases. Since both the half-light radius $R_{\mathrm{hl}}$ and the tidal radius $R_{\mathrm{t}}$ appear to be weakly correlated with galactocentric radius $r_{\text {proj }}$, at least part of their correlation with metallicity might be due to a correlation of metallicity with galactocentric radius (reflecting the galactocentric distance dichotomy between metal-rich disk and metal-poor halo globular clusters; see Chernoff \& Djorgovski 1989; van den Bergh 1991; Côté 1999). This is consistent with the correlation in the $r_{\text {proj }}$ vs. $[\mathrm{Z} / \mathrm{H}]$ plot in Fig. 12. However, the sample selection might produce spurious correlations and it is important to check these relations with larger samples, in particular because the Galactic sample does not show such a strong correlation of $R_{\mathrm{hl}}$ and $R_{\mathrm{t}}$ with metallicity.

We point out that similar size differences between metalpoor and metal-rich globular clusters were found in other galaxies (Kundu \& Whitmore 1998; Puzia et al. 1999; Larsen et al. 2001). A possible explanation for this difference might be the combined effects of mass segregation and the dependence 
of main-sequence lifetimes on metallicity, under the assumption of similar distribution of central potentials and half-mass radii (Jordán 2004). However, the size difference might be also entirely driven by projection effects which are due to a systematic difference in dynamical evolution of globular clusters on different orbits (Larsen \& Brodie 2003). If true, size differences should disappear at large galactocentric radii, where dynamical effects similarly affect metal-poor and metal-rich globular clusters. Intriguingly, Perrett (2001, Ph.D. Thesis) found an increasing size of metal-rich globular clusters with galactocentric radius, approaching the mean size of metal-poor globular clusters at large galactocentric distances. No galactocentric distance-size relation was found in the metal-poor globular cluster sub-population. Size information on more metalrich globular clusters at large galactocentric radii is needed to establish whether the size difference between metal-poor and metal-rich globular clusters is due to a dynamical effect or reflects differences in globular cluster formation.

It is well known that the globular cluster populations of M 31, LMC, and the Milky Way fall on the same "fundamental plane", which for globular clusters is defined by velocity dispersion, surface brightness, and radius (Djorgovski 1995). Together with the similar correlations between the structural parameters of globular clusters in M 31, Milky Way and LMC with their ages, metallicites, and abundance ratios, this suggests that the formation and evolution must have been similar for the studied clusters.

\subsection{Comparison with the integrated bulge light of M 31 and the Milky Way}

Early speculations by Baade (1944) claimed that the stellar populations in Galactic globular clusters would resemble those in the Milky Way bulge and the nucleus of M 31. This simplistic picture was revised soon after, when a more complete census of stellar populations in Galactic globular clusters was available. Only the most metal-rich globular clusters appear to host stellar populations similar to those in spiral bulges and early-type galaxies (e.g. Maraston et al. 2003).

In the following section we compare the integrated-light spectra of the M 31 nucleus, obtained in this study (see Sect. 2), the Milky Way bulge (taken from Puzia et al. 2002b), and metal-rich globular cluster populations of both galaxies. Figure 6 shows the location of the two M 31 bulge spectra (as crossed open circles) in age/metallicity and $[\alpha / \mathrm{Fe}]$ diagnostic grids for the two different galactocentric radii (see Fig. 1 and Table 1). Using the same technique as for the globular cluster spectra we derive luminosity-weighted ages, metallicities, and $[\alpha / \mathrm{Fe}]$ ratios. The values are summarized in Table 4 . Since the inner M 31 field and the Milky Way field were taken at similar mean galactocentric radii $(\sim 0.6 \mathrm{kpc})$, their stellar populations can be directly compared, assuming that no significant differences in population gradients are present between the two galaxies.

Given the statistical and systematic errors, the derived mean ages, metallicities, and $[\alpha / \mathrm{Fe}]$ ratios suggest that the stellar populations in both spiral bulges are similar. Both bulges
Table 4. Ages, metallicities, and $[\alpha / \mathrm{Fe}]$ ratios of the integrated light for three fields in the bulge of M 31 and the Milky Way. The spectra of all fields were taken at different median galactocentric distances, $r_{\text {median }}$, given in the second column. All measurements were performed in the same way as for the globular cluster data and are given with their statistical $1 \sigma$ uncertainties.

\begin{tabular}{lcccc}
\hline \hline Galaxy & $r_{\text {median }}[\mathrm{kpc}]$ & Age $[\mathrm{Gyr}]$ & {$[\mathrm{Z} / \mathrm{H}]$} & {$[\alpha / \mathrm{Fe}]$} \\
\hline MW & 0.55 & $9.4 \pm 1.4$ & $-0.22 \pm 0.06$ & $0.35 \pm 0.07$ \\
M 31 $(z 0)$ & 0.60 & $14.5 \pm 1.3$ & $-0.06 \pm 0.04$ & $0.38 \pm 0.07$ \\
M 31 $(z 1)$ & 2.48 & $14.9 \pm 2.2$ & $-0.22 \pm 0.12$ & $0.65 \pm 0.22$ \\
\hline
\end{tabular}

harbor old stellar populations, with metallicities close to solar values. There is a tendency for the M 31 bulge to have a slightly higher metallicity. Both stellar populations have clearly supersolar $\alpha / \mathrm{Fe}$ ratios, although the outer M 31 field seems to have a higher $[\alpha / \mathrm{Fe}]$ value.

There is no systematic difference in $\mathrm{CN}$ index between metal-rich old M 31 globular clusters, which are enhanced in nitrogen (see Sect. 4.2), and the corresponding M 31 bulge fields (see Fig. 8). However, compared to metal-rich Galactic globular clusters and the Galactic bulge, the $\mathrm{CN}$ index of the M 31 bulge is offset by $\sim 0.04$ and $\sim 0.1 \mathrm{mag}$, respectively, and suggest an enhacement in carbon and/or nitrogen abundance. The $\mathrm{C}_{2} 4668$ index for the central M 31 field (z0) is significantly higher with respect to the mean M 31 and Galactic globular clusters at similar $[\mathrm{MgFe}]^{\prime}$. This is not the case for the outer field ( $z 1)$. However, this $\mathrm{C}_{2} 4668$ offset is less pronounced when the central M 31 field is compared to the Milky Way bulge. In combination with the similar G4300 indices for metal-rich globular clusters and the bulges of M 31 (both sectors) and the Milky Way, this suggests that the central M 31 field $(z 0)$ is enhanced in carbon and nitrogen, compared to the Galactic bulge and metal-rich Galactic globular clusters. The outer M 31 field is likely to be enhanced in nitrogen only. The reduced Ca4227 indices of both M 31 fields with respect to metal-rich Milky Way globular clusters and Galactic bulge underline these results. Furthermore, we find an increase in $\mathrm{CN}$ index with increasing galactocentric radius for the $\mathrm{M} 31$ bulge. This suggests an increasing nitrogen enhancement, which in the case of the field stellar population in M 31 increases with radius. Such differences were already reported by Morgan \& Mayall (1957) who discovered that the integrated spectrum of M 31's nucleus is dominated by cyanogen absorption features. Recently, radial $\mathrm{NH}$ gradients in the M 31 bulge were found by Davidge et al. (1990).

In summary, the bulge of M 31 appears to be metal-rich, $\alpha$-element enhanced and old, similar to the Milky Way bulge. However, compared to the Galactic stellar populations there are significant differences in chemical composition. We find indications for an enhancement in carbon and nitrogen in the central region of M 31, while the integrated light of the outer stellar populations is consistent with a nitrogen enhancement only. It is well known that stellar populations in early-type galaxies show super-solar metallicities and various abundance ratios (Worthey et al. 1992; Sánchez-Blázquez et al. 2003). In this respect, our comparison shows that metal-rich M 31 globular 
clusters and early-type galaxies are more alike than stellar populations in early-type galaxies and metal-rich Galactic globular clusters.

\section{Discussion}

\subsection{Stellar streams and merging}

The existence of the recently discovered thin-disk population of M 31 globular clusters (Morrison et al. 2004) is a strong argument for an undisturbed disk evolution. Depending on the exact kinematical definition of the thin-disk population, we find that most, but not all, members of the thin-disk globular cluster population have ages younger than $\sim 10 \mathrm{Gyr}$ and metallicities $-2.0 \lesssim[\mathrm{Z} / \mathrm{H}] \lessgtr 0.0$ (see Sect. 4.4). The presence of old thindisk globular clusters suggests an early formation and smooth kinematical evolution of the thin-disk globular-cluster population. In particular, it implies in-situ formed star clusters. It would be therefore of great interest to determine the maximum age in a larger dataset of thin-disk globular clusters and to test how early M 31 's thin disk formed.

Recent very deep HST observations, pointed towards the halo of M 31, made the age and metallicity distributions of the targeted stellar populations directly accessible. These CMD studies revealed a dominant metal-rich stellar population with a surprisingly high metallicity, $[\mathrm{Z} / \mathrm{H}] \approx-0.6$ (Holland et al. 1996; Sarajedini \& Van Duyne 2001; Bellazzini et al. 2003; Rich et al. 2004, see also Fig. 1) and intermediate ages $\sim 6-8$ Gyr (Brown et al. 2003). There are indications for an even more metal-rich stellar population $([\mathrm{Z} / \mathrm{H}] \gtrsim-0.2)$ which is irregularly distributed throughout M 31's halo (Sarajedini \& Van Duyne 2001; Bellazzini et al. 2003). The case of a metalrich intermediate-age stellar population in the halo of a spiral galaxy is generally attributed to an accretion or merging event that led to the incorporation of external stellar populations and/or to infall of fresh material which fueled new star formation. Depending on the exact merging kinematics, tidally disrupted satellites remain in the halo for several Gyr, until their central regions eventually spiral inwards as a result of dynamical friction. Support for this picture comes from observations of significant large-scale sub-structure in the form of stellar streams in the halo of M 31 (Ibata et al. 2001, 2004; Ferguson et al. 2002; McConnachie et al. 2003, 2004). Other disk-dominated galaxies show similar indications of halo substructure, pointing to a hierarchical assembly of these systems (e.g. Zibetti et al. 2004).

Since accretion events tend in general to heat stellar disks, a good indicator for a puffed-up disk population is the ratio of disk scale length, $d$, to scale height $z_{0}$. A minor merger with a mass fraction of $0.05-0.2$ is expected to agitate the stellar disk and increase its vertical scale height by a factor $1.5-2$, compared to an undisturbed evolving disk (Velazquez \& White 1999). This is confirmed by observations of edge-on spirals with and without signs of interaction (Schwarzkopf \& Dettmar 2000,2001 ), where interacting spirals show $d / z_{0} \leq 7$. Largearea star counts in M 31 find that the scale height of the disk varies between 50 and $400 \mathrm{pc}$, while the scale length is $\sim 5-7 \mathrm{kpc}$ (Hopper 1995). The resulting $d / z_{0} \approx 12.5-140$ is placing the M 31 disk well within the regime of undisturbed stellar disks, implying a very small past merging rate. If, however, the mass accretion is smooth enough and/or occurs on preferentially tangential orbits so that the thin-disk integrity is unaffected, thin-disk evolution and mass accretion might be two spatially uncorrelated, but temporally coexisting processes (e.g. Font et al. 2001).

It is not clear if some M 31 globular clusters are associated with the two stellar streams discovered by Ibata et al. (2001) and McConnachie et al. (2004), because of the still too sparse kinematic information. However, Perrett et al. (2003) find kinematically coupled groups in the M 31 globular cluster system. Although the location of some globular clusters coincides with the general locus of the streams (see the northern stream in Fig. 1), it is unlikely that accretion contributed significantly to the assembly of the M 31 globular cluster system, in particular the thin-disk sub-population.

Morrison et al. (2004) find a velocity dispersion in the thindisk globular cluster system of $\sim 20-40 \mathrm{~km} \mathrm{~s}^{-1}$. Globular cluster systems in dwarf galaxies have typical velocity dispersions $30-80 \mathrm{~km} \mathrm{~s}^{-1}$ (e.g. Olsen et al. 2004). In general, the thin-disk globular clusters in M 31 have on average too small a velocity dispersion to be accreted from merging satellite galaxies, although the very unlikely case of a merging trajectory which is perfectly aligned with the disk plane could be a way to preserve a small velocity dispersion of the infalling cluster system during the accretion event.

Using the numbers from Ibata et al. (2001), the most massive stream is estimated to carry $10^{8}$ to $10^{9} M_{\odot}$ with a total $M_{V}=-14$ mag. Assuming an average specific frequency $S_{N} \approx$ 10 for dwarf galaxies of this luminosity (Durrell et al. 1996), the estimated number of accreted globular clusters is $\sim 4$. The other stream (McConnachie et al. 2004) carries less mass and will therefore contribute even less globular clusters. However, if both streams are associated with the two satellites M 32 and NGC 205 (both with a total luminosity $M_{V} \approx-16.4 \mathrm{mag}$ ), the contributed number of clusters might be as large as $20-40$ objects per galaxy, given typical specific frequencies $S_{N} \approx 2-8$ for these systems. M 32 hosts no globular clusters (Ashman \& Zepf 1998), although at least $\sim 20$ are expected.

If the two massive stellar streams added some globular clusters to M 31, it is unlikely that they are now part of M 31's thindisk globular cluster population, based on their trajectories. Both stellar streams have very radial orbits and therefore do not interact significantly with the thin-disk globular-cluster population (Ibata et al. 2004). Despite their potential past interaction(s), the evolution of a kinematically cool thin-disk globularcluster population could have remained undisturbed.

\subsection{Spatial distribution}

We find spatially clumped globular cluster sub-populations in M 31. Intermediate-age globular clusters preferentially reside inside $\sim 5 \mathrm{kpc}$ galactocentric distance, which is suggestive of a slightly younger inner-disk/bulge cluster population. Clusters with ages below $5 \mathrm{Gyr}$ are predominantly found at large radii along the major axis. Most of these globular clusters show thindisk kinematics and have metallicities below $-0.8 \mathrm{dex}$ (see also 
Fig. 11). Their projected positions (see Fig. 1) coincide with luminous star-forming regions in M 31's outer-disk, that were recently observed by GALEX ${ }^{21}$.

This spatial clustering suggests that halo, inner-disk/bulge, and thin-disk globular cluster sub-populations experienced different formation and/or evolution histories. While the intermediate-age population could have formed from previously enriched material several Gyr after the old halo population was formed, the young cluster population might be the offspring of star-formation triggered by a recent infall of fresh material in the outskirts of M 31. However, a direct connection between these globular cluster sub-populations and the two giant stellar streams in the halo is rather unlikely, although we find two young metal-rich young globular clusters (B337-G68 and B324-S51) close in projection to NGC 205. Since there is much more sub-structure visible in M 31's halo than the two reported stellar streams (e.g. Ferguson et al. 2002; McConnachie et al. 2004), direct connections between other stellar streams and globular cluster sub-samples are conceivable. Only a careful kinematic analysis of the stellar streams can clarify how much accretion contributed to the formation of M 31's halo and disk globular cluster system. Such ambitious surveys are on the way (see e.g. Ibata et al. 2004).

\subsection{Chemical enrichment}

In the following we discuss chemical characteristics of $M 31$ globular clusters that might be informative of their formation and evolution. While type-II supernovae predominantly eject $\alpha$-elements on very short timescales ( $\$ 10 \mathrm{Myr}$ ), the ejecta of type-Ia supernovae enrich the interstellar medium with $\mathrm{Fe}$ peak elements with a delay of typically 1 Gyr (Greggio 1997; Matteucci \& Recchi 2001; but see also Tsujimoto 2004, for a short-living type-Ia progenitor). These supernova types mark the two extremes of injection timescales for processed material back into the interstellar medium. Other elements such as carbon and nitrogen are predominantly produced by massive stars with a strong dependence on metallicity, on timescales 10-100 Myr (e.g. Maeder 1992). In general, the relative delay in progenitor lifetimes between type-II and Ia supernovae implies that abundance ratios can be used as tools to clock starformation timescales.

A detailed model of the chemo-dynamical evolution of globular cluster systems is not available yet and a comparison of our data with theoretical predictions is not possible (but see $\mathrm{Li}$ et al. 2004). However, our data on $[\alpha / \mathrm{Fe}]$ ratios and nitrogen enhancement of M 31 globular clusters allow us to make some qualitative statements on their formation timescales.

\subsection{1. $\alpha$-elements}

Super-solar $[\alpha / \mathrm{Fe}]$ ratios of M 31 globular clusters can be interpreted as the result of relatively short formation periods, which ended before a significant number of type-Ia supernovae could contribute to chemical enrichment of their parent gas clouds.

\footnotetext{
${ }^{21}$ http://www.galex.caltech.edu/popups/ gallery-M31.html
}

As chemical enrichment increases with time it requires successively higher star-formation rates to increase the $\alpha$-element enhancement to a certain level and "override" the chemical signature of previous enrichment episodes. Hence, the average trend of $[\alpha / \mathrm{Fe}]$ is expected to be a declining function of metallicity, as enough time will be given to type-Ia supernovae to lower this ratio, unless previous star-formation events were truncated before type-Ia supernovae started to eject large amounts of ironpeak elements. In the same line, a decreasing mean $[\alpha / \mathrm{Fe}]$ is expected at younger stellar-population ages.

We find no general correlation between $[\alpha / \mathrm{Fe}]$ and metallicity for our M 31 globular cluster sample, which indicates that the stellar populations in most of these objects were predominantly enriched by core-collapse (type-II) supernovae. In combination with the rather weak anti-correlation of age vs. $[\alpha / \mathrm{Fe}]$ and age vs. metallicity, this suggests that old metal-poor globular clusters formed from material which experienced a truncated enrichment, dominated by type-II supernova ejecta. Metal-rich, intermediate-age and young M 31 globular clusters, on the other hand, formed from gas that received its metals from type-II and Ia supernovae, perhaps during the simmering star-formation processes in $\mathrm{M}$ 31's disk. This indicates that stellar populations in the M 31 disk experienced significant enrichment by type-Ia supernovae. The dominant fraction of M 31 globular clusters, however, was enriched by type-II supernovae.

\subsubsection{Nitrogen enhancement}

Nitrogen is produced in two modes. One speaks of a primary and secondary nitrogen component, depending on whether the seed elements carbon and oxygen are synthesized from helium by the same star (primary) or were already in place when the star formed (secondary). Sources of primary nitrogen are massive, zero-metallicity fast rotating stars that suffer a hypernova explosion at the end of their very short lifetimes (typically a few Myr: Woosley \& Weaver 1982; Fryer et al. 2001; Heger et al. 2003). More massive and faster rotating hypernova progenitors generally inject more nitrogen into the interstellar medium (Heger et al. 2000). However, it is noted by Heger et al. that even a non-rotating $60 M_{\odot}$ star with a shallow entropy gradient can produce strong over-abundances of nitrogen. Secondary nitrogen is mainly ejected by strong stellar winds of intermediate-mass stars $\left(\sim 4-7 M_{\odot}\right)$ during their AGB phase (e.g. van den Hoek \& Groenewegen 1997). In general, stars with successively higher mass and higher angular momentum produce larger amounts of both $\alpha$-elements and nitrogen (e.g. Woosley \& Weaver 1995).

This implies that hypernova ejecta have both super-solar $[\alpha / \mathrm{Fe}]$ ratios and enhanced $\mathrm{N} / \mathrm{C}$ and $\mathrm{N} / \mathrm{O}$ ratios, as the production of primary nitrogen is based on the consumption of the seed elements carbon and oxygen. Moreover, $[\alpha / \mathrm{Fe}]$, and $\mathrm{N} / \mathrm{C}$ and N/O ratios should be positively correlated. This is different for AGB-star yields. van den Hoek \& Groenewegen (1997) show that the N/C ratio is expected to increase slightly as a function of stellar mass and metallicity, while N/O is predicted to have a much steeper correlation. Hence, over Gyrs of 

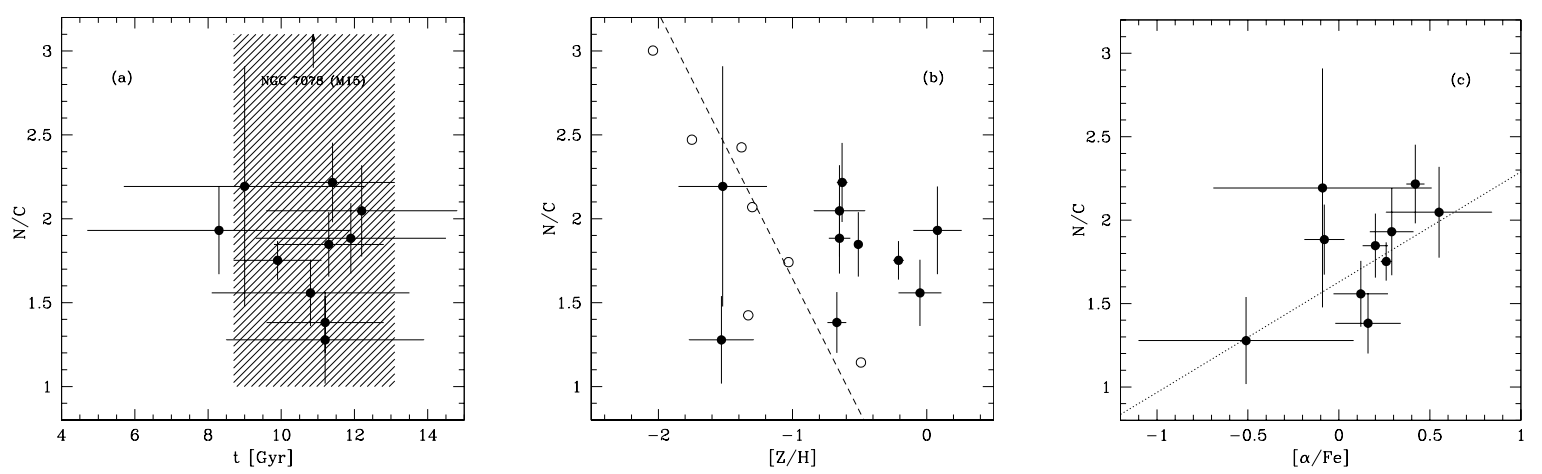

Fig. 13. Correlation between the $\mathrm{N} / \mathrm{C}$ ratio, computed as the ratio of the $\mathrm{NH}$ index and the $\mathrm{CH}(\mathrm{G} 4300)$ index vs. age, metallicity, and $[\alpha / \mathrm{Fe}]$ ratio. M 31 globular clusters are indicated by solid dots. Open circles mark Milky Way globular clusters. The hatched region in panel a) shows the typical locus where Galactic globular clusters reside. A linear least-square fit in panel b) to Galactic globular-cluster data is illustrated by a dashed line. The dashed line in panel c) shows a linear fit to M 31 globular clusters.

chemical evolution, we expect asymptotically decreasing $\mathrm{N} / \mathrm{C}$ and $\mathrm{N} / \mathrm{O}$ ratios as a function of increasing metallicity.

The importance of primary and secondary nitrogen enrichment is currently the subject of a lively debate in the literature. The need for a primary plateau-enrichment by zero-metallicity massive stars was raised by the detection of highly super-solar $[\mathrm{N} / \mathrm{Fe}]$ ratios in extremely metal-poor $([\mathrm{Fe} / \mathrm{H}] \lessgtr-4)$ Galactic halo stars (e.g. Norris et al. 2001, 2002; Christlieb et al. 2002, 2004) and metal-poor damped Lyman- $\alpha$ systems (e.g. Pettini et al. 2002).

To study N/C as a function of age, metallicity, and $[\alpha / \mathrm{Fe}]$, we match our observations with those of Burstein et al. (2004) and find ten matched M 31 globular clusters for which NH measurements are available. None of the Milky Way globular clusters for which $\mathrm{NH}$ measurements were performed by Burstein et al. have matched age, metallicity, and $[\alpha / \mathrm{Fe}]$ ratios derived from Lick indices in this work. We therefore substitute the latter with ages and metallicities derived from color-magnitude diagrams taken from Salaris \& Weiss (2002) and De Angeli et al. (2004, private communication). CMD metallicities are on the Zinn-West metallicity scale and were corrected for an assumed average $\alpha$-element enhancement of $[\alpha / \mathrm{Fe}]=+0.3 \mathrm{dex}$, using $[\mathrm{Z} / \mathrm{H}]=[\mathrm{Fe} / \mathrm{H}]-0.8 \cdot[\alpha / \mathrm{Fe}]$ (Trager et al. 2000). No $[\alpha / \mathrm{Fe}]$ determinations for the matched Galactic globular clusters are available to us at the moment, so that we have to postpone a comparison of $\mathrm{NH}$ and $[\alpha / \mathrm{Fe}]$ ratios between Milky Way and M 31 globular clusters.

The Galactic and M 31 globular cluster samples of Burstein et al. (2004) cover very different metallicity ranges (see panel b in Fig. 13). While most M 31 globular clusters fall in the range $-1 \lesssim[\mathrm{Z} / \mathrm{H}] \lesssim 0$, most of the Milky Way globular clusters have metallicities below -1.0 dex. Hence, a direct comparison of NH abundances between M 31 and Milky Way globular clusters becomes less meaningful. To bypass this drawback we focus on abundance ratios instead. Because of their virtually identical dissociation energies (Tomkin \& Lambert 1984), the strength of the near-UV NH band and the optical CH band around $4300 \AA$ can be used to derive the nitrogen to carbon $(\mathrm{N} / \mathrm{C})$ abundance ratio fairly accurately. The strength of the optical CH-band is measured by the G4300 Lick index which is available for all matched M 31 globular clusters as well as Milky Way globular clusters. We therefore refer to the $\mathrm{NH} / \mathrm{CH}(\mathrm{G} 4300)$ index ratio in the following as the $\mathrm{N} / \mathrm{C}$ ratio.

Figure 13 shows the N/C ratio as a function of globular cluster age, metallicity, and $[\alpha / \mathrm{Fe}]$. We find no clear correlation of N/C vs. age. Since the age resolution in the covered age range $(\sim 8-13 \mathrm{Gyr})$ is poor, it would be very interesting to obtain more NH data for intermediate-age and young M 31 globular clusters. We note that a correlation of increasing nitrogenenhancement with older age is observed in starburst galaxies (Esteban \& Peimbert 1995). If nitrogen-enhancement is mainly produced by hypernovae, older globular clusters are expected to show on average higher $\mathrm{N} / \mathrm{C}_{\text {ratios }}{ }^{22}$.

We find enhanced N/C ratios in metal-rich M 31 globular clusters compared to their Galactic counterparts at similar metallicities (see panel b in Fig. 13). Almost all M 31 clusters with $[\mathrm{Z} / \mathrm{H}] \gtrsim-0.8$ dex appear to have enhanced N/C abundance ratios, while more metal-poor clusters fall on a similar relation as Milky Way globular clusters (see dashed line in the middle panel of Fig. 13). This is evidence against an enrichment scenario dominated by hypernovae, which is expected to imprint higher $\mathrm{N} / \mathrm{C}$ ratios at lower metallicities. Instead, the opposite is observed and lends support to the notion that metalrich intermediate-mass AGB stars were given enough time ( $250 \mathrm{Myr})$ to eject and mix secondary nitrogen into the interstellar medium, before the $\mathrm{N}$-enhanced M 31 globular clusters formed.

In panel $\mathrm{c}$ of Fig. 13 we find evidence for a correlation of the $\mathrm{N} / \mathrm{C}$ ratio with $[\alpha / \mathrm{Fe}]$, in the sense that larger $\alpha$-enhancements are accompanied by higher N/C ratios. A dotted line shows a weighted linear least-square fit to the data. By itself this result does not favor either of the nitrogenenrichment mechanisms (hypernovae or intermediate-mass

\footnotetext{
${ }^{22}$ For completeness, we note that the Galactic globular cluster NGC 7078 shows a relatively high N/C ratio ( 4). This is in contrast to the results of Rauch et al. (2002) who measure a carbonenhancement and a nitrogen depletion (implying a small N/C ratio) in a sdO cluster star. Further high-resolution measurements for more stars in NGC 7078 are necessary to resolve this discrepancy.
} 
AGB stars). However, a significant hypernova enrichment is expected to produce an extreme $[\alpha / \mathrm{Fe}]$ signature with values $\gtrsim 1$ dex (e.g. Woosley \& Weaver 1995; Fryer et al. 2001), which is observed in some very metal-poor Galactic halo stars (e.g. Aoki et al. 2002), but not in the integrated light of nitrogenenhanced M 31 globular clusters.

\subsection{Chemical coincidence of globular cluster sub-populations}

It is intriguing that young M 31 and LMC globular clusters and some counterparts in the M 81/Sculptor-group sample show very similar $\mathrm{CN}$ indices at a given metallicity. Two scenarios seem plausible to explain the chemical similarity: 1) the globular cluster populations experienced similar enrichment histories, although they formed in galaxies of very different mass and morphology; or 2) the young M 31 clusters were stripped from accreted dwarf satellites.

Most of the young M 31 globular clusters reside in the outskirts of the M 31 disk and show radial velocities consistent with a kinematically cold thin-disk cluster population. However, it is relatively unlikely that an accretion event was so well aligned to leave no kinematical trace of a heated globular cluster sub-population. This makes the accretion scenario less likely.

Forbes et al. (2004) compile data on ages and metallicities of about a dozen Galactic globular clusters $(\sim 10 \%$ of the entire system), which are kinematically associated with giant stellar streams and dwarf galaxies in the process of accretion ${ }^{23}$. They find a range of ages and metallicities for accreted Galactic globular clusters, from $\sim 6$ to $13 \mathrm{Gyr}$, and metallicities from $[\mathrm{Fe} / \mathrm{H}] \approx-2$ to solar values, which does not allow us to distinguish captured from in-situ formed globular clusters using only age and metallicity. However, these authors argue that the age-metallicity relation for dwarf galaxies (stars and clusters) are different from the relation of the remaining Milky Way globular clusters. Different age-metallicity relations point to different chemical enrichment histories. A comparison of the age-metallicity relations of the Milky Way and M 31 globular cluster systems will be the subject of a future paper, when more accurate data for the Galactic globular cluster system become available. At this point, we note that a distinct age-metallicity relation of accreted globular clusters does not exclude either of the two former scenarios (chemical coincidence and accretion).

\subsection{Formation scenarios}

There are several formation and assembly scenarios for globular cluster systems in spiral galaxies, which can be ordered in three different categories. Pre-galactic scenarios envision globular cluster formation in dense metal-poor clouds before the aggregation of their host galaxy (Peebles \& Dicke 1968).

\footnotetext{
${ }^{23}$ Note that the potentially accreted satellite, which might be associated with some of the young M 31 globular clusters, does not necessarily have to leave a luminous stellar component, and hence a traceable stellar stream.
}

In proto-galactic scenarios, globular clusters form in loose gas clumps during the coalescence of the host galaxy in more or less chaotic merging events (Searle \& Zinn 1978). The third scenario pictures an a posteriori assembly where a fraction of the cluster system is added to the in-situ formed globular cluster system through accretion of external globular clusters from satellite galaxies (Côté et al. 2000). It is clear that all models overlap in many aspects and all present valid formation mechanisms for at least some globular cluster sub-populations (i.e. halo, disk, bulge) in M 31.

The current data does not allow a clear-cut answer on the importance of each mechanism in the specific case of M 31, since our data does not sample a representative fraction of halo globular clusters. However, our study has revealed a globularcluster age distribution which is entirely inconsistent with the predictions of the pre-galactic and somewhat inconsistent with the proto-galactic scenario. The formation of intermediate-age globular clusters appears only plausible in an extended protogalactic scenario where the collapse of the gas clumps occurs over an extended period of time, i.e. for several Gyr: a scenario closely resembling the hierarchical assembly of galaxies. Hence, the pre- and proto-galactic models cannot solely describe the assembly of the M 31 globular cluster system. Because of their spatial concentration towards the inner $\sim 5 \mathrm{kpc}$, an accretion scenario also appears less likely as a formation mechanism for the intermediate-age sub-population.

It should be stressed that the formation of individual globular clusters might be at stark variance with the suggested formation pictures. For instance, one might picture the formation of intermediate-age globular clusters from material which was enriched in the disk and subsequently funneled into the core regions through bar instabilities. Upscaled versions of this scenario are observed in other spiral galaxies (e.g. Jogee et al. 2002). The fact that most of the intermediate-age globular clusters show radial velocities that are consistent with thin-disk kinematics lends support to this formation picture. Alternatively, one could imagine a two-phase collapse, similar to the one suggested by Forbes et al. (1997) for globular cluster systems in early-type galaxies. In this scenario, halo globular clusters form first from primordial gas during the proto-galactic phase. The first generation of stars enriches the remaining gas which later settles in a disk and forms the second generation of younger and more metal-rich globular clusters.

A fraction of young M 31 globular clusters can be explained by the accretion scenario, as we find a few objects in close projected vicinity of satellite galaxies and giant stellar streams (see Fig. 1). The majority of these young relatively metal-rich globular clusters, however, resides in the outskirts of M 31's disk and forms a kinematically cold sub-population. As discussed earlier it is rather unlikely, but not impossible, that a wellaligned, pro-grade merging event stripped these clusters from a dwarf satellite galaxy. It is also possible that we see evidence for a massive star-forming ring (see GALEX press-release images $^{24}$ ) in which young star-clusters are formed from recently compressed molecular clouds, which might be triggered by recent tidal stress.

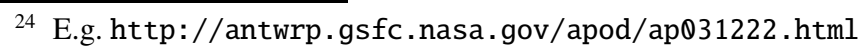


For completeness we note that the old globular cluster subpopulation has a wide range of metallicities and its spatial distribution is consistent with a halo population.

Chemical compositions of intermediate-age and young M 31 globular clusters are consistent with a formation on extended timescales. The chemical signatures of their old counterparts are expected to be the result of short star-formation events in which the interstellar medium was significantly polluted by the thermonuclear ashes of type-II supernovae. In particular, Li \& Burstein (2003) put forward the idea that the old M 31 globular-cluster population experienced significant enrichment by nitrogen-rich hypernova ejecta, motivated by the assumption that the nitrogen overabundance in metal-poor Galactic halo stars and old M 31 globular clusters has the same origin. Although conceptually plausible, we do not subscribe to this idea in its details. It is likely that hypernovae contributed significantly to the enrichment of the interstellar medium from which the most metal-poor Galactic stars $([\mathrm{Z} / \mathrm{H}] \lesssim-4)$ have formed. However, their metallicity is about two orders of magnitude lower than that of the most metal-poor known Galactic and M 31 globular cluster ${ }^{25}$. It is hard to believe that no moderate-mass $\left(\sim 10 M_{\odot}\right)$ type-II supernovae contributed to the chemical signatures of the most metal-poor globular clusters since the early epochs of chemical enrichment.

Furthermore, the hypernova scenario advocated by Li \& Burstein (2003), has difficulties in explaining the formation of the old metal-poor, but not nitrogen-enhanced M 31 globular clusters (such as B311, see Fig. 13; this globular cluster has the smallest $\mathrm{N} / \mathrm{C}$ ratio in our sample). It is conceivable that this might be a result of chemical variance in the very early epochs of chemical enrichment. Clearly, the hypernova scenario requires some detailed modeling and comparison with observations.

It would also be interesting to check if there is a range in nitrogen enhancement for Milky Way and other globular cluster systems at low metallicities, resembling the chemical variance during the very early star-formation epochs. We have embarked on a photometric and spectroscopic multi-wavelength study of globular clusters in the Milky Way and Local Group galaxies with ground and space-based telescopes and will address these issues in the future in more detail.

\section{Summary and conclusions}

The presence of a populous M 31 globular-cluster subsystem significantly younger than found in the Galactic globular cluster system is certainly the most surprising result of this study. Evidence for the counterpart globular cluster population in the Milky Way is missing, but we have found evidence for the presence of similar globular clusters in other spiral galaxies. Previous studies have shown that such intermediate-age and young globular clusters also exist in giant early-type (Puzia et al. 2002b; Puzia 2003a,b; Larsen et al. 2003; Bridges et al. 2003; Puzia et al. 2004b) and dwarf galaxies (e.g. Beasley et al. 2002).

\footnotetext{
25 The most metal-poor Galactic globular cluster NGC 5053 has a $[\mathrm{Fe} / \mathrm{H}] \approx-2.3 \mathrm{dex}$ (Harris 1996).
}

In general, the M 31 globular cluster system hosts a wider variety of ages and chemical compositions than the Galactic globular cluster system, and is therefore more representative for extragalactic stellar populations, especially for the comparison with stellar populations in early-type galaxies. It seems that globular cluster system in spiral galaxies might have experienced conceptually similar, but quantitatively different, formation and/or assembly histories. Some extreme cases might even resemble globular-cluster formation/assembly histories of early-type galaxies.

Below we summarize the main results of this work.

- In addition to a dominant population of globular clusters with ages $>10 \mathrm{Gyr}$ and metallicities from $[\mathrm{Z} / \mathrm{H}] \approx-2.0$ to solar values, we find a population $(\sim 20 \pm 7 \%$ of the observed sample) of intermediate-age globular clusters with ages between $\sim 5$ and 9 Gyr and a mean metallicity around -0.6 dex. A few of these objects have metallicities around -1.6 dex.

- We confirm the presence of young M 31 globular clusters recently identified by Beasley et al. (2004), which have ages $\lesssim 1 \mathrm{Gyr}$ and relatively high metallicities around -0.4 dex; however, two metal-poor young objects have also been found.

- The M 31 globular cluster system has a clearly super-solar mean $\alpha / \mathrm{Fe}$ abundance ratio of $+0.14 \pm 0.04$ dex with a dispersion $\sigma=0.32$ dex. However, the distribution appears bimodal with peaks around +0.2 and -0.03 dex, which have different dispersions, 0.19 and $0.50 \mathrm{dex}$, respectively.

- Intermediate-age and young globular clusters show roughly solar abundance ratios.

- We find evidence for an age- $[\alpha / \mathrm{Fe}]$ relation in the sense that younger clusters have smaller mean $[\alpha / \mathrm{Fe}]$ ratios. This is driven by the metal-rich intermediate-age and young cluster population with metallicities higher than $[\mathrm{Z} / \mathrm{H}] \approx-1.0 \mathrm{dex}$.

- The indices of globular clusters older than $\sim 5$ Gyr are consistent with a factor of three or higher in nitrogenabundance enhancement compared to their younger counterparts.

- Using kinematical data for a thin-disk model (Morrison et al. 2004) we find that the halo population of globular clusters is old ( $29 \mathrm{Gyr}$ ), has a bimodal metallicity distribution and super solar $\alpha / \mathrm{Fe}$-enhancement.

- Thin/thick-disk globular clusters have a wide range of ages and are predominantly metal-rich with a slightly smaller mean $[\alpha / \mathrm{Fe}]$ ratio.

- With the structural parameters of M 31 globular clusters measured by Barmby et al. (2002), we confirm a previously found correlation between half-light/tidal radius and globular clusters metallicity, which is most likely due to the correlation of half-light/tidal radius and galactocentric distance.

- Comparing the integrated light of the M 31 and Milky Way bulge, we find that both host metal-rich, $\alpha$-element enhanced and old stellar populations.

- We find indications for an enhancement in carbon and nitrogen in the central region of M 31, while the integrated 
light of outer M 31 stellar populations is consistent with a nitrogen enhancement only.

Acknowledgements. T.H.P. gratefully acknowledges support in form of an ESA Research Fellowship. K.M.P. acknowledges support of the Natural Sciences and Research Council of Canada in form of an NSERC Fellowship. T.J.B. thanks David Hanes for financial assistance during his stay at Queen's University. We are indebted to Knut Olsen for re-measuring and calibrating Lick indices of globular clusters in Sculptor-group spiral galaxies and to Francesca De Angeli for providing CMD ages and metallicities prior to publication. We thank Nicole Homeier and Maurizio Paolillo for reading early versions of the draft. It is a pleasure to acknowledge fruitful discussions with Rupali Chandar, Paul Goudfrooij, and Tom Brown on the results of this work. We thank the anonymous referee for useful comments and suggestions that improved the paper. This research has made use of the SIMBAD database, operated at CDS, Strasbourg, France. The Digitized Sky Surveys were produced at the Space Telescope Science Institute under US Government grant NAG W-2166. The images of these surveys are based on photographic data obtained using the Oschin Schmidt Telescope on Palomar Mountain and the UK Schmidt Telescope. The plates were processed into the present compressed digital form with the permission of these institutions.

\section{References}

Alongi, M., Bertelli, G., Bressan, A., et al. 1993, A\&AS, 97, 851 Aoki, W., Norris, J. E., Ryan, S. G., Beers, T. C., \& Ando, H. 2002, ApJ, 576, L141

Ashman, K. A., Bird, C. M., \& Zepf, S. E. 1994, AJ, 108, 2348

Ashman, K. M., \& Zepf, S. E. 1998, Globular Cluster Systems (Cambridge University Press)

Baade, W. 1944, ApJ, 100, 137

Barbuy, B., Renzini, A., Ortolani, S., Bica, E., \& Guarnieri, M. D. 1999, A\&A, 341, 539

Barmby, P., \& Huchra, J. P. 2001, AJ, 122, 2458

Barmby, P., Huchra, J. P., Brodie, J. P., et al. 2000, AJ, 119, 727

Barmby, P., Huchra, J. P., \& Brodie, J. P. 2001, AJ, 121, 1482

Barmby, P., Holland, S., \& Huchra, J. P. 2002, AJ, 123, 1937

Beasley, M. A., Hoyle, F., \& Sharples, R. M. 2002, MNRAS, 336, 168

Beasley, M. A., Brodie, J. P., Strader, J., et al. 2004, AJ, 128, 1623

Bellazzini, M., Cacciari, C., Federici, L., Fusi Pecci, F., \& Rich, M. 2003, A\&A, 405, 867

Bridges, T. J. 1998, in Fiber Optics in Astronomy III, ed. S. Arribas, E. Mediavilla, \& F. Watson (San Francisco: ASP), ASP Conf. Ser., 152, 104

Bridges, T., Beasley, M., Faifer, F., et al. 2003, Extragalactic Globular Clusters and their Host Galaxies, 25th meeting of the IAU, Joint Discussion 6, 17 July 2003, Sydney, Australia, 6

Brodie, J. P., \& Huchra, J. P. 1990, ApJ, 362, 503

Brodie, J. P., \& Huchra, J. P. 1991, ApJ, 379, 157

Brown, T. M., Ferguson, H. C., Smith, E., et al. 2003, ApJ, 592, L17

Bruzual, G., \& Charlot, S. 2003, MNRAS, 344, 1000

Burstein, D. 1987, Nearly Normal Galaxies. From the Planck Time to the Present, 47

Burstein, D., Faber, S. M., Gaskell, C. M., \& Krumm, N. 1984, ApJ, 287, 586

Burstein, D., Li, Y., Freeman, K. C., et al. 2004, ApJ, 614, 158

Carretta, E., Cohen, J. G., Gratton, R. G., \& Behr, B. B. 2001, AJ, 122, 1469

Carretta, E., Gratton, R. G., Bragaglia, A., Bonifacio, P., \& Pasquini, L. 2004, A\&A, 416, 925
Chaboyer, B., Demarque, P., Kernan, P. J., \& Krauss, L. M. 1998, ApJ, 494, 96

Chernoff, D. F., \& Djorgovski, S. 1989, ApJ, 339, 904

Christlieb, N., Bessell, M. S., Beers, T. C., et al. 2002, Nature, 419, 904

Christlieb, N., Gustafsson, B., Korn, A. J., et al. 2004, ApJ, 603, 708

Cohen, J. G. 2004, AJ, 127, 1545

Cohen, J. G., Blakeslee, J. P., \& Ryzhov, A. 1998, ApJ, 496, 808

Cohen, J. G., Gratton, R. G., Behr, B. B., \& Carretta, E. 1999, ApJ, 523, 739

Côté, P. 1999, AJ, 118, 406

Côté, P., Marzke, R. O., West, M. J., \& Minniti, D. 2000, ApJ, 533, 869

Davidge, T. J. 1990a, ApJ, 351, L37

Davidge, T. J. 1990b, AJ, 99, 561

Davidge, T. J., de Robertis, M. M., \& Yee, H. K. C. 1990, AJ, 100, 1143

Djorgovski, S. 1995, ApJ, 438, L29

Durrell, P. R., Harris, W. E., Geisler, D., \& Pudritz, R. E. 1996, AJ, 112, 972

Durrell, P. R., Harris, W. E., \& Pritchet, C. J. 2001, AJ, 121, 2557

Durrell, P. R., Harris, W. E., \& Pritchet, C. J. 2004, AJ, 128, 260

Edvardsson, B., Andersen, J., Gustafsson, B., et al. 1993, A\&A, 275, 101

Esteban, C., \& Peimbert, M. 1995, A\&A, 300, 78

Ferguson, A. M. N., Irwin, M. J., Ibata, R. A., Lewis, G. F., \& Tanvir, N. R. 2002, AJ, 124, 1452

Ferraro, F. R., Fusi Pecci, F., Testa, V., et al. 1995, MNRAS, 272, 391

Font, A. S., Navarro, J. F., Stadel, J., \& Quinn, T. 2001, ApJ, 563, L1

Forbes, D. A., Brodie, J. P., \& Grillmair, C. J. 1997, AJ, 113, 1652

Forbes, D. A., Strader, J., \& Brodie, J. P. 2004, AJ, 127, 3394

Fryer, C. L., Woosley, S. E., \& Heger, A. 2001, ApJ, 550, 372

Fuhrmann, K. 1998, A\&A, 338, 161

Galleti, S., Federici, L., Bellazzini, M., Fusi Pecci, F., \& Macrina, S. 2004, A\&A, 416, 917

Greggio, L. 1997, MNRAS, 285, 151

Harris, W. E. 1996, AJ, 112, 1487

Harris, W. E. 2001, Star Clusters, 223

Heger, A., Woosley, S. E., \& Waters, R. 2000, The First Stars. Proceedings of the MPA/ESO Workshop held at Garching, Germany, 4-6 August 1999, ed. A. Weiss, T. G. Abel, \& V. Hill (Springer), 121

Heger, A., Fryer, C. L., Woosley, S. E., Langer, N., \& Hartmann, D. H. 2003, ApJ, 591, 288

Hempel, M., Hilker, M., Kissler-Patig, M., et al. 2003, A\&A, 405, 487

Holland, S., Fahlman, G. G., \& Richer, H. B. 1996, AJ, 112, 1035

Hopper, P. J. C. 1995, Ph.D. Thesis

Huchra, J. P., Brodie, J. P., \& Kent, S. M. 1991, ApJ, 370, 495

Ibata, R., Irwin, M., Lewis, G., Ferguson, A. M. N., \& Tanvir, N. 2001, Nature, 412, 49

Ibata, R., Chapman, S., Ferguson, A. M. N., et al. 2004, MNRAS, 351, 117

Jiang, L., Ma, J., Zhou, X., et al. 2003, AJ, 125, 727

Jogee, S., Shlosman, I., Laine, S., et al. 2002, ApJ, 575, 156

Jordán, A. 2004, ApJ, 613, L117

King, I. 1962, AJ, 67, 471

Kundu, A., \& Whitmore, B. C. 1998, AJ, 116, 2841

Kuntschner, H. 1998, Ph.D. Thesis

Kuntschner, H. 2000, MNRAS, 315, 184

Kuntschner, H., Ziegler, B. L., Sharples, R. M., Worthey, G., \& Fricke, K. J. 2002, A\&A, 395, 761

Larsen, S. S., \& Brodie, J. P. 2003, ApJ, 593, 340 
Larsen, S. S., Brodie, J. P., Huchra, J. P., Forbes, D. A., \& Grillmair, C. J. 2001, AJ, 121, 2974

Larsen, S. S., Brodie, J. P., Beasley, M. A., et al. 2003, ApJ, 585, 767

Li, Y., \& Burstein, D. 2003, ApJ, 598, L103

Li, Y., Mac Low, M., \& Klessen, R. S. 2004, ApJ, 614, L29

Mackey, A. D., \& Gilmore, G. F. 2003, MNRAS, 338, 85

Maeder, A. 1992, A\&A, 264, 105

Maraston, C., Greggio, L., Renzini, A., et al. 2003, A\&A, 400, 823

Matteucci, F. 1994, A\&A, 288, 57

Matteucci, F., \& Recchi, S. 2001, ApJ, 558, 351

McConnachie, A. W., Irwin, M. J., Ibata, R. A., et al. 2003, MNRAS, 343, 1335

McConnachie, A. W., Irwin, M. J., Lewis, G. F., et al. 2004, MNRAS, 351, L94

Morgan, W. W., \& Mayall, N. U. 1957, PASP, 69, 291

Morrison, H. L., Harding, P., Perrett, K., \& Hurley-Keller, D. 2004, ApJ, 603, 87

Norris, J., \& Pilachowski, C. A. 1985, ApJ, 299, 295

Norris, J. E., Ryan, S. G., \& Beers, T. C. 2001, Apj, 561, 1034

Norris, J. E., Ryan, S. G., Beers, T. C., Aoki, W., \& Ando, H. 2002, ApJ, 569, L107

O'Connell, R. W. 1973, AJ, 78, 1074

Olsen, K. A. G., Miller, B. W., Suntzeff, N. B., Schommer, R. A., \& Bright, J. 2004, AJ, 127, 2674

Origlia, L., \& Rich, R. M. 2004, AJ, 127, 3422

Origlia, L., Rich, R. M., \& Castro, S. 2002, AJ, 123, 1559

Peebles, P. J. E., \& Dicke, R. H. 1968, ApJ, 154, 891

Perrett, K. M., Bridges, T. J., Hanes, D. A., et al. 2002, AJ, 123, 2490

Perrett, K. M., Stiff, D. A., Hanes, D. A., \& Bridges, T. J. 2003, ApJ, 589, 790

Pettini, M., Ellison, S. L., Bergeron, J., \& Petitjean, P. 2002, A\&A, 391, 21

Ponder, J. M., Burstein, D., O’Connell, R. W., et al. 1998, AJ, 116, 2297

Puzia, T. H. 2003a, Extragalactic Globular Cluster Systems, Proceedings of the ESO Workshop held in Garching, Germany, ed. M. Kissler-Patig, 267

Puzia, T. H. 2003b, Ph.D. Thesis, Ludwig-Maximilians-Universität, München

Puzia, T. H., Kissler-Patig, M., Brodie, J. P., \& Huchra, J. P. 1999, AJ, 118, 2734

Puzia, T. H., Zepf, S. E., Kissler-Patig, M., et al. 2002a, A\&A, 391, 453

Puzia, T. H., Saglia, R. P., Kissler-Patig, M., et al. 2002b, A\&A, 395, 45

Puzia, T. H., Kissler-Patig, M., Thomas, D., et al. 2004a, A\&A, 415, 123
Puzia, T. H., et al. 2004b, A\&A, submitted

Rabin, D. M. 1981, Ph.D. Thesis

Rauch, T., Heber, U., \& Werner, K. 2002, A\&A, 381, 1007

Renzini, A., \& Buzzoni, A. 1986, ASSL Vol. 122: Spectral Evolution of Galaxies, 195

Rich, R. M., Reitzel, D. B., Guhathakurta, P., Gebhardt, K., \& Ho, L. C. 2004, AJ, 127, 2139

Salaris, M., \& Weiss, A. 2002, A\&A, 388, 492

Sánchez-Blázquez, P., Gorgas, J., Cardiel, N., Cenarro, J., \& González, J. J. 2003, ApJ, 590, L91

Sarajedini, A., \& Van Duyne, J. 2001, AJ, 122, 2444

Schroder, L. L., Brodie, J. P., Kissler-Patig, M., Huchra, J. P., \& Phillips, A. C. 2002 , AJ, 123, 2473

Schwarzkopf, U., \& Dettmar, R.-J. 2000, A\&A, 361, 451

Schwarzkopf, U., \& Dettmar, R.-J. 2001, A\&A, 373, 402

Searle, L., \& Zinn, R. 1978, Apj, 225, 357

Silverman, B. W. 1986, Density Estimation for Statistics and Data Analysis, Chap and Hall/CRC Press, Inc.

Spinrad, H., \& Schweizer, F. 1972, ApJ, 171, 403

Thomas, D., Maraston, C., \& Bender, R. 2003, MNRAS, 339, 897

Thomas, D., Maraston, C., \& Korn, A. 2004, MNRAS, 351, L19

Tomkin, J., \& Lambert, D. L. 1984, ApJ, 279, 220

Trager, S. C., Worthey, G., Faber, S. M., Burstein, D., \& Gonzalez, J. J. 1998, ApJS, 116, 1

Trager, S. C., Faber, S. M., Worthey, G., \& González, J. J. 2000, AJ, 119,1645

Tripicco, M. J. 1989, AJ, 97, 735

Tripicco, M. J., \& Bell, R. A. 1995, AJ, 110, 3035

Tsujimoto, T. 2004, ApJ, 611, L17

van den Bergh, S. 1969, ApJS, 19, 145

van den Bergh, S. 2000, ApJ, 530, 777

van den Bergh, S., Morbey, C., Pazder, J. 1991, ApJ, 375, 594

van den Hoek, L. B., \& Groenewegen, M. A. T. 1997, A\&AS, 123, 305

Vazdekis, A., \& Arimoto, N. 1999, ApJ, 525, 144

Velazquez, H., \& White, S. D. M. 1999, MNRAS, 304, 254

Widrow, L. M., Perrett, K. M., \& Suyu, S. H. 2003, ApJ, 588, 311

Woosley, S. E., \& Weaver, T. A. 1982, NATO ASIC Proc. 90: Supernovae: A Survey of Current Research, 79

Woosley, S. E., \& Weaver, T. A. 1995, ApJS, 101, 181

Worthey, G., Faber, S. M., \& Gonzalez, J. J. 1992, ApJ, 398, 69

Worthey, G., Faber, S. M., Gonzalez, J. J., \& Burstein, D. 1994, ApJS, 94,687

Worthey, G., \& Ottaviani, D. L. 1997, ApJS, 111, 377

Zibetti, S., White, S. D. M., \& Brinkmann, J. 2004, MNRAS, 347, 556 
T. H. Puzia et al.: New light on the formation and evolution of M 31 and its globular cluster system, Online Material p 1

\section{Online Material}


T. H. Puzia et al.: New light on the formation and evolution of M 31 and its globular cluster system, Online Material p 2

\section{Appendix A: Lick index measurements, ages, metallicities, and $[\alpha / \mathrm{Fe}]$ ratios}

Table A.1. Lick indices for our sample M 31 globular clusters. Indices were computed with the passband definitions of Worthey et al. (1994) and for the higher-order Balmer lines the definitions of Worthey \& Ottaviani (1997). We adopted the nomenclature of Galleti et al. (2004) for all our objects. Note that B166 is suspected by some studies to be a foreground star. Since the case is not clear, we mark this object as potentially misclassified but keep it in our globular cluster sample. However, we point out that all other objects were classified by several independent studies as genuine globular clusters, based on their colors and radial velocities (see Galleti et al. 2004).

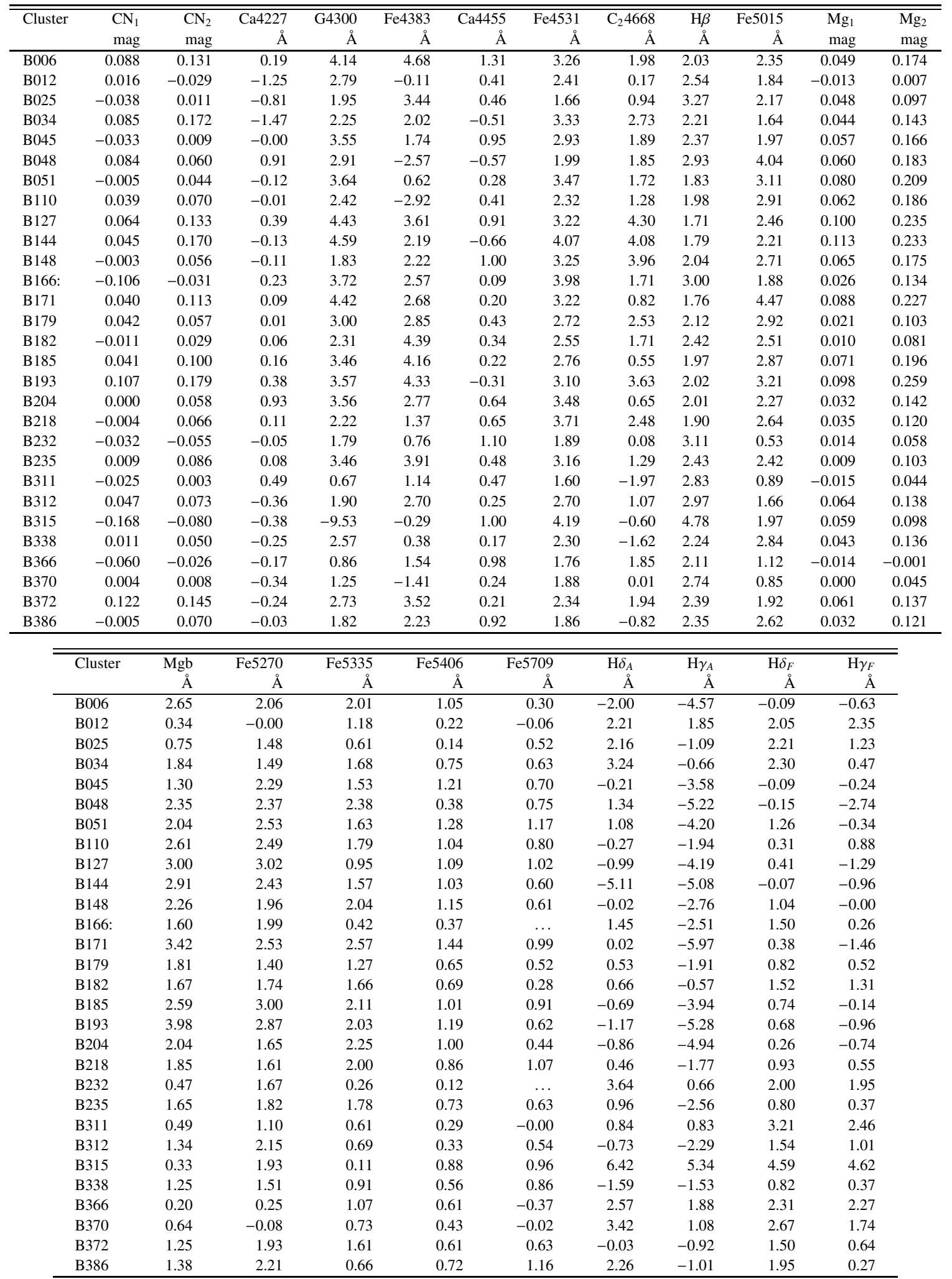


T. H. Puzia et al.: New light on the formation and evolution of M 31 and its globular cluster system, Online Material p 3

Table A.2. Statistical errors of Lick indices for our sample globular clusters.

\begin{tabular}{|c|c|c|c|c|c|c|c|c|c|c|c|c|}
\hline Cluster & $\begin{array}{l}\mathrm{CN}_{1} \\
\mathrm{mag}\end{array}$ & $\begin{array}{l}\mathrm{CN}_{2} \\
\mathrm{mag}\end{array}$ & $\begin{array}{r}\text { Ca4227 } \\
\AA\end{array}$ & $\begin{array}{r}\mathrm{G} 4300 \\
\AA\end{array}$ & $\begin{array}{r}\text { Fe4383 } \\
\AA\end{array}$ & $\begin{array}{r}\text { Ca4455 } \\
\AA\end{array}$ & $\begin{array}{r}\text { Fe4531 } \\
\AA\end{array}$ & $\begin{array}{r}\mathrm{C}_{2} 4668 \\
\AA\end{array}$ & $\begin{array}{r}\mathrm{H} \beta \\
\AA\end{array}$ & $\begin{array}{r}\text { Fe5015 } \\
\AA\end{array}$ & $\begin{array}{l}\mathrm{Mg}_{1} \\
\mathrm{mag}\end{array}$ & $\begin{array}{l}\mathrm{Mg}_{2} \\
\mathrm{mag}\end{array}$ \\
\hline B006 & 0.013 & 0.019 & 0.58 & 0.64 & 0.31 & 0.35 & 0.45 & 0.54 & 0.56 & 0.59 & 0.016 & 0.016 \\
\hline B012 & 0.012 & 0.018 & 0.65 & 0.87 & 0.25 & 0.28 & 0.33 & 0.50 & 0.51 & 0.53 & 0.011 & 0.011 \\
\hline B025 & 0.020 & 0.040 & 1.41 & 1.51 & 0.53 & 0.56 & 0.62 & 0.77 & 0.78 & 0.84 & 0.019 & 0.019 \\
\hline B034 & 0.019 & 0.027 & 0.85 & 0.90 & 0.26 & 0.29 & 0.32 & 0.46 & 0.47 & 0.47 & 0.011 & 0.011 \\
\hline B045 & 0.005 & 0.007 & 0.25 & 0.35 & 0.33 & 0.37 & 0.41 & 0.56 & 0.56 & 0.60 & 0.014 & 0.014 \\
\hline B048 & 0.014 & 0.022 & 0.69 & 0.85 & 0.56 & 0.60 & 0.67 & 0.76 & 0.77 & 0.84 & 0.019 & 0.019 \\
\hline B051 & 0.015 & 0.017 & 0.56 & 0.72 & 0.41 & 0.45 & 0.50 & 0.66 & 0.67 & 0.69 & 0.016 & 0.016 \\
\hline B110 & 0.010 & 0.014 & 0.49 & 0.53 & 0.44 & 0.45 & 0.47 & 0.50 & 0.51 & 0.52 & 0.013 & 0.013 \\
\hline B127 & 0.002 & 0.004 & 0.12 & 0.13 & 0.16 & 0.16 & 0.17 & 0.20 & 0.20 & 0.21 & 0.004 & 0.004 \\
\hline B144 & 0.004 & 0.009 & 0.29 & 0.41 & 0.26 & 0.30 & 0.32 & 0.45 & 0.46 & 0.46 & 0.010 & 0.011 \\
\hline B148 & 0.010 & 0.011 & 0.36 & 0.39 & 0.27 & 0.30 & 0.35 & 0.37 & 0.37 & 0.38 & 0.010 & 0.010 \\
\hline B166: & 0.007 & 0.011 & 0.38 & 0.43 & 0.35 & 0.41 & 0.49 & 0.66 & 0.67 & 0.69 & 0.016 & 0.016 \\
\hline B171 & 0.007 & 0.011 & 0.33 & 0.39 & 0.44 & 0.45 & 0.49 & 0.61 & 0.62 & 0.63 & 0.014 & 0.014 \\
\hline B179 & 0.005 & 0.007 & 0.22 & 0.25 & 0.23 & 0.25 & 0.30 & 0.33 & 0.34 & 0.36 & 0.009 & 0.010 \\
\hline B182 & 0.005 & 0.008 & 0.26 & 0.29 & 0.21 & 0.25 & 0.32 & 0.41 & 0.42 & 0.44 & 0.011 & 0.011 \\
\hline B185 & 0.004 & 0.008 & 0.25 & 0.27 & 0.44 & 0.57 & 0.61 & 0.75 & 0.75 & 0.76 & 0.016 & 0.016 \\
\hline B193 & 0.005 & 0.015 & 0.46 & 0.52 & 0.27 & 0.31 & 0.35 & 0.53 & 0.53 & 0.54 & 0.013 & 0.013 \\
\hline B204 & 0.004 & 0.007 & 0.24 & 0.28 & 0.20 & 0.23 & 0.26 & 0.31 & 0.32 & 0.34 & 0.009 & 0.009 \\
\hline B218 & 0.004 & 0.006 & 0.19 & 0.23 & 0.17 & 0.19 & 0.21 & 0.29 & 0.29 & 0.30 & 0.007 & 0.007 \\
\hline B232 & 0.010 & 0.016 & 0.53 & 0.60 & 0.41 & 0.43 & 0.54 & 0.66 & 0.66 & 0.69 & 0.015 & 0.015 \\
\hline B235 & 0.013 & 0.017 & 0.56 & 0.80 & 0.37 & 0.46 & 0.54 & 0.61 & 0.62 & 0.65 & 0.018 & 0.018 \\
\hline B311 & 0.005 & 0.011 & 0.38 & 0.40 & 0.42 & 0.43 & 0.44 & 0.52 & 0.52 & 0.53 & 0.012 & 0.012 \\
\hline B312 & 0.010 & 0.011 & 0.36 & 0.47 & 0.38 & 0.39 & 0.41 & 0.43 & 0.43 & 0.45 & 0.010 & 0.010 \\
\hline B315 & 0.010 & 0.027 & 0.97 & 0.99 & 0.27 & 0.30 & 0.35 & 0.40 & 0.40 & 0.46 & 0.011 & 0.011 \\
\hline B338 & 0.006 & 0.012 & 0.38 & 0.52 & 0.58 & 0.70 & 0.72 & 0.75 & 0.75 & 0.79 & 0.018 & 0.018 \\
\hline B366 & 0.024 & 0.025 & 0.84 & 0.94 & 0.28 & 0.33 & 0.44 & 0.53 & 0.55 & 0.58 & 0.015 & 0.015 \\
\hline B370 & 0.006 & 0.016 & 0.54 & 0.61 & 0.57 & 0.58 & 0.66 & 0.71 & 0.71 & 0.72 & 0.015 & 0.015 \\
\hline B372 & 0.013 & 0.023 & 0.73 & 0.77 & 0.30 & 0.36 & 0.48 & 0.63 & 0.63 & 0.65 & 0.016 & 0.016 \\
\hline B386 & 0.003 & 0.012 & 0.41 & 0.46 & 0.32 & 0.35 & 0.39 & 0.51 & 0.51 & 0.52 & 0.012 & 0.012 \\
\hline
\end{tabular}

\begin{tabular}{|c|c|c|c|c|c|c|c|c|c|}
\hline Cluster & $\begin{array}{c}\mathrm{Mgb} \\
\AA\end{array}$ & $\begin{array}{r}\text { Fe5270 } \\
\AA\end{array}$ & $\begin{array}{r}\text { Fe5335 } \\
\AA\end{array}$ & $\begin{array}{r}\text { Fe5406 } \\
\AA\end{array}$ & $\begin{array}{r}\text { Fe5709 } \\
\AA\end{array}$ & $\begin{array}{r}\mathrm{H} \delta_{A} \\
\AA\end{array}$ & $\begin{array}{r}\mathrm{H} \gamma_{A} \\
\AA\end{array}$ & $\begin{array}{r}\mathrm{H} \delta_{F} \\
\AA\end{array}$ & $\begin{array}{r}\mathrm{H} \gamma_{F} \\
\AA\end{array}$ \\
\hline B006 & 0.62 & 0.63 & 0.63 & 0.63 & 0.64 & 0.98 & 1.00 & 1.09 & 1.10 \\
\hline B012 & 0.53 & 0.54 & 0.55 & 0.56 & 0.56 & 1.22 & 1.43 & 1.56 & 1.59 \\
\hline B025 & 0.87 & 0.88 & 0.88 & 0.88 & 0.89 & 1.73 & 1.75 & 1.79 & 1.80 \\
\hline B034 & 0.49 & 0.50 & 0.50 & 0.50 & 0.50 & 1.13 & 1.22 & 1.28 & 1.29 \\
\hline B045 & 0.64 & 0.64 & 0.64 & 0.65 & 0.65 & 0.60 & 0.69 & 0.76 & 0.77 \\
\hline B048 & 0.86 & 0.86 & 0.88 & 0.88 & 0.89 & 1.10 & 1.20 & 1.24 & 1.27 \\
\hline B051 & 0.73 & 0.74 & 0.74 & 0.74 & 0.74 & 1.05 & 1.07 & 1.09 & 1.09 \\
\hline B110 & 0.52 & 0.53 & 0.53 & 0.53 & 0.53 & 0.85 & 1.22 & 1.31 & 1.34 \\
\hline B127 & 0.21 & 0.21 & 0.21 & 0.21 & 0.21 & 0.23 & 0.30 & 0.30 & 0.31 \\
\hline B144 & 0.47 & 0.47 & 0.48 & 0.48 & 0.48 & 0.81 & 0.82 & 0.83 & 0.86 \\
\hline B148 & 0.39 & 0.39 & 0.39 & 0.39 & 0.39 & 0.77 & 0.78 & 0.78 & 0.79 \\
\hline B166: & 0.70 & 0.71 & 0.72 & 0.72 & & 0.59 & 0.61 & 0.63 & 0.63 \\
\hline B171 & 0.65 & 0.65 & 0.65 & 0.66 & 0.66 & 0.53 & 0.57 & 0.67 & 0.69 \\
\hline B179 & 0.37 & 0.37 & 0.37 & 0.37 & 0.38 & 0.30 & 0.32 & 0.33 & 0.35 \\
\hline B182 & 0.46 & 0.47 & 0.47 & 0.47 & 0.47 & 0.46 & 0.56 & 0.57 & 0.58 \\
\hline B185 & 0.76 & 0.76 & 0.76 & 0.76 & 0.76 & 0.66 & 0.76 & 0.76 & 0.77 \\
\hline B193 & 0.57 & 0.57 & 0.57 & 0.58 & 0.58 & 0.88 & 0.93 & 0.94 & 0.95 \\
\hline B204 & 0.35 & 0.35 & 0.36 & 0.36 & 0.36 & 0.42 & 0.44 & 0.46 & 0.47 \\
\hline B218 & 0.30 & 0.30 & 0.31 & 0.31 & 0.31 & 0.28 & 0.30 & 0.35 & 0.37 \\
\hline B232 & 0.70 & 0.70 & 0.70 & 0.71 & $\ldots$ & 0.76 & 0.77 & 0.84 & 0.84 \\
\hline B235 & 0.66 & 0.68 & 0.70 & 0.70 & 0.70 & 1.16 & 0.73 & 1.35 & 0.74 \\
\hline B311 & 0.56 & 0.56 & 0.56 & 0.56 & 0.57 & 0.75 & 0.76 & 0.79 & 0.80 \\
\hline B312 & 0.45 & 0.45 & 0.45 & 0.45 & 0.45 & 0.83 & 0.88 & 0.89 & 0.90 \\
\hline B315 & 0.47 & 0.48 & 0.50 & 0.51 & 0.51 & 1.03 & 1.05 & 1.06 & 1.06 \\
\hline B338 & 0.82 & 0.83 & 0.83 & 0.83 & 0.83 & 0.61 & 0.63 & 0.65 & 0.73 \\
\hline B366 & 0.59 & 0.59 & 0.60 & 0.60 & 0.60 & 1.17 & 1.19 & 1.21 & 1.22 \\
\hline B370 & 0.73 & 0.73 & 0.74 & 0.74 & 0.74 & 0.94 & 1.03 & 1.04 & 1.06 \\
\hline B372 & 0.66 & 0.67 & 0.67 & 0.67 & 0.67 & 0.91 & 0.93 & 0.94 & 0.95 \\
\hline B386 & 0.54 & 0.54 & 0.54 & 0.54 & 0.54 & 0.51 & 0.63 & 0.64 & 0.66 \\
\hline
\end{tabular}


T. H. Puzia et al.: New light on the formation and evolution of M 31 and its globular cluster system, Online Material p 4

Table A.3. Ages, Metallicities, and $[\alpha / \mathrm{Fe}]$ ratios for M 31 globular clusters. We point out that the quoted uncertainties are the propagated statistical uncertainties of the data in combination with the SSP models used in this work. Systematic uncertainties of the presented parameters are of the order (for $[\mathrm{Z} / \mathrm{H}]$ and $[\alpha / \mathrm{Fe}]$ ) or larger (for age) than the statistical errors and are discussed in Sect. 3.3.

\begin{tabular}{|c|c|c|c|c|}
\hline Cluster & $\mathrm{D}^{a}$ & Age [Gyr] & {$[\mathrm{Z} / \mathrm{H}]$} & {$[\alpha / \mathrm{Fe}]$} \\
\hline B006 & 1 & $12.4 \pm 2.8$ & $-0.35 \pm 0.14$ & $0.18 \pm 0.18$ \\
\hline B012 & 1 & $10.2 \pm 2.9$ & $-2.00 \pm 0.30$ & $-0.96 \pm 1.20$ \\
\hline B025 & 1 & $9.6 \pm 3.3$ & $-1.17 \pm 0.38$ & $0.43 \pm 0.34$ \\
\hline B034 & 1 & $8.1 \pm 3.9$ & $-0.62 \pm 0.15$ & $0.31 \pm 0.17$ \\
\hline B045 & 1 & $10.6 \pm 2.7$ & $-0.50 \pm 0.11$ & $0.22 \pm 0.19$ \\
\hline B048 & 1 & $7.5 \pm 3.6$ & $-0.20 \pm 0.30$ & $0.04 \pm 0.22$ \\
\hline B051 & 1 & $11.3 \pm 3.2$ & $-0.40 \pm 0.17$ & $0.32 \pm 0.20$ \\
\hline B110 & 1 & $6.2 \pm 2.7$ & $-0.23 \pm 0.18$ & $0.19 \pm 0.13$ \\
\hline B127 & 1 & $13.3 \pm 1.7$ & $-0.22 \pm 0.05$ & $0.45 \pm 0.05$ \\
\hline B144 & 1 & $14.4 \pm 3.0$ & $-0.25 \pm 0.10$ & $0.45 \pm 0.12$ \\
\hline B148 & 1 & $11.1 \pm 3.1$ & $-0.47 \pm 0.12$ & $0.22 \pm 0.11$ \\
\hline B166: & 1 & $9.3 \pm 2.9$ & $-0.52 \pm 0.13$ & $0.56 \pm 0.27$ \\
\hline B171 & 1 & $10.8 \pm 2.7$ & $-0.05 \pm 0.21$ & $0.12 \pm 0.15$ \\
\hline B179 & 1 & $11.2 \pm 1.6$ & $-0.67 \pm 0.07$ & $0.16 \pm 0.18$ \\
\hline B182 & 1 & $7.1 \pm 4.3$ & $-0.61 \pm 0.19$ & $-0.48 \pm 0.23$ \\
\hline B185 & 1 & $5.8 \pm 4.0$ & $-0.12 \pm 0.28$ & $0.01 \pm 0.18$ \\
\hline B193 & 1 & $8.3 \pm 3.6$ & $0.08 \pm 0.21$ & $0.29 \pm 0.12$ \\
\hline B204 & 1 & $12.3 \pm 1.8$ & $-0.44 \pm 0.05$ & $0.00 \pm 0.12$ \\
\hline B218 & 1 & $11.9 \pm 2.6$ & $-0.65 \pm 0.12$ & $-0.08 \pm 0.12$ \\
\hline B232 & 1 & $9.0 \pm 3.3$ & $-1.52 \pm 0.52$ & $-0.09 \pm 0.66$ \\
\hline B235 & 1 & $10.2 \pm 3.8$ & $-0.63 \pm 0.23$ & $-0.27 \pm 0.31$ \\
\hline B311 & 1 & $11.2 \pm 2.7$ & $-1.53 \pm 0.38$ & $-0.51 \pm 0.85$ \\
\hline B312 & 1 & $9.4 \pm 1.7$ & $-0.58 \pm 0.09$ & $0.40 \pm 0.17$ \\
\hline B315 & 1 & $3.5 \pm 2.2$ & $-1.54 \pm 0.61$ & $0.46 \pm 0.23$ \\
\hline B338 & 1 & $12.2 \pm 2.6$ & $-0.65 \pm 0.19$ & $0.55 \pm 0.29$ \\
\hline B366 & 1 & $11.0 \pm 3.0$ & $-1.84 \pm 0.32$ & $-0.91 \pm 1.18$ \\
\hline B370 & 1 & $9.1 \pm 2.5$ & $-1.73 \pm 0.21$ & $0.99 \pm 0.95$ \\
\hline B372 & 1 & $11.9 \pm 3.5$ & $-0.77 \pm 0.26$ & $0.09 \pm 0.24$ \\
\hline B386 & 1 & $10.9 \pm 3.2$ & $-0.79 \pm 0.22$ & $0.25 \pm 0.22$ \\
\hline B126 & 2 & $7.2 \pm 3.1$ & $-1.25 \pm 0.21$ & $0.15 \pm 0.19$ \\
\hline B134 & 2 & $11.3 \pm 1.8$ & $-0.54 \pm 0.06$ & $0.09 \pm 0.10$ \\
\hline B158 & 2 & $11.3 \pm 1.5$ & $-0.51 \pm 0.05$ & $0.20 \pm 0.07$ \\
\hline B163 & 2 & $7.7 \pm 1.0$ & $0.09 \pm 0.05$ & $0.20 \pm 0.03$ \\
\hline B222 & 2 & $1.1 \pm 0.4$ & $-0.46 \pm 0.13$ & $0.20 \pm 0.13$ \\
\hline B225 & 2 & $9.9 \pm 1.2$ & $-0.21 \pm 0.04$ & $0.26 \pm 0.03$ \\
\hline B234 & 2 & $11.4 \pm 2.0$ & $-0.47 \pm 0.05$ & $0.07 \pm 0.09$ \\
\hline B292 & 2 & $9.2 \pm 3.3$ & $-1.53 \pm 0.39$ & $0.11 \pm 0.31$ \\
\hline B301 & 2 & $4.1 \pm 3.7$ & $-0.61 \pm 0.13$ & $-0.12 \pm 0.24$ \\
\hline B304 & 2 & $13.2 \pm 3.1$ & $-1.27 \pm 0.37$ & $-0.12 \pm 0.29$ \\
\hline B310 & 2 & $10.8 \pm 3.1$ & $-1.55 \pm 0.37$ & $-0.33 \pm 0.39$ \\
\hline B313 & 2 & $11.2 \pm 1.2$ & $-0.53 \pm 0.03$ & $0.28 \pm 0.11$ \\
\hline B314 & 2 & $1.0 \pm 0.1$ & $-0.28 \pm 0.06$ & $0.07 \pm 0.23$ \\
\hline B321 & 2 & $1.0 \pm 0.1$ & $-1.79 \pm 0.08$ & $0.20 \pm 0.31$ \\
\hline B322 & 2 & $2.3 \pm 0.7$ & $-1.73 \pm 0.33$ & $0.28 \pm 0.30$ \\
\hline B324 & 2 & $1.0 \pm 0.1$ & $-0.48 \pm 0.02$ & $-0.11 \pm 0.11$ \\
\hline B327 & 2 & $5.4 \pm 1.4$ & $-1.69 \pm 0.31$ & $0.30 \pm 0.16$ \\
\hline B328 & 2 & $11.0 \pm 2.4$ & $-1.71 \pm 0.30$ & $0.62 \pm 0.32$ \\
\hline B337 & 2 & $4.9 \pm 2.9$ & $-0.59 \pm 0.05$ & $0.00 \pm 0.11$ \\
\hline B347 & 2 & $10.2 \pm 2.4$ & $-1.97 \pm 0.14$ & $0.68 \pm 0.36$ \\
\hline B350 & 2 & $9.3 \pm 2.3$ & $-1.45 \pm 0.26$ & $0.38 \pm 0.28$ \\
\hline B365 & 2 & $6.6 \pm 3.1$ & $-0.95 \pm 0.13$ & $0.08 \pm 0.16$ \\
\hline B380 & 2 & $1.0 \pm 0.1$ & $-1.51 \pm 0.03$ & $-0.19 \pm 0.13$ \\
\hline B383 & 2 & $11.3 \pm 2.4$ & $-0.33 \pm 0.06$ & $0.28 \pm 0.05$ \\
\hline B393 & 2 & $11.2 \pm 1.4$ & $-0.63 \pm 0.05$ & $-0.03 \pm 0.14$ \\
\hline B398 & 2 & $15.0 \pm 2.4$ & $-0.32 \pm 0.06$ & $0.23 \pm 0.09$ \\
\hline B401 & 2 & $10.2 \pm 2.4$ & $-1.94 \pm 0.14$ & $0.47 \pm 0.31$ \\
\hline NB16 & 2 & $7.2 \pm 3.5$ & $-0.86 \pm 0.11$ & $0.27 \pm 0.11$ \\
\hline NB67 & 2 & $10.1 \pm 3.2$ & $-0.98 \pm 0.18$ & $0.38 \pm 0.13$ \\
\hline NB68 & 2 & $7.6 \pm 2.3$ & $-0.28 \pm 0.06$ & $0.19 \pm 0.07$ \\
\hline NB74 & 2 & $15.0 \pm 1.6$ & $-0.04 \pm 0.06$ & $0.56 \pm 0.13$ \\
\hline NB81 & 2 & $4.5 \pm 1.1$ & $-0.19 \pm 0.06$ & $0.08 \pm 0.06$ \\
\hline NB83 & 2 & $7.3 \pm 3.6$ & $-0.68 \pm 0.09$ & $0.05 \pm 0.13$ \\
\hline NB87 & 2 & $8.8 \pm 2.4$ & $0.48 \pm 0.08$ & $0.09 \pm 0.04$ \\
\hline NB89 & 2 & $6.8 \pm 1.7$ & $-0.41 \pm 0.04$ & $0.18 \pm 0.07$ \\
\hline NB91 & 2 & $7.8 \pm 2.4$ & $-0.28 \pm 0.06$ & $0.13 \pm 0.07$ \\
\hline B240 & 3 & $11.0 \pm 1.1$ & $-1.82 \pm 0.11$ & $0.06 \pm 0.24$ \\
\hline B058 & 3 & $6.4 \pm 4.1$ & $-0.57 \pm 0.10$ & $-0.34 \pm 0.15$ \\
\hline B178 & 3 & $6.3 \pm 1.8$ & $-0.61 \pm 0.06$ & $0.38 \pm 0.09$ \\
\hline G001 & 3 & $11.4 \pm 1.7$ & $-0.63 \pm 0.06$ & $0.42 \pm 0.05$ \\
\hline B358 & 3 & $10.4 \pm 1.9$ & $-2.00 \pm 0.11$ & $-0.00 \pm 0.27$ \\
\hline
\end{tabular}

${ }^{a}$ Dataset label: 1 - this work; 2 - Beasley et al. (2004); 3 - Worthey et al. (1994); and Kuntschner et al. (2002). 